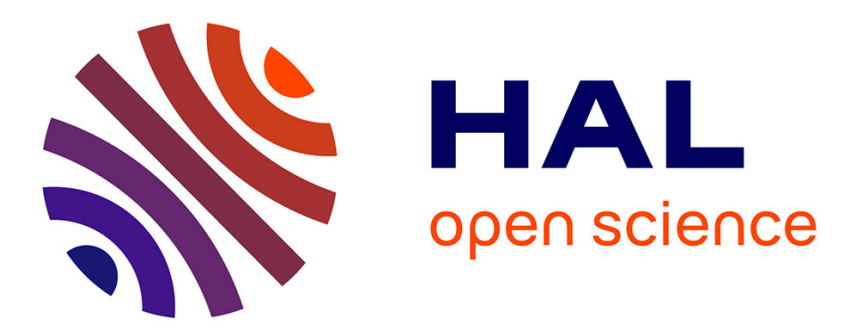

\title{
DIFFERENTIAL SYMMETRY BREAKING OPERATORS. I. GENERAL THEORY AND F-METHOD
}

Toshiyuki Kobayashi, Michael Pevzner

\section{- To cite this version:}

Toshiyuki Kobayashi, Michael Pevzner. DIFFERENTIAL SYMMETRY BREAKING OPERATORS.

I. GENERAL THEORY AND F-METHOD. Selecta Mathematica (New Series), 2016, 22 (2), pp.801

- 845. 10.1007/s00029-015-0207-9 . hal-01907210

\section{HAL Id: hal-01907210 \\ https://hal.science/hal-01907210}

Submitted on 28 Oct 2018

HAL is a multi-disciplinary open access archive for the deposit and dissemination of scientific research documents, whether they are published or not. The documents may come from teaching and research institutions in France or abroad, or from public or private research centers.
L'archive ouverte pluridisciplinaire HAL, est destinée au dépôt et à la diffusion de documents scientifiques de niveau recherche, publiés ou non, émanant des établissements d'enseignement et de recherche français ou étrangers, des laboratoires publics ou privés. 


\title{
DIFFERENTIAL SYMMETRY BREAKING OPERATORS. I. GENERAL THEORY AND F-METHOD.
}

\author{
TOSHIYUKI KOBAYASHI, MICHAEL PEVZNER
}

\begin{abstract}
We prove a one-to-one correspondence between differential symmetry breaking operators for equivariant vector bundles over two homogeneous spaces and certain homomorphisms for representations of two Lie algebras, in connection with branching problems of the restriction of representations.

We develop a new method (F-method) based on the algebraic Fourier transform for generalized Verma modules, which characterizes differential symmetry breaking operators by means of certain systems of partial differential equations.

In contrast to the setting of real flag varieties, continuous symmetry breaking operators of Hermitian symmetric spaces are proved to be differential operators in the holomorphic setting. In this case symmetry breaking operators are characterized by differential equations of second order via the F-method.
\end{abstract}

Key words and phrases: branching laws, F-method, symmetric pair, invariant theory, Verma modules, Hermitian symmetric spaces.

\section{CONTENTS}

1. Introduction 2

2. Differential intertwining operators 4

2.1. Differential operators between two manifolds 4

2.2. Induced modules 7

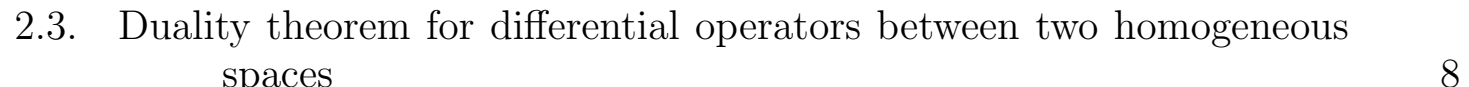

2.4. Construction of $D_{X_{b y}}$.

2.5. Proof of Theorem $[2.9]^{\text {s. }}$

3. Algebraic Fourier transform for generalized Verma modules 17

3.1. Weyl algebra and algebraic Fourier transform 17

3.2. Holomorphic vector fields associated to the Gelfand-Naimark decomposition 20

3.3. Fourier transform of principal series representations 22

\begin{tabular}{lll}
\hline 3.4. & Fourier transform on the real flag varieties & 25
\end{tabular}

Date: June 13, 2017.

2010 Mathematics Subject Classification. Primary 22E47; Secondary 22E46, 11F55, 53C10. 


\begin{tabular}{|c|c|}
\hline \multicolumn{2}{|l|}{$\begin{array}{l}\text { 4. F-method } \\
\text { 4.1. Construction of equivariant differential operators by algebraic Fourier }\end{array}$} \\
\hline transform & 28 \\
\hline 4.2. Symbol map and reverșing signatures & 30 \\
\hline 4.3. Proof of Theorem $4.1(2)$ & 31 \\
\hline 4.4. Recipe of the F-method for abelian nilradical $\mathfrak{n}_{+}$ & 31 \\
\hline 4.5. $\quad$ F-method - supplement for vector valued cases. & 34 \\
\hline 5. Localness and extension theorem for symmetry breaking operators & 36 \\
\hline 5.1. Formulation of the localness theorem & 36 \\
\hline 5.2. Proof of the localness theorem & 39 \\
\hline 5.3. Automatic continuity theorem in the unitary case & 41 \\
\hline 5.4. Orthogonal projectors & 43 \\
\hline Acknowledgements & 43 \\
\hline References & 4 \\
\hline
\end{tabular}

\section{INTRODUCTION}

Let $\mathcal{W} \rightarrow Y$ and $\mathcal{V} \rightarrow X$ be two vector bundles with a smooth map $p: Y \rightarrow X$. Then we can define "differential operators" $D: C^{\infty}(X, \mathcal{V}) \rightarrow C^{\infty}(Y, \mathcal{W})$ between the spaces of smooth sections (Definition 2.11).

Suppose that $G^{\prime} \subset G$ is a pair of Lie groups acting equivariantly on $\mathcal{W} \rightarrow Y$ and $\mathcal{V} \rightarrow X$, respectively, and that $p$ is $G^{\prime}$-equivariant. The object of the present work is the study of $G^{\prime}$-intertwining differential operators (differential symmetry breaking operators). If $\mathcal{W}$ is isomorphic to the pull-back $p^{*} \mathcal{V}$, then the restriction map $\left.f \mapsto f\right|_{Y}$ is obviously a $G^{\prime}$-intertwining operator (and a differential operator of order zero). In the general setting where there is no morphism from $p^{*} \mathcal{V}$ to $\mathcal{W}$, non-zero $G^{\prime}$-intertwining differential operators may or may not exist.

Suppose that $G$ acts transitively on $X$ and $G^{\prime}$ acts transitively on $Y$. We write $X$ (respectively $Y$ ) as a homogeneous space $=G / H$ (respectively $Y=G^{\prime} / H^{\prime}$ ). The first main result is a duality theorem that gives a one-to-one correspondence between $G^{\prime}$-intertwining differential operators and $\left(\mathfrak{g}^{\prime} \cdot H^{\prime}{ }_{9}\right.$-homomorphisms for induced representations of Lie algebras (see Corollary 2.10 for the precise notation):

thm:thma Theorem A. Suppose $H^{\prime} \subset H$. Then there is a natural bijection:

eqn : ThmA

$$
D_{X \rightarrow Y}: \operatorname{Hom}_{\left(\mathfrak{g}^{\prime}, H^{\prime}\right)}\left(\operatorname{ind}_{\mathfrak{h}^{\prime}}^{\mathfrak{g}^{\prime}}\left(W^{\vee}\right), \operatorname{ind}_{\mathfrak{h}}^{\mathfrak{g}}\left(V^{\vee}\right)\right) \stackrel{\sim}{\longrightarrow} \operatorname{Diff}_{G^{\prime}}\left(\mathcal{V}_{X}, \mathcal{W}_{Y}\right)
$$

This generalizes a well-known result in the case where $G$ and $G_{H .}^{\prime}$ are the same reductive group and where $X$ and $Y$ are the same flag variety ([Kos74, HJ82]). 
By a branching problem we mean a problem of understanding how a given representation of a group $G$ behaves when restricted to a subgroup $G^{\prime}$. For a unitary representation $\pi$ of $G$, branching problems concern a decomposition of $\pi$ into the direct integral of irreducible unitary representations of $G^{\prime}$ (branching law).

More generally, for non-unitary representations $\pi$ and $\tau$ of $G$ and $G^{\prime}$, respectively, we may consider the space $\operatorname{Hom}_{G^{\prime}}\left(\left.\pi\right|_{G^{\prime}}, \tau\right)$ of continuous $G^{\prime}$-homomorphisms. The right-hand side of (1.1) concerns branching problems with respect to the restriction from $G$ to $G^{\prime}$, whereas the left-hand side of (1.1) concerns branching laws of "generalized Verma modules".

If $\operatorname{Diff}_{G^{\prime}}\left(\mathcal{V}_{X}, \mathcal{W}_{Y}\right)$ in $(1.1)$ is one-dimensional, we may regard its generator as canonical up to a scalar and be tempted to find an explicit description for such a natural differential symmetry breaking operator. It should be noted that seeking explicit formulæ of intertwining operators is much more involved than finding abstract branching laws, as we may observe with the celebrated Rankin-Cohen brackets which appear as symmetry breaking operators in the decomposition of the tensor product of two holomorphic discrete series representations of $S L(2, \mathbb{R})$ (see [DPO7, KP15-2] for a detailed discussion).

The condition $\operatorname{dim} \operatorname{Diff}_{G^{\prime}}\left(\mathcal{V}_{X}, \mathcal{W}_{Y}\right) \leq 1$ is iften $_{1}$ fulfilled when $\mathfrak{h}$ is a parabolic subalgebra of $\mathfrak{g}$ with abelian nilradical, (see [K14, Theorem 2.7]). Moreover, finding all bundles $\mathcal{W}_{Y}$ for which such nontrivial intertwining operators exist is aR.part of the initial problem, which reduces to abstract branching problems (see [KP15-2, Fact t?act:

We propose a new method to find explicit expressions for differential symmetry breaking operators appearing in this geometric setting. We call it the F-method, where $\mathrm{F}$ stands for the Fourier transform. More precisely, we consider an "algebraic Fourier transform" of generalized Verma modules, and characterize symmetry breaking operators by means of certain systems of partial differential equations. If $\mathfrak{h}$ is a parabolic subalgebra with abelian nilradical, then the system is of second order although the resulting differential symmetry breaking operators may be of any higher order. The characterization is performed by applying an algebraic Fourier transform 44 (see Definition 3.1]. ${ }^{4}$ detailed recipe of the $F$-method is described in Section 4.4 relying on Theorem 4.1 and Proposition 3.11 .

In general, symmetry breaking operators between two principal series representations of real reductive Lie groups $G^{\prime} \subset G$ are given by integro-differential operators in

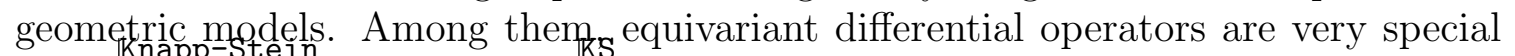
(e.g. [KnSt71] for $G^{\prime}=G$ and [KS14] for $G^{\prime} \varsubsetneqq G$ ). However, in the case where $X$ is a Hermitian symmetric space, $Y$ a subsymmetric space, $G^{\prime} \subset G$ are the groups of biholomorphic transformations of $Y \hookrightarrow X$, respectively, we prove the following localness and extension theorem: 
thm:intro Theorem B. Any continuous $G^{\prime}$-homomorphism from $\mathcal{O}(X, \mathcal{V})$ to $\mathcal{O}(Y, \mathcal{W})$ is given by a holomorphic differential operator, which extends to the whole flag variety.

See Theorem $\frac{t h m: C}{5.3}$ for the precise statement. Theorem $\mathbb{B}$ includes the case of the tensor product of two holomorphic discrete series representations corresponding to the setting where $G \simeq G^{\prime} \times G^{\prime}$ and $X$ AT $\sim 2 \times Y$ as a special case.

In the second part of the work [KPI5-2] we apply the F-method to Hermitian symmetric spaces to find explicit formulæ of differential symmetry breaking operators in the six parabolic geometries arising from symmetric pairs of split rank one.

The authors are grateful to the referee for enlightening remarks and for suggesting to divide the original manuscript (old title "Rankin-Cohen operators for symmetric pairs") into two parts and to write more detailed proofs and explanations in the first part, not only for specialists but also for a broader audience. We would like to extend a special thanks to Dr. T. Kubo for providing valuable and constructive suggestions in respect to its legibility.

Notation: $\mathbb{N}=\{0,1,2, \cdots\}$.

sec : 2 part 1

\section{Differential intertwining operators}

In this section we discuss equivariant differential operators between sections of homogeneous vector bundles in a more general setting than the usual. Namely, we consider vector bundles admitting a morphism between their base spaces. In this generality, we establish a natural bijection between such differential operators (differential symmetry breaking operators) and certain homomorphisms arising from the branching problems for infinite-dimensional representations of Lie algebras, see Theorem 2.9. (duality theorem).

$\sec : 21$

2.1. Differential operators between two manifolds. We understand the notion of differential operators between two vector bundles in the usual sense when the bundles are defined over the same base space. We extend this terminology in a more general setting, where there exists a morphism between base spaces. Let $\mathcal{V} \rightarrow X$ be a vector bundle over a smooth manifold $X$. We write $C^{\infty}(X, \mathcal{V})$ for the space of smooth sections, which is endowed with the Fréchet topology of uniform convergence of sections and their derivatives of finite order on compact sets. Let $\mathcal{W} \rightarrow Y$ be another vector bundle, and $p: Y \rightarrow X$ a smooth map between the base spaces.

def:21 Definition 2.1. We say that a continuous linear map $T: C^{\infty}(X, \mathcal{V}) \rightarrow C^{\infty}(Y, \mathcal{W})$ is a differential operator if $T$ satisfies

eqn: suppT

$$
p(\operatorname{Supp} T f) \subset \operatorname{Supp} f \quad \text { for any } f \in C^{\infty}(X, \mathcal{V}) .
$$


We write $\operatorname{Diff}\left(\mathcal{V}_{X}, \mathcal{W}_{Y}\right)$ for the vector space of differential operators from $C^{\infty}(X, \mathcal{V})$ to $C^{\infty}(Y, \mathcal{W})$.

The condition 2.10 supp $\frac{\text { show }}{2.1}$ shat $T$ is a local operator in the sense that for any open subset $U$ of $X, T$ induces a continuous linear map:

$$
T_{U}: C^{\infty}\left(U,\left.\mathcal{V}\right|_{U}\right) \longrightarrow C^{\infty}\left(p^{-1}(U),\left.\mathcal{W}\right|_{p^{-1}(U)}\right)
$$

Remark 2.2. If $X=Y$ and $p$ is the identity map, then the condition (2.1) is equivalent to $T$ being a differential operator in the uspual sense owing to Peetre's celebrated theorem [Pee59. Our proof of Lemma 2.3 in this special case gives an account of this classical theorem by using the theory of distributions due to L. Schwartz [\$66].

Let $\Omega_{X}:=\left|\Lambda^{\text {top }} T^{\vee}(X)\right|$ be the bundle of densities. For a vector bundle $\mathcal{V} \rightarrow X$, we set $\mathcal{V}^{\vee}:=\bigsqcup_{x \in X} \mathcal{V}_{x}^{\vee}$ where $\mathcal{V}_{x}^{\vee}:=\operatorname{Hom}_{\mathbb{C}}\left(\mathcal{V}_{x}, \mathbb{C}\right)$, and denote by $\mathcal{V}^{*}$ the dualizing bundle $\mathcal{V}^{\vee} \otimes \Omega_{X}$. In what follows $\mathcal{D}^{\prime}\left(X, \mathcal{V}^{*}\right)$ (respectively, $\mathcal{E}^{\prime}\left(X, \mathcal{V}^{*}\right)$ ) denotes the space of $\mathcal{V}^{*}$-valued distributions (respectively, those with compact support). We shall regard distributions as generalized functions à la Gelfand rather than continuous linear forms on $C_{c}^{\infty}(X)$ or $C^{\infty}(X)$. In particular, we sometimes write as

$$
\mathcal{E}^{\prime}\left(X, \Omega_{X}\right) \rightarrow \mathbb{C}, \quad \omega \mapsto \int_{X} \omega,
$$

to denote the natural pairing $\left\langle\omega, \mathbf{1}_{X}\right\rangle$ of $\omega$ with the constant function $\mathbf{1}_{X}$ on $X$. Composing (2.2) with the contraction on the fiber, we get a natural bilinear map

$$
C^{\infty}(X, \mathcal{V}) \times \mathcal{E}^{\prime}\left(X, \mathcal{V}^{*}\right) \rightarrow \mathbb{C}, \quad(f, \omega) \mapsto\langle f, \omega\rangle=\int_{X} f \omega
$$

Let $\mathcal{V}^{*} \otimes \mathcal{W}$ denote the tensor product bundle over $X \times Y$ of the two vector bundles $\mathcal{V}^{*} \rightarrow X$ and $\mathcal{W} \rightarrow Y$. Then for any continuous linear map $T: C^{\infty}(X, \mathcal{V}) \rightarrow C^{\infty}(Y, \mathcal{W})$ there exists a unique distribution $K_{T} \in \mathcal{D}^{\prime}\left(X \times Y, \mathcal{V}^{*} \otimes \mathcal{W}\right)$ such that the projection on the second factor $\operatorname{pr}_{2}: X \times Y \rightarrow Y$ is proper on the support of $K_{T}$ and such that

$$
(T f)(y)=\left\langle K_{T}(\cdot, y), f(\cdot)\right\rangle \text { for any } f \in C^{\infty}(X, \mathcal{V}),
$$

by the Schwartz kernel theorem.

Given a map $p: Y \rightarrow X$, we set

$$
\Delta(Y):=\{(p(y), y): y \in Y\} \subset X \times Y .
$$

The following lemma characterizes differential operators by means of the distribution kernels $K_{T}$.

lem:1 Lemma 2.3. Let $p: Y \rightarrow X$ be a smooth map. A continuous operator $T_{2}: C^{\infty}(X, \mathcal{V}) \rightarrow$ $C^{\infty}(Y, \mathcal{W})$ is a differential operator in the sense of Definition 2.1 if and only if $\operatorname{Supp} K_{T} \subset \Delta(Y)$. 
Proof. Suppose $\operatorname{Supp} K_{T} \subset \Delta(Y)$. Let $\left(x_{o}, y_{o}\right) \in \Delta(Y)$ and take a neighborhood $U$ of $x_{o}=p\left(y_{o}\right)$ in $X$ and a neighborhood $U^{\prime}$ of $y_{o}$ in $Y$ such that $U^{\prime} \subset p^{-1}(U)$. We trivialize the bundles locally as $\left.\mathcal{V}\right|_{U} \simeq U \times V$ and $\left.\mathcal{W}\right|_{U^{\prime}} \simeq U^{\prime} \times W$. Let $\left(x_{1}, \cdots, x_{m}\right)$ be the coordinates in $U$. According to the structural theory of distributions supported on a submanifold $\Delta Y \subset X \times Y$ [5666, Chapter III, Théorème XXXVII], there exists a unique family $h_{\alpha}(y) \in \mathcal{D}^{\prime}\left(U^{\prime}\right) \otimes W$ for a finite number of multi-indices $\alpha=\left(\alpha_{1}, \cdots, \alpha_{m}\right) \in \mathbb{N}^{m}$, such that $\left\langle K_{T}, f\right\rangle \in \mathcal{D}^{\prime}\left(U^{\prime}\right) \otimes \operatorname{Hom}_{\mathbb{C}}(V, W)$ is locally given as a finite sum

eqn: halpha

$$
\sum_{\alpha} h_{\alpha}(y) \frac{\partial^{|\alpha|} f}{\partial x^{\alpha}}(p(y))
$$

for every $f \in C^{\infty}(X, \mathcal{V})$. Hence $\left\langle\left. K_{d f_{f}} f{ }_{21}\right|_{U^{\prime}}=0\right.$ if $\left.f\right|_{U}=0$. Thus $T$ is a differential operator in the sense of Definition 2.1.

Conversely, take any $\left(x_{o}, y_{o}\right) \in \operatorname{Supp} K_{T}$. By the definition of the distribution kernel $K_{T}$, for any neighborhood $S$ of $x_{o}$ in $X$ there exists $f \in C^{\infty}(X, \mathcal{V})$ such that Supp $f \subset S$ and $\left(x_{o}, y_{o}\right) \in \operatorname{Supp} f \times \operatorname{Supp} T f$. If $T$ is a differential operator then by (2.1)

$$
p(\operatorname{Supp} T f) \subset \operatorname{Supp} f \subset S .
$$

Since $S$ is an arbitrary neighborhood of $x_{o}, p\left(y_{o}\right)$ must coincide with $x_{o}$. Hence Supp $K_{T} \subset \Delta(Y)$.

By $\frac{\text { ang }}{2.4}$, halpha , the terminology "differential operators" in Definition 2.1 is justified as follows:

Example 2.4. (1) Let $p: Y \rightarrow X$ be a submersion. Choose an atlas of local coordinates $\left\{\left(x_{i}, z_{j}\right)\right\}$ on $Y$ in such a way that $\left\{x_{i}\right\}$ form an atlas on $X$. Then, every $T \in \operatorname{Diff}\left(\mathcal{V}_{X}, \mathcal{W}_{Y}\right)$ is locally of the form

$$
\sum_{\alpha \in \mathbb{N}^{d i m} X} h_{\alpha}(x, z) \frac{\partial^{|\alpha|}}{\partial x^{\alpha}} \quad \text { (finite sum), }
$$

where $h_{\alpha}(x, z)$ are $\operatorname{Hom}(V, W)$-valued smooth functions on $Y$.

(2) Let $i: Y \hookrightarrow X$ be an immersion. Choose an atlas of local coordinates $\left\{\left(y_{i}, z_{j}\right)\right\}$ on $X$ in such a way that $\left\{y_{i}\right\}$ form an atlas on $Y$. Then, every $T \in \operatorname{Diff}\left(\mathcal{V}_{X}, \mathcal{W}_{Y}\right)$ is locally of the form

$$
\sum_{(\alpha, \beta) \in \mathbb{N} \operatorname{dim} X} g_{\alpha \beta}(y) \frac{\partial^{|\alpha|+|\beta|}}{\partial y^{\alpha} \partial z^{\beta}} \quad \text { (finite sum), }
$$

where $g_{\alpha, \beta}(y)$ are $\operatorname{Hom}(V, W)$-valued smooth functions on $Y$.

Next, suppose that the two vector bundles $\mathcal{V} \rightarrow X$ and $\mathcal{W} \rightarrow Y$ are equivariant with respect to a given Lie group $G$. Then we have natural actions of $G$ on the Fréchet 
spaces $C^{\infty}(X, \mathcal{V})$ and $C^{\infty}(Y, \mathcal{W})$ by translations. Denote by $\operatorname{Hom}_{G}\left(C^{\infty}(X, \mathcal{V}), C^{\infty}(Y, \mathcal{W})\right)$ the space of continuous $G$-homomorphisms. We set

$$
\operatorname{Diff}_{G}\left(\mathcal{V}_{X}, \mathcal{W}_{Y}\right):=\operatorname{Diff}\left(\mathcal{V}_{X}, \mathcal{W}_{Y}\right) \cap \operatorname{Hom}_{G}\left(C^{\infty}(X, \mathcal{V}), C^{\infty}(Y, \mathcal{W})\right)
$$

Example 2.5. Suppose $X$ and $Y$ are both Euclidean vector spaces with an injective linear map $p: Y \hookrightarrow X$. If $G$ contains the subgroup of all translations of $Y$ then $\operatorname{Diff}_{G}\left(\mathcal{V}_{X}, \mathcal{W}_{Y}\right)$ is a subspace of the space of differential operators with constant coefficients.

An analogous notion can be defined in the holomorphic setting. Let $\mathcal{V} \rightarrow X$ and $\mathcal{W} \rightarrow Y$ be two holomorphic vector bundles with a holomorphic map $p: Y \rightarrow X$ between the complex base manifolds $X$ and $Y$. We say a differential operator $T$ : $C^{\infty}(X, \mathcal{V}) \rightarrow C^{\infty}(Y, \mathcal{W})$ is holomorphic if

$$
T_{U}\left(\mathcal{O}\left(U,\left.\mathcal{V}\right|_{U}\right)\right) \subset \mathcal{O}\left(p^{-1}(U),\left.\mathcal{W}\right|_{p^{-1}(U)}\right)
$$

for any open subset $U$ of $X$. We denote by $\operatorname{Diff}^{\text {hol }}\left(\mathcal{V}_{X}, \mathcal{W}_{Y}\right)$ the vector space of holomorphic differential operators. When a Lie group $G$ acts biholomorphically on the two holomorphic vector bundles $\mathcal{V} \rightarrow X$ and $\mathcal{W} \rightarrow Y$, we set

$$
\operatorname{Diff}_{G}^{\text {hol }}\left(\mathcal{V}_{X}, \mathcal{W}_{Y}\right):=\operatorname{Diff}^{\text {hol }}\left(\mathcal{V}_{X}, \mathcal{W}_{Y}\right) \cap \operatorname{Hom}_{G}\left(C^{\infty}(X, \mathcal{V}), C^{\infty}(Y, \mathcal{W})\right)
$$

2.2. Induced modules. Let $\mathfrak{g}$ be a Lie algebra over $\mathbb{C}$, and $U(\mathfrak{g})$ its universal enveloping algebra. Let $\mathfrak{h}$ be a Lie subalgebra of $\mathfrak{g}$.

Definition 2.6. For an $\mathfrak{h}$-module $V$, we define the induced $U(\mathfrak{g})$-module $\operatorname{ind}_{\mathfrak{h}}^{\mathfrak{g}}(V)$ as

$$
\operatorname{ind}_{\mathfrak{h}}^{\mathfrak{g}}(V):=U(\mathfrak{g}) \otimes_{U(\mathfrak{h})} V .
$$

If $\mathfrak{h}$ is a Borel subalgebra and $\operatorname{dim} V=1$, then the $\mathfrak{g}$-module $\operatorname{ind}_{\mathfrak{h}} \mathfrak{g}(V)$ is the Verma module.

For later purposes we formulate the following statement in terms of the contragredient representation $V^{\vee}$. Let $\mathfrak{h}^{\prime}$ be another Lie subalgebra of $\mathfrak{g}$.

prop:35 Proposition 2.7. For a finite-dimensional $\mathfrak{h}^{\prime}$-module $W$ we have:

(1) $\operatorname{Hom}_{\mathfrak{g}}\left(\operatorname{ind}_{\mathfrak{h}^{\prime}}^{\mathfrak{g}}\left(W^{\vee}\right), \operatorname{ind}_{\mathfrak{h}}^{\mathfrak{g}}\left(V^{\vee}\right)\right) \simeq \operatorname{Hom}_{\mathfrak{h}^{\prime}}\left(W^{\vee}, \operatorname{ind}_{\mathfrak{h}}^{\mathfrak{g}}\left(V^{\vee}\right)\right)$.

(2) If $\mathfrak{h}^{\prime} \not \subset \mathfrak{h}$, then $\operatorname{Hom}_{\mathfrak{h}^{\prime}}\left(W^{\vee}, \operatorname{ind}_{\mathfrak{h}}^{\mathfrak{g}}\left(V^{\vee}\right)\right)=\{0\}$.

Proof. The first statement is due to the functoriality of the tensor product.

For the second statement it suffices to treat the case where $\mathfrak{h}^{\prime}$ is one-dimensional. Then the assumption $\mathfrak{h}^{\prime} \not \subset \mathfrak{h}$ implies that $\mathfrak{h}^{\prime} \cap \mathfrak{h}=\{0\}$, and therefore there is a direct sum decomposition of vector spaces:

$$
\mathfrak{g}=\mathfrak{h}^{\prime}+\mathfrak{q}+\mathfrak{h},
$$


for some subspace $\mathfrak{q}$ in $\mathfrak{g}$. We fix a basis $X_{1}, \cdots, X_{n}$ of $\mathfrak{q}$, and define a subspace of $U(\mathfrak{g})$ by

$$
U^{\prime}(\mathfrak{q}):=\mathbb{C}-\operatorname{span}\left\{X_{1}^{\alpha_{1}} \cdots X_{n}^{\alpha_{n}}:\left(\alpha_{1}, \cdots, \alpha_{n}\right) \in \mathbb{N}^{n}\right\} .
$$

Then, by the Poincaré-Birkhoff-Witt theorem we have an isomorphism of 'h'-modules:

$$
\operatorname{ind}_{\mathfrak{h}}^{\mathfrak{g}}\left(V^{\vee}\right) \simeq U\left(\mathfrak{h}^{\prime}\right) \otimes_{\mathbb{C}} U^{\prime}(\mathfrak{q}) \otimes_{\mathbb{C}} V^{\vee} .
$$

In particular, $\operatorname{ind}_{\mathfrak{h}}^{\mathfrak{g}}\left(V^{\vee}\right)$ is a free $U\left(\mathfrak{h}^{\prime}\right)$-module. Hence there does not exist a non-zero finite-dimensional $\mathfrak{h}^{\prime}$-submodule in the $\mathfrak{g}$-module $\operatorname{ind}_{\mathfrak{h}}^{\mathfrak{g}}\left(V^{\vee}\right)$.

rem:231 Remark 2.8. We shall see in Theorem 2.9: that dim $\mathbb{C}_{\mathbb{C}} \operatorname{Hom}_{\mathfrak{g}^{\prime}}\left(\operatorname{ind}_{\mathfrak{h}^{\prime}}^{\mathfrak{g}^{\prime}}\left(W^{\vee}\right), \operatorname{ind}_{\mathfrak{h}}^{\mathfrak{g}}\left(V^{\vee}\right)\right)$ is equal to the dimension of the space of differential symmetry breaking operators from $C^{\infty}(X, \mathcal{V})$ to $C^{\infty}(Y, \mathcal{W})$ when $H^{\prime}$ is connected. In [KP15-2, Section ??], we give a family of sextuples $\left(\mathfrak{g}, \mathfrak{g}^{\prime}, \mathfrak{h}, \mathfrak{h}^{\prime}, V, W\right)$ such that this dimension is one.

sec : 33

2.3. Duality theorem for differential operators between two homogeneous spaces. Let $G$ be a real Lie group, and $\mathfrak{g}(\mathbb{R}):=\operatorname{Lie}(G)$. We denote by $U(\mathfrak{g})$ the universal enveloping algebra of the complexified Lie algebra $\mathfrak{g}:=\mathfrak{g}(\mathbb{R}) \otimes_{\mathbb{R}} \mathbb{C}$. Analogous notations will be applied to other Lie groups.

Let $H$ be a closed subgroup of $G$. Given a finite-dimensional representation $\lambda$ : $H \rightarrow G L_{\mathbb{C}}(V)$ we define the homogeneous vector bundle $\mathcal{V}_{X} \equiv \mathcal{V}:=G \times_{H} V$ over $X:=G / H$. As a $G$-module, the space $C^{\infty}(X, \mathcal{V})$ of smooth sections is identified with the following subspace of $C^{\infty}(G, V) \simeq C^{\infty}(G) \otimes V$ :

$$
\begin{aligned}
C^{\infty}(G, V)^{H} & :=\left\{f \in C^{\infty}(G, V): f(g h)=\lambda(h)^{-1} f(g) \text { for any } g \in G, h \in H\right\} \\
& \simeq\left\{F \in C^{\infty}(G) \otimes V: \lambda(h) F(g h)=F(g) \text { for any } g \in G, h \in H\right\} .
\end{aligned}
$$

In dealing with a representation $V$ of a disconnected subgroup $H$ (e.g. $H$ is a parabolic subgroup of a real reductive Lie group $G$ ), we notice that the diagonal $H$-action on $U(\mathfrak{g}) \otimes_{\mathbb{C}} V^{\vee}$ defines a representation of $H$ on $\operatorname{ind}_{\mathfrak{h}}^{\mathfrak{g}}\left(V^{\vee}\right)=U(\mathfrak{g}) \otimes_{\mathfrak{h}} V^{\vee}$ and thus ind $\operatorname{ig}_{\mathfrak{h}}\left(V^{\vee}\right)$ is endowed with a $(\mathfrak{g}, H)$-module structure.

thm:surject Theorem 2.9 (Duality theorem). Let $H^{\prime} \subset H$ be (possibly disconnected) closed subgroups of a Lie group $G$ with Lie algebras $\mathfrak{h}^{\prime} \subset \mathfrak{h}$, respectively. Suppose $V$ and $W$ are finite-dimensional representations of $H$ and $H^{\prime}$, respectively. Let $G^{\prime}$ be any subgroup of $G$ containing $H^{\prime}$, and $\mathcal{V}_{X}:=G \times_{H} V$ and $\mathcal{W}_{Y}:=G^{\prime} \times_{H^{\prime}} W$ be the corresponding homogeneous vector bundles. Then, there is a natural linear isomorphism:

eqn:DXYH (2.6)

$$
D_{X \rightarrow Y}: \operatorname{Hom}_{H^{\prime}}\left(W^{\vee}, \operatorname{ind}_{\mathfrak{h}}^{\mathfrak{g}}\left(V^{\vee}\right)\right) \stackrel{\sim}{\longrightarrow} \operatorname{Diff}_{G^{\prime}}\left(\mathcal{V}_{X}, \mathcal{W}_{Y}\right),
$$

or equivalently,

$$
D_{X \rightarrow Y}: \operatorname{Hom}_{\left(\mathfrak{g}^{\prime}, H^{\prime}\right)}\left(\operatorname{ind}_{\mathfrak{h}^{\prime}}^{\mathfrak{g}^{\prime}}\left(W^{\vee}\right), \operatorname{ind}_{\mathfrak{h}}^{\mathfrak{g}}\left(V^{\vee}\right)\right) \stackrel{\sim}{\longrightarrow} \operatorname{Diff}_{G^{\prime}}\left(\mathcal{V}_{X}, \mathcal{W}_{Y}\right) .
$$


For $\varphi \in \operatorname{Hom}_{H^{\prime}}\left(W^{\vee}, \operatorname{ind}_{\mathfrak{h}}^{\mathfrak{g}}\left(V^{\vee}\right)\right)$ and $F \in C^{\infty}(X, \mathcal{V}) \simeq C^{\infty}(G, V)^{H}, D_{X \rightarrow Y}(\varphi) F \in$ $C^{\infty}(Y, \mathcal{W}) \simeq C^{\infty}\left(G^{\prime}, W\right)^{H^{\prime}}$ is given by the following formula:

eqn:Dxy

$$
\left\langle D_{X \rightarrow Y}(\varphi) F, w^{\vee}\right\rangle=\left.\sum_{j}\left\langle d R\left(u_{j}\right) F, v_{j}^{\vee}\right\rangle\right|_{G^{\prime}} \quad \text { for } w^{\vee} \in W^{\vee},
$$

where $\varphi\left(w^{\vee}\right)=\sum_{j} u_{j} v_{j}^{\vee} \in \operatorname{ind}_{\mathfrak{h}}^{\mathfrak{g}}\left(V^{\vee}\right)\left(u_{j} \in U(\mathfrak{g}), v_{j}^{\vee} \in V^{\vee}\right)$.

When $H^{\prime}$ is connected, we can write the left-hand side of (2.0) by means of Lie algebras.

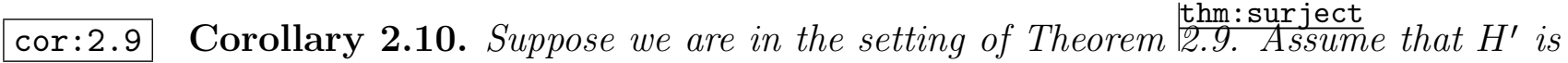
connected. Then there is a natural linear isomorphism:

$$
D_{X \rightarrow Y}: \operatorname{Hom}_{\mathfrak{h}^{\prime}}\left(W^{\vee}, \operatorname{ind}_{\mathfrak{h}}^{\mathfrak{g}}\left(V^{\vee}\right)\right) \stackrel{\sim}{\longrightarrow} \operatorname{Diff}_{G^{\prime}}\left(\mathcal{V}_{X}, \mathcal{W}_{Y}\right),
$$

or equivalently,

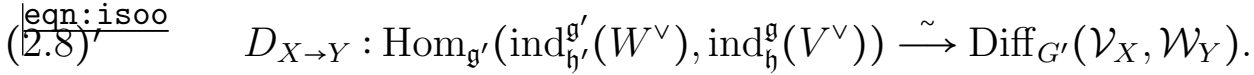

The construction of $D_{\text {subsec:DXY }}$ and the fact that the formula 2.7 is well-defined will be explained in Section 2.4 .

Remark 2.11. (1) Corollary 2.10 is known when $X=Y$, i.e. $G_{\mathrm{K} / \overline{7}}^{\prime} G$ and $H^{\prime}=H$, especially in the setting of complex flag varieties, see e.g. [Kos74, $\mathrm{HJ} 82$.

(2) When $\mathfrak{g}^{\prime}$ is a reductive subalgebra and $\mathfrak{h}^{\prime}$ is a parabolic subalgebra, the existence of an $\mathfrak{h}^{\prime}$-module $W$ for which the left-hand side of (2.8) is non-zero, is closely related to the "discretely decomposability" of the $\mathfrak{g}$-module ind $\mathfrak{h}_{\mathfrak{h}}^{\mathfrak{g}}\left(V^{\vee}\right)$

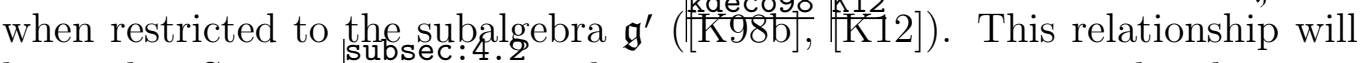
be used in Section in proving that any continuous symmetry breaking operator in a holomorphic setting is given by a differential operator (localness theorem).

(3) Owing to Proposition 2.7 , the left-hand side of $\frac{\text { eqn: }}{2.8)^{\prime}}$ isoo $\mathfrak{h}^{\prime} \subset \mathfrak{h}$. Conversely, if $H^{\prime} \subset H \cap G^{\prime}$, then there is a natural morphism $Y=$

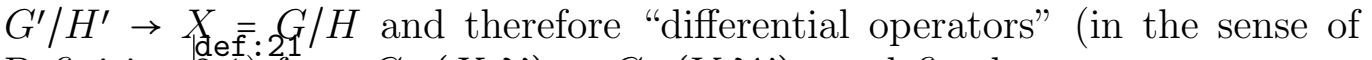
Definition 2.1] from $C^{\infty}(X, \mathcal{V})$ to $C^{\infty}(Y, \mathcal{W})$ are defined.

(4) We shall consider the case where $H^{\prime}=H \cap G^{\prime}$ in later applications, however, Theorem 2.9 also covers the cases where the natural morphism $Y \rightarrow X$ is not injective, i.e. where $H^{\prime} \varsubsetneqq H \cap G^{\prime}$.

An analogous result to Theorem $\frac{t h m: \text { surject }}{2.9}$ holds in the holomorphic setting as well. To be precise, let $G_{\mathbb{C}}$ be a complex Lie group, $G_{\mathbb{C}}^{\prime}, H_{\mathbb{C}}$ and $H_{\mathbb{C}}^{\prime}$ be closed complex subgroups such that $H_{\mathbb{C}}^{\prime} \subset H_{\mathbb{C}} \cap G_{\mathbb{C}}^{\prime}$. We write $\mathfrak{g}, \mathfrak{h}, \ldots$ for the Lie algebras of the complex Lie groups $G_{\mathbb{C}}, H_{\mathbb{C}}, \ldots$, respectively. Given finite-dimensional holomorphic representations $V$ of $H_{\mathbb{C}}$ and $W$ of $H_{\mathbb{C}}^{\prime}$, we form holomorphic vector bundles $\mathcal{V}:=$ $G_{\mathbb{C}} \times_{H_{\mathbb{C}}} V$ over $X_{\mathbb{C}}=G_{\mathbb{C}} / H_{\mathbb{C}}$ and $\mathcal{W}:=G_{\mathbb{C}}^{\prime} \times_{H_{\mathbb{C}}^{\prime}} W$ over $Y_{\mathbb{C}}=G_{\mathbb{C}}^{\prime} / H_{\mathbb{C}}^{\prime}$. 
For simplicity, we assume that $H_{\mathbb{C}}^{\prime}$ is connected. (This is always the case if $G_{\mathbb{C}}^{\prime}$ is a connected complex reductive Lie group and $H_{\mathbb{C}}^{\prime}$ is a parabolic subgroup of $G_{\mathbb{C}}^{\prime}$.) Then we have:

prop:2.10 Theorem 2.12 (Duality theorem in the holomorphic setting). There is a canonical linear isomorphism:

$$
D_{X \rightarrow Y}: \operatorname{Hom}_{\mathfrak{g}^{\prime}}\left(\operatorname{ind}_{\mathfrak{h}^{\prime}}^{\mathfrak{g}^{\prime}}\left(W^{\vee}\right), \operatorname{ind}_{\mathfrak{h}}^{\mathfrak{g}}\left(V^{\vee}\right)\right) \stackrel{\sim}{\longrightarrow} \operatorname{Difff}_{G_{\mathbb{C}}^{\prime}}^{\text {hol }}\left(\mathcal{V}_{X_{\mathbb{C}}}, \mathcal{W}_{Y_{\mathbb{C}}}\right) .
$$

Suppose furthermore that $G, G^{\prime}, H$ and $H^{\prime}$ are real forms of the complex Lie groups $G_{\mathbb{C}}, G_{\mathbb{C}}^{\prime}, H_{\mathbb{C}}$ and $H_{\mathbb{C}}^{\prime}$, respectively. We regard $V$ and $W$ as $H$ - and $H^{\prime}$-modules by the restriction, and form vector bundles $\mathcal{V}=G \times_{H} V$ over $X=G / H$ and $\mathcal{W}=G^{\prime} \times_{H^{\prime}} W$ over $Y=G^{\prime} / H^{\prime}$.

We ask whether or not all symmetry breaking operators have holomorphic extensions. Here is a simple sufficient condition:

cor:holoreal Corollary 2.13. If $H^{\prime}$ is contained in the connected complexification $H_{\mathbb{C}}^{\prime}$, then we have a natural bijection:

$$
\operatorname{Diff}_{G_{\mathbb{C}}^{\prime}}^{\text {hol }}\left(\mathcal{V}_{X_{\mathbb{C}}}, \mathcal{W}_{Y_{\mathbb{C}}}\right) \stackrel{\sim}{\rightarrow} \operatorname{Diff}_{G^{\prime}}\left(\mathcal{V}_{X}, \mathcal{W}_{Y}\right)
$$

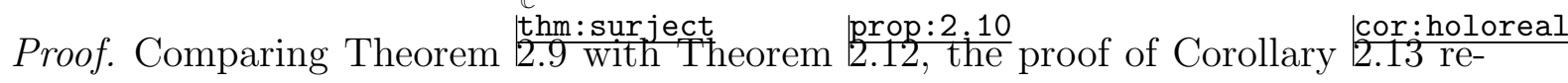
duces to the surjectivity of the inclusion

eqn: gHhom

$$
\operatorname{Hom}_{\left(\mathfrak{g}^{\prime}, H^{\prime}\right)}\left(\operatorname{ind}_{\mathfrak{h}^{\prime}}^{\mathfrak{g}^{\prime}}\left(W^{\vee}\right), \operatorname{ind}_{\mathfrak{h}}^{\mathfrak{g}}\left(V^{\vee}\right)\right) \hookrightarrow \operatorname{Hom}_{\mathfrak{g}^{\prime}}\left(\operatorname{ind}_{\mathfrak{h}^{\prime}}^{\mathfrak{g}^{\prime}}\left(W^{\vee}\right), \operatorname{ind}_{\mathfrak{h}}^{\mathfrak{g}}\left(V^{\vee}\right)\right)
$$

We note that $\operatorname{Hom}_{\left(\mathfrak{g}^{\prime}, H_{\mathbb{C}}^{\prime}\right)}\left(\operatorname{ind}_{\mathfrak{h}^{\prime}}^{\mathfrak{g}^{\prime}}\left(W^{\vee}\right), \operatorname{ind}_{\mathfrak{h}}^{\mathfrak{g}}\left(V^{\vee}\right)\right)$ is a subspace of the left-hand side of

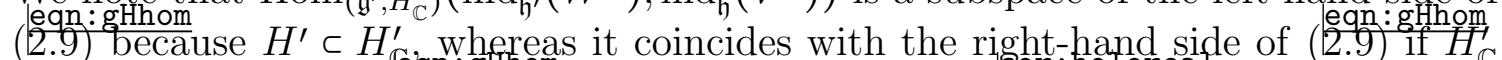
is connected. Hence 2.9] is surjective. Thus Corollary 2.13 is proved.

The rest of this section is devoted to the proof of Theorem 2.9 : surject since the argument is parallel to that of Theorem 2.9 , we omit the proof.

subsec:DXY

2.4. Construction of $D_{t} D_{x \rightarrow} Y_{c t}$ This subsection gives the definition of the linear map $D_{X \rightarrow Y}$ in Theorem 2.9 .

Consider two actions $d R$ and $d L$ of the universal enveloping algebra $U(\mathfrak{g})$ on the space $C^{\infty}(G)$ of smooth complex-valued functions on $G$ induced by the regular representation $L \times R$ of $G \times G$ on $C^{\infty}(G)$ :

eqn : $d R$

$$
(d R(Z) f)(x):=\left.\frac{d}{d t}\right|_{t=0} f\left(x e^{t Z}\right) \text { and }(d L(Z) f)(x):=\left.\frac{d}{d t}\right|_{t=0} f\left(e^{-t Z} x\right),
$$

for $Z \in \mathfrak{g}(\mathbb{R})$.

The right differentiation $\frac{\text { agn: } \mathrm{dR}}{2.10)}$ defines a bilinear map

$$
\Phi: C^{\infty}(G) \times U(\mathfrak{g}) \rightarrow C^{\infty}(G), \quad(F, u) \mapsto d R(u) F,
$$


with the following properties

\begin{tabular}{l|}
\hline eqn:Phi1 \\
\hline eqn:Phi2
\end{tabular}

$$
\begin{aligned}
\Phi(L(g) F, u) & =L(g) \Phi(F, u), \\
\Phi\left(F, u^{\prime} u\right) & =d R\left(u^{\prime}\right) \Phi(F, u),
\end{aligned}
$$

for any $g \in G$ and $u, u^{\prime} \in U(\mathfrak{g})$.

Combining $\Phi$ with the canonical pairing $V \times V^{\vee} \rightarrow \mathbb{C}$, we obtain a bilinear map

$$
\Phi_{V}: C^{\infty}(G) \otimes V \times U(\mathfrak{g}) \otimes_{\mathbb{C}} V^{\vee} \rightarrow C^{\infty}(G) .
$$

Then we have the following:

Lemma 2.14. The map $\Phi_{V}$ induces a well-defined diagram of maps:

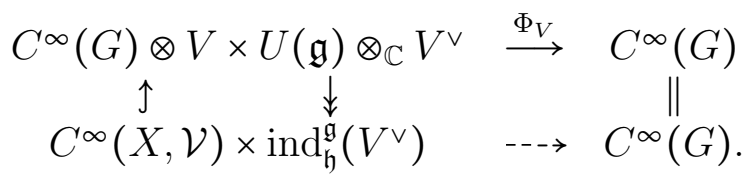

Proof. Denote by $\lambda^{\vee}$ the contragredient representation of the representation $(\lambda, V)$ of $H$, and by $d \lambda^{\vee}$ the infinitesimal representation of $\mathfrak{h}$. The kernel of the natural quotient map $U(\mathfrak{g}) \otimes_{\mathbb{C}} V^{\vee} \rightarrow \operatorname{ind}_{\mathfrak{h}}^{\mathfrak{g}}\left(V^{\vee}\right)$ is generated by

$$
-u Y \otimes v^{\vee}+u \otimes d \lambda^{\vee}(Y) v^{\vee}
$$

with $u \in U(\mathfrak{g}), Y \in \mathfrak{h}$ and $v^{\vee} \in V^{\vee}$. Hence it suffices to show

$$
\Phi_{V}\left(f,-u Y \otimes v^{\vee}+u \otimes d \lambda^{\vee}(Y) v^{\vee}\right)=0
$$

for any $f \in C^{\infty}(X, \mathcal{V}) \simeq C^{\infty}(G, V)^{H}$.

Since $f \in C^{\infty}(G, V)^{H}$ satisfies $d R(Y) f=-d \lambda(Y) f$ for $Y \in \mathfrak{h}$, we have

$$
\begin{aligned}
\Phi_{V}\left(f, u Y \otimes v^{\vee}\right) & =\left\langle d R(u) d R(Y) f, v^{\vee}\right\rangle \\
& =\left\langle d R(u) f, d \lambda^{\vee}(Y) v^{\vee}\right\rangle \\
& =\Phi_{V}\left(f, u \otimes d \lambda^{\vee}(Y) v^{\vee}\right\rangle .
\end{aligned}
$$

Thus the lemma is proved.

lem: indPhi

eqn : CVind
Lemma 2.15. 1) The bilinear map

$$
C^{\infty}(X, \mathcal{V}) \times \operatorname{ind}_{\mathfrak{h}}^{\mathfrak{g}}\left(V^{\vee}\right) \rightarrow \mathbb{C},(f, m) \mapsto \Phi_{V}(f, m)(e)
$$

is $(\mathfrak{g}, H)$-invariant.

2) If $m \in \operatorname{ind}_{\mathfrak{h}}^{\mathfrak{g}}\left(V^{\vee}\right)$ satisfies $\Phi_{V}(f, m)(e)=0$ for all $f \in C^{\infty}(X, \mathcal{V})$ then $m=0$.

Proof. 1) Let $f \in C^{\infty}(X, \mathcal{V})$ and $m \in \operatorname{ind}_{\mathfrak{h}}^{\mathfrak{g}}\left(V^{\vee}\right)$. It follows from $\frac{\text { eqn:Phi1 }}{2.11) \text { and }(2.12) \text { that }}$

$$
\begin{aligned}
\Phi_{V}(d L(Z) f, m) & =d L(Z) \Phi_{V}(f, m) \\
\Phi_{V}(f, Z m) & =d R(Z) \Phi_{V}(f, m)
\end{aligned}
$$


for any $Z \in \mathfrak{g}$. Since

$$
(d L(Z)+d R(Z)) F(e)=0
$$

for any $F \in C^{\infty}(G)$, we have shown the $\mathfrak{g}$-invariance of the bilinear map $\frac{\text { ann } \cdot \mathrm{CV} \text { ind }}{2.13):}$

$$
\Phi_{V}(d L(Z) f, m)(e)+\Phi_{V}(f, Z m)(e)=0 .
$$

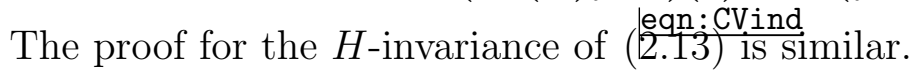

2) Take a basis $\left\{v_{1}, \cdots, v_{k}\right\}$ of $V$, and let $\left\{v_{1}^{\vee}, \cdots, v_{k}^{\vee}\right\}$ be the dual basis in $V^{\vee}$. Choose a complementary subspace $\mathfrak{q}$ of $\mathfrak{h}$ in $\mathfrak{g}$, and fix a basis $\left\{X_{1}, \cdots, X_{n}\right\}$ of $\mathfrak{q}$. Then by the Poincaré-Birkhoff-Witt theorem, we can write $m \in \operatorname{ind}_{\mathfrak{h}}^{\mathfrak{g}}\left(V^{\vee}\right)$ as a finite sum:

$$
m=\sum_{j=1}^{k} \sum_{\alpha=\left(\alpha_{1}, \cdots, \alpha_{n}\right)} a_{\alpha, j} X_{1}^{\alpha_{1}} \cdots X_{n}^{\alpha_{n}} v_{j}^{\vee} .
$$

If $m$ is non-zero, we can find a multi-index $\beta$ and $j_{o}\left(1 \leq j_{o} \leq k\right)$ such that $a_{\beta, j_{o}} \neq 0$ and that $a_{\alpha, j_{0}}=0$ for any multi-index $\alpha$ satisfying $|\alpha|>|\beta|$ and for any $j$. Here $|\alpha|=\sum_{i=1}^{n} \alpha_{i}$ for $\alpha \in \mathbb{N}^{n}$. We take $f \in C^{\infty}(G, V)^{H} \simeq C^{\infty}(X, \mathcal{V})$ such that $f$ is given in a right $H$-invariant neighborhood of $H$ in $G$ by

$$
f\left(\exp \left(\sum_{i=1}^{n} x_{i} X_{i}\right) h\right)=x^{\beta} \lambda(h)^{-1} v_{j_{o}} \quad \text { for } x=\left(x_{1}, \cdots, x_{n}\right) \in \mathbb{R}^{n} \text { and } h \in H .
$$

Then $\Phi_{V}(f, m)(e)=a_{\beta, j_{o}} \beta_{1} ! \cdots \beta_{k} ! \neq 0$. The contraposition completes the proof.

We regard $C^{\infty}(G)$ as a $G \times \mathfrak{g}$-module via the $(L \times d R)$-action. Then the space

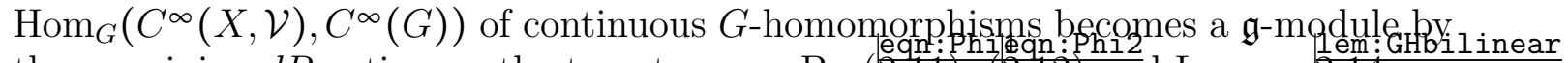
the remaining $d R$-action on the target space. By $(2.11), 2.12)$ and Lemma $\frac{2.14 \text { we }}{2.14}$ get the following $\mathfrak{g}$-homomorphism:

$$
\operatorname{ind}_{\mathfrak{h}}^{\mathfrak{g}}\left(V^{\vee}\right) \longrightarrow \operatorname{Hom}_{G}\left(C^{\infty}(X, \mathcal{V}), C^{\infty}(G)\right), \quad u \otimes v^{\vee} \mapsto\left(f \mapsto\left\langle d R(u) f, v^{\vee}\right\rangle\right) .
$$

Furthermore, it is actually a $(\mathfrak{g}, H)$-homomorphism, where the group $H$ acts on $\operatorname{ind}_{\mathfrak{h}}^{\mathfrak{g}}\left(V^{\vee}\right)=U(\mathfrak{g}) \otimes_{U(\mathfrak{h})} V^{\vee}$ diagonally and acts on $\operatorname{Hom}_{G}\left(C^{\infty}(X, \mathcal{V}), C^{\infty}(G)\right)$ via the $R$-action on $C^{\infty}(G)$.

Let $H^{\prime}$ be a connected closed Lie subgroup of $G$. Given a finite-dimensional representation $W$ of $H^{\prime}$, we form a homogeneous vector bundle $\mathcal{W}_{Z} \equiv \mathcal{W}:=G \times_{H^{\prime}} W$ over $Z:=G / H^{\prime}$.

Taking the tensor product of the $(\mathfrak{g}, H)$-modules in $\left(\frac{29 n: 1234}{2.14}\right.$ with the $H^{\prime}$-module $W$, we get an $\left(H^{\prime} \times(\mathfrak{g}, H)\right)$-homomorphism:

$$
\operatorname{Hom}_{\mathbb{C}}\left(W^{\vee}, \operatorname{ind}_{\mathfrak{h}}^{\mathfrak{g}}\left(V^{\vee}\right)\right) \longrightarrow \operatorname{Hom}_{G}\left(C^{\infty}(X, \mathcal{V}), C^{\infty}(G, W)\right) .
$$

Let $\Delta\left(H^{\prime}\right)$ be a subgroup of $H^{\prime} \times H$ defined by $\left\{(h, h): h \in H^{\prime}\right\}$. Taking $\Delta\left(H^{\prime}\right)$ invariants, we obtain the following $\mathbb{C}$-linear map:

$$
\operatorname{Hom}_{H^{\prime}}\left(W^{\vee}, \operatorname{ind}_{\mathfrak{h}}^{\mathfrak{g}}\left(V^{\vee}\right)\right) \longrightarrow \operatorname{Hom}_{G}\left(C^{\infty}(X, \mathcal{V}), C^{\infty}(Z, \mathcal{W})\right), \quad \varphi \mapsto D_{\varphi}
$$


where $D_{\varphi}$ satisfies

eqn:Dfw (2.16)

$$
\left\langle D_{\varphi} f, w^{\vee}\right\rangle=\Phi_{V}\left(f, \varphi\left(w^{\vee}\right)\right)
$$

for any $f \in C^{\infty}(X, \mathcal{V})$ and any $w^{\vee} \in W^{\vee}$.

Remark 2.16. If $H^{\prime}$ is connected, then we can replace $H_{H^{\prime}}$ by $H_{o m}$ in $\frac{\text { egn }}{2.150 r f}$

lem:Ding Lemma 2.17. The map (2.15) is injective.

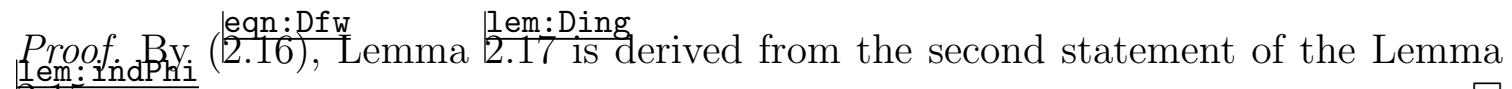
2.15 .

Take any subgroup $G^{\prime}$ of $G$ containing $H^{\prime}$ and form a homogeneous vector bundle $\mathcal{W}_{Y}:=G^{\prime} \times_{H^{\prime}} W$ over $Y=G^{\prime} / H^{\prime}$. Then, the vector bundle $\mathcal{W}_{Y}$ is isomorphic to the restriction $\left.\mathcal{W}_{Z}\right|_{Y}$ of the vector bundle $\mathcal{W}_{Z}$ to the submanifold $Y$ of the base space $Z$. Let

$$
R_{Z \rightarrow Y}: C^{\infty}\left(Z, \mathcal{W}_{Z}\right) \rightarrow C^{\infty}\left(Y, \mathcal{W}_{Y}\right)
$$

be the restriction map of sections. For $\varphi \in \operatorname{Hom}_{\mathfrak{h}^{\prime}}\left(W^{\vee}, \operatorname{ind}_{\mathfrak{h}}^{\mathfrak{g}}\left(V^{\vee}\right)\right)$ we set

$$
D_{X \rightarrow Y}(\varphi):=R_{Z \rightarrow Y} \circ D_{\varphi}
$$

Then $D_{X \rightarrow Y}(\varphi): C^{\infty}(X, \mathcal{V}) \rightarrow C^{\infty}(Y, \mathcal{W})$ is a $G^{\prime}$-equivariant differential operator, ie $e_{\text {: }}$ surject $D_{X \rightarrow Y}$ defines a linear map $\operatorname{Hom}_{\mathfrak{h}^{\prime}}\left(W^{\vee}, \operatorname{ind}_{\mathfrak{h}}^{\mathfrak{g}}\left(V^{\vee}\right)\right) \rightarrow \operatorname{Diff}_{G^{\prime}}\left(\mathcal{V}_{X}, \mathcal{W}_{Y}\right)$. Theorem 2.9 describes explicitly the image $D_{X \rightarrow Y}$ when $H^{\prime} \subset H \cap G^{\prime}$, namely, when the following diagram exists:

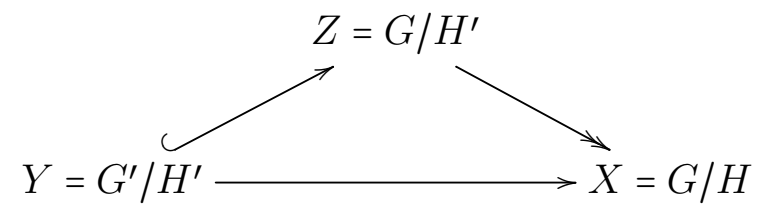

Remark 2.18. The left-hand side of (2.8) isoo does not depend on the choice of $G^{\prime}$. This fact is reflected by the commutativity of the following diagram.

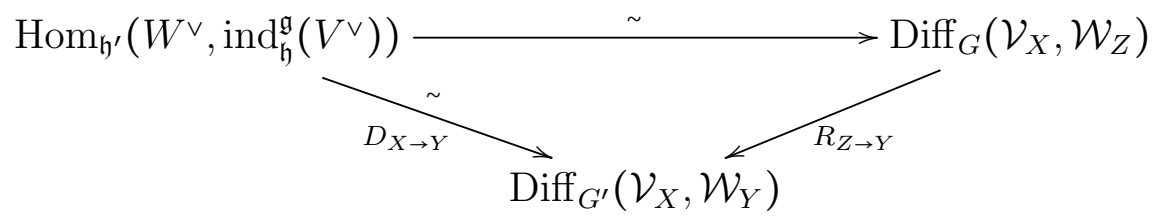

2.5. Proof of Theorem 2.9. 2 . We have already seen in Lemma 2.17 that $D_{X \rightarrow Y}$ is injective. In order to prove the surjectivity of the linear map $D_{X \rightarrow Y}$, we realize the induced $U(\mathfrak{g})$-module $\operatorname{ind}_{\mathfrak{h}}^{\mathfrak{g}}\left(V^{\vee}\right)$ in the space of distributions.

We recall that $\mathcal{V}^{*}=\mathcal{V}^{\vee} \otimes \Omega_{X}$ is the dualizing bundle of a vector bundle $\mathcal{V}$ over $X$. For a closed subset $S$ and an open subset $U$ in $X$ containing $S$, we write $\mathcal{D}_{S}^{\prime}\left(U, \mathcal{V}^{*}\right)$ for 
the space of $\mathcal{V}^{*}$-valued distributions on $U$ with support in $S$. Obviously, $\mathcal{D}_{S}^{\prime}\left(U, \mathcal{V}^{*}\right)=$ $\mathcal{D}_{S}^{\prime}\left(X, \mathcal{V}^{*}\right)$. If $S$ is compact, then $\mathcal{D}_{S}^{\prime}\left(U, \mathcal{V}^{*}\right)$ is contained in the space $\mathcal{E}^{\prime}\left(U, \mathcal{V}^{*}\right)$ of distributions on $U$ with compact support, and thus coincides with $\mathcal{E}_{S}^{\prime}\left(U, \mathcal{V}^{*}\right):=$ $\mathcal{D}_{S}^{\prime}\left(U, \mathcal{V}^{*}\right) \cap \mathcal{E}^{\prime}\left(U, \mathcal{V}^{*}\right)$

We return to the setting of Theorem 2.9 , where $\mathcal{V}$ is a $G$-equivariant vector bundle over $X=G / H$. Then the Lie group $G$ acts on $C^{\infty}(X, \mathcal{V})$ and $\mathcal{E}^{\prime}\left(X, \mathcal{V}^{*}\right)$ by the pull-back of smooth sections and distributions, respectively. The infinitesimal action defines representations of the Lie algebra $\mathfrak{g}$ on $C^{\infty}(U, \mathcal{V})$ and $\mathcal{E}_{S}^{\prime}\left(U, \mathcal{V}^{*}\right)$.

The "integration map" (2.2)

eqn : intE13

$$
\mathcal{E}^{\prime}\left(X, \Omega_{X}\right) \rightarrow \mathbb{C}, \quad \omega \mapsto \int_{X} \omega
$$

is $G$-invariant. Composing this with the $G$-invariant bilinear map (contraction):

$$
C^{\infty}(X, \mathcal{V}) \times \mathcal{E}^{\prime}\left(X, \mathcal{V}^{*}\right) \longrightarrow \mathcal{E}^{\prime}\left(X, \Omega_{X}\right), \quad(f, h) \mapsto\langle f, h\rangle,
$$

we obtain the following $G$-invariant bilinear form

$$
C^{\infty}(X, \mathcal{V}) \times \mathcal{E}^{\prime}\left(X, \mathcal{V}^{*}\right) \longrightarrow \mathbb{C}, \quad(f, h) \mapsto \int_{X}\langle f, h\rangle
$$

Similarly, we obtain the following local version:

lem:EHom Lemma 2.19. Let $S$ be a closed subset of $X$ and $U$ an open neighborhood of $S$ in $X$. Then, we have the natural $\mathfrak{g}$-invariant bilinear form:

$$
C^{\infty}(U, \mathcal{V}) \times \mathcal{E}_{S}^{\prime}\left(U, \mathcal{V}^{*}\right) \longrightarrow \mathbb{C}, \quad(f, h) \mapsto \int_{U}\langle f, h\rangle
$$

Moreover, if $S \subset U$ are both $H$-invariant subsets in $X$, then the bilinear form is also $H$-invariant.

We write $o=e H \in X$ for the origin. By Lemmas 2.15 and 2.19 , we have obtained two $(\mathfrak{g}, H)$-invariant pairings:

$$
\begin{aligned}
C^{\infty}(X, \mathcal{V}) \times \operatorname{ind}_{\mathfrak{h}}^{\mathfrak{g}}\left(V^{\vee}\right) & \longrightarrow \mathbb{C}, \quad(f, m) \mapsto \Phi_{V}(f, m)(e), \\
C^{\infty}(X, \mathcal{V}) \times \mathcal{E}_{\{o\}}^{\prime}\left(X, \mathcal{V}^{*}\right) & \longrightarrow \mathbb{C} \quad(f, h) \mapsto \int_{X}\langle f, h\rangle .
\end{aligned}
$$

Let us show that there is a natural $(\mathfrak{g}, H)$-isomorphism between $\operatorname{ind}_{\mathfrak{h}}^{\mathfrak{g}}\left(V^{\vee}\right)$ and $\mathcal{E}_{\{o\}}^{\prime}\left(X, \mathcal{V}^{*}\right)$. In fact, it follows from Lemma 2.15 that there exists an injective $(\mathfrak{g}, H)$ homomorphism

$$
A: \operatorname{ind}_{\mathfrak{h}}^{\mathfrak{g}}\left(V^{\vee}\right) \rightarrow \mathcal{E}_{\{o\}}\left(X, \mathcal{V}^{*}\right)
$$

such that

$$
\Phi_{V}(f, m)(e)=\int_{X}\langle f, A(m)\rangle \text { for all } m \in \operatorname{ind}_{\mathfrak{h}}^{\mathfrak{g}}\left(V^{\vee}\right) \text { and } f \in C^{\infty}(X, \mathcal{V})
$$


For a homogeneous vector bundle $\mathcal{V}=G \times_{H} V$ we define a vector-valued Dirac $\delta$-function $\delta \otimes v^{\vee} \in \mathcal{E}_{\{o\}}^{\prime}\left(X, \mathcal{V}^{*}\right)$, for $v \in V^{\vee}$ by

eqn: deltaV

$$
\left\langle f, \delta \otimes v^{\vee}\right\rangle:=\left\langle f(e), v^{\vee}\right\rangle \text { for } f \in C^{\infty}(X, \mathcal{V}) \simeq C^{\infty}(G, V)^{H} .
$$

By the definition of $\Phi_{V}$, we have

$$
\Phi_{V}\left(f, 1 \otimes v^{\vee}\right)(e)=\left\langle f(e), v^{\vee}\right\rangle .
$$

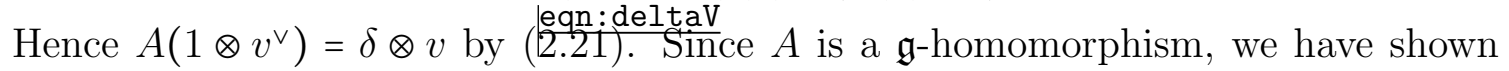
that

$$
A\left(u \otimes v^{\vee}\right)=d L(u)\left(\delta \otimes v^{\vee}\right), \quad \text { for } u \in U(\mathfrak{g}), v \in V^{\vee} .
$$

lem:indE

eqn : isoMh

$$
A: \operatorname{ind}_{\mathfrak{h}}^{\mathfrak{g}}\left(V^{\vee}\right) \longrightarrow \mathcal{E}_{\{o\}}^{\prime}\left(X, \mathcal{V}^{*}\right), \quad u \otimes v^{\vee} \mapsto d L(u)\left(\delta \otimes v^{\vee}\right)
$$

is bijective.

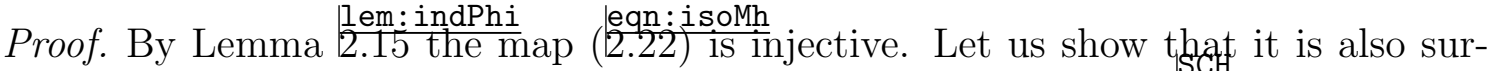
jective. By the structural theorem of (scalar-valued) distributions $\$ \mathbf{S 6 6}$, Chapter III, Théorème XXXVII], distributions supported on the singleton $\{o\}$ are obtained as a finite sum of derivatives of the Dirac's delta function. An analogous statement holds for vector-bundle valued distributions supported on $\{o\}$, as we can see by trivializing the bundle near the origin $o$. Choose a complementary subspace $\mathfrak{q}(\mathbb{R})$ of $\mathfrak{h}(\mathbb{R})=\operatorname{Lie}(H)$ in $\mathfrak{g}(\mathbb{R})=\operatorname{Lie}(G)$. Since $d L(Z)(Z \in \mathfrak{q}(\mathbb{R}))$ spans the tangent space $T_{o}(G / H) \simeq \mathfrak{q}(\mathbb{R})$, any derivative of the vector-valued Dirac's delta function is given as a linear combination of elements of the form $d L(u)\left(\delta \otimes v^{\vee}\right)\left(u \in U(\mathfrak{g}), v^{\vee} \in V^{\vee}\right)$. Thus the map (2.22) is surjective.

Let $\mathbb{C}_{2 \rho}$ denote the one-dimensional representation of $H$ defined by

$$
h \mapsto\left|\operatorname{det}\left(\operatorname{Ad}_{G / H}(h): \mathfrak{g} / \mathfrak{h} \rightarrow \mathfrak{g} / \mathfrak{h}\right)\right|^{-1} .
$$

If $H$ is a parabolic subgroup of $G$ with Langlands decomposition $P=M A N_{+}$then the infinitesimal representation of $\mathbb{C}_{2 \rho}$ is given by the sum of the roots for $\mathfrak{n}_{+}=\operatorname{Lie}\left(N_{+}\right)$. The bundle of densities $\Omega_{G / H}$ is given as a $G$-equivariant line bundle,

$$
\Omega_{G / H} \simeq G \times_{H}\left|\operatorname{det}^{-1} \operatorname{Ad}_{G / H}\right| \simeq G \times_{H} \mathbb{C}_{2 \rho} .
$$

For an $H$-module $(\lambda, V)$, we define a "twist" of the contragredient representation $\lambda_{2 \rho}^{\vee}$ on the dual space $V^{\vee}$ (or simply denoted by $V_{2 \rho}^{\vee}$ ) by the formula

$$
\lambda^{*} \equiv \lambda_{2 \rho}^{\vee}:=\lambda^{\vee} \otimes \mathbb{C}_{2 \rho}=\lambda^{\vee} \otimes\left|\operatorname{det}^{-1} \operatorname{Ad}_{G / H}\right| .
$$

Then the dualizing bundle $\mathcal{V}^{*}=\mathcal{V}^{\vee} \otimes \Omega_{G / H}$ of the vector bundle $\mathcal{V}=G \times_{H} V$ is given, as a homogeneous vector bundle, by:

$$
\mathcal{V}^{*} \equiv \mathcal{V}_{2 \rho}^{\vee} \simeq G \times_{H} V_{2 \rho}^{\vee}
$$


Then $\mathcal{D}^{\prime}\left(X, \mathcal{V}^{*}\right)$ is identified with

$$
\left(\mathcal{D}^{\prime}(G) \otimes V_{2 \rho}^{\vee}\right)^{\Delta(H)}=\left\{F \in \mathcal{D}^{\prime}(G) \otimes V^{\vee}: \lambda_{2 \rho}^{\vee}(h) F(\cdot h)=F(\cdot) \quad \text { for any } h \in H\right\} .
$$

Now let us consider the setting of Theorem $\frac{\Delta \text { thm }}{2.9}$ : surject where we have a $G^{\prime}$-equivariant (but not necessarily injective) morphism from $Y=G^{\prime} / H^{\prime}$ to $X=G / H$.

lem:A Lemma 2.21. Suppose that $G^{\prime}$ is a subgroup of $G$. Then the multiplication map

$$
m: G \times G^{\prime} \rightarrow G, \quad\left(g, g^{\prime}\right) \mapsto\left(g^{\prime}\right)^{-1} g,
$$

induces the isomorphism:

$$
m^{*}:\left(\mathcal{D}^{\prime}\left(X, \mathcal{V}^{*}\right) \otimes W\right)^{\Delta\left(H^{\prime}\right)} \stackrel{\sim}{\longrightarrow} \mathcal{D}^{\prime}\left(X \times Y, \mathcal{V}^{*} \otimes \mathcal{W}\right)^{\Delta\left(G^{\prime}\right)}
$$

Proof. The image of the pull-back $m^{*}: \mathcal{D}^{\prime}(G) \rightarrow \mathcal{D}^{\prime}\left(G \times G^{\prime}\right)$ is $\mathcal{D}^{\prime}\left(G \times G^{\prime}\right)^{\Delta\left(G^{\prime}\right)}$, where $G^{\prime}$ acts diagonally from the left. Thus, considering the remaining $G \times G^{\prime}$ action from the right, we take $H \times H^{\prime}$-invariants with respect to the diagonal action in the $\left(G \times G^{\prime} \times H \times H^{\prime}\right)$-isomorphism:

$$
m^{*} \otimes \mathrm{id} \otimes \mathrm{id}: \mathcal{D}^{\prime}(G) \otimes V_{2 \rho}^{\vee} \otimes W \stackrel{\sim}{\longrightarrow} \mathcal{D}^{\prime}\left(G \times G^{\prime}\right)^{\Delta\left(G^{\prime}\right)} \otimes V_{2 \rho}^{\vee} \otimes W,
$$

and therefore we get the lemma.

We recall from Section 2.1 that any continuous linear map $T: C^{\infty}(X, \mathcal{V}) \rightarrow$ $C^{\infty}(Y, \mathcal{W})$ is given by a unique distribution kernel $K_{T} \in \mathcal{D}^{\prime}\left(X \times Y, \mathcal{V}^{*} \otimes \mathcal{W}\right)$. The following lemma gives a necessary and sufficient condition on the distribution $K_{T}$ for the linear map $T$ to be a $G^{\prime}$-equivariant differential operator.

lem:312 Lemma 2.22. There is a natural linear isomorphism:

eqn $: \mathrm{KT}$

$$
\operatorname{Diff}_{G^{\prime}}\left(\mathcal{V}_{X}, \mathcal{W}_{Y}\right) \stackrel{\sim}{\longrightarrow}\left(\mathcal{D}_{\{o\}}^{\prime}\left(X, \mathcal{V}^{*}\right) \otimes W\right)^{\Delta\left(H^{\prime}\right)}, \quad T \mapsto\left(m^{*}\right)^{-1}\left(K_{T}\right) .
$$

Proof. First, we show that the map $(2.24)$ is well-defined. Suppose $T \in \operatorname{Diff}_{G^{\prime}}\left(\mathcal{V}_{X}, \mathcal{W}_{Y}\right)$. Since $K_{T}$ is uniquely determined by $T$, the operator $T$ is $G^{\prime}$-equivariant, i.e. $L(g) \circ$ To $L_{L}\left(g^{-1}\right)=T$ for all $g \in G^{\prime}$ if and only if $K_{T} \in \mathcal{D}^{\prime}\left(X \times Y, \mathcal{V}^{*} \otimes \mathcal{W}\right)^{\Delta\left(G^{\prime}\right)}$. By Lemma 2.3 the distribution kernel $K_{T}$ is supported on the diagonal set $\Delta(Y)=\{(p(y), y)$ : $y \in Y\} \subset X \times Y$. Via the bijection $m^{*}$ given in Lemma 2.21 we thus have

$$
\operatorname{Supp}\left(\left(m^{*}\right)^{-1} K_{T}\right) \subset\{o\} .
$$

Hence the map $\frac{(2.24) \cdot K T}{2.24}$ is well-defined. The injectivity of $\left(\frac{\operatorname{ean} \cdot \mathrm{KT}}{2.24}\right)$ is clear.

Conversely, take any element $k \in\left(\mathcal{D}_{\{o\}}^{\prime}\left(X, \mathcal{V}^{*}\right) \otimes W\right)^{\Delta\left(H^{\prime}\right)}$. We set $K:=m^{*}(k) \epsilon$ $\mathcal{D}^{\prime}\left(X \times Y, \mathcal{V}^{*} \otimes \mathcal{W}\right)^{\Delta\left(G^{\prime}\right)}$, and define a linear map

$$
T: C^{\infty}(X, \mathcal{V}) \longrightarrow \mathcal{D}^{\prime}(Y, \mathcal{W}), \quad f \mapsto \int_{X} f(x) K(x, \cdot) .
$$

Then $T$ is $G^{\prime}$-equivariant because $K$ is $\Delta\left(G^{\prime}\right)$-invariant. 
Let us show that $T f \in C^{\infty}(Y, \mathcal{W})$ for any $f \in C^{\infty}(X, \mathcal{V})$. To see this, we take neighborhoods $U, U^{\prime}$ and $U^{\prime \prime}$ of $x_{o}=p\left(y_{o}\right)$ in $X, y_{o}$ in $Y$, and $e$ in $G^{\prime}$, respectively, such that $g U^{\prime} \subset p^{-1}(U)$ for any $g \in U^{\prime \prime}$. Since the kernel $K$ is supported on the diagonal set $\Delta(Y),\left.T F\right|_{U^{\prime}}$ is locally of the form $\frac{2.40}{2.4}$ as in the proof of Lemma 2.3 .1

Since $T$ is $G^{\prime}$-equivariant, we have

$$
\sum_{\alpha} h_{\alpha}(y) \frac{\partial^{|\alpha|} f}{\partial x^{\alpha}}(g p(y))=\sum_{\alpha} h_{\alpha}(g y) \frac{\partial^{|\alpha|} f}{\partial x^{\alpha}}(p(y)),
$$

for any $y \in U^{\prime}, g \in U^{\prime \prime}$, and $f \in C^{\infty}(U) \otimes V$. By taking $f(x)=x^{\alpha} \otimes v\left(\alpha \in \mathbb{N}^{n}\right.$ and $v \in V)$ as test functions, there are some $\varphi_{\alpha \beta} \in C^{\infty}\left(U^{\prime \prime} \times U^{\prime}\right)$ for $|\beta|<|\alpha|$ such that

$$
h_{\alpha}(g y)=h_{\alpha}(y)+\sum_{|\beta|<|\alpha|} \varphi_{\alpha \beta}(g, y) h_{\beta}(y) .
$$

Therefore we see inductively on $|\alpha|$ that $h_{\alpha}(y) \in C^{\infty}\left(U^{\prime}\right) \otimes \operatorname{Hom}(V, W)$ for all $\alpha$ because $G^{\prime}$ acts transitively on $Y$. Hence $\left.T f\right|_{U^{\prime}} \in C^{\infty}\left(U^{\prime}\right) \otimes W$. Thus we have shown that $T$ maps $C^{\infty}(X, \mathcal{V})$ into $C^{\infty}(Y, \mathcal{Y})$.

Finally, it follows from Lemma 2.3 that $T$ is a differential operator because $\operatorname{Supp} K \subset$ $\Delta(Y)$. Now we have proved the lemma.

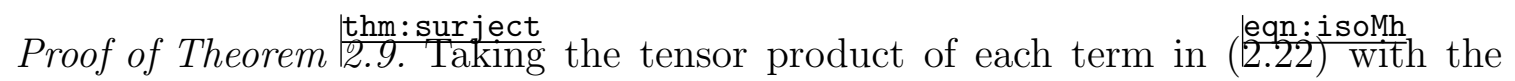
finite-dimensional representation $W$ of $H^{\prime}$, we get a bijection between the subspaces of $\mathfrak{h}$ '-invariants:

$$
\operatorname{Hom}_{\mathfrak{h}^{\prime}}\left(W^{\vee}, \operatorname{ind}_{\mathfrak{h}}^{\mathfrak{g}}\left(V^{\vee}\right)\right) \stackrel{\sim}{\longrightarrow}\left(\mathcal{D}_{\{o\}}^{\prime}\left(X, \mathcal{V}_{X}^{*}\right) \otimes W\right)^{\Delta\left(H^{\prime}\right)} .
$$

Composing this with the bijection in Lemma 2.22 , we obtain a bijection from

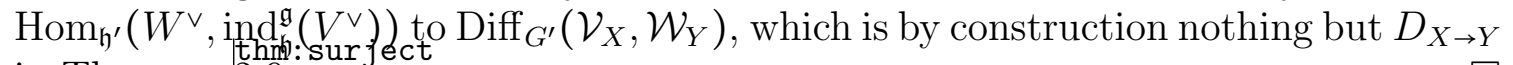
in Theorem 2.9 .

\section{Algebraic Fourier transform for generalized Verma modules}

The duality theorem (Theorem 2.9 ) surject breaking operator $D \in \operatorname{Diff}_{G^{\prime}}\left(\mathcal{V}_{X}, \mathcal{W}_{Y}\right)$, it suffices to find $\varphi \in \operatorname{Hom}_{H^{\prime}}\left(W^{\vee}, \operatorname{ind}_{\mathfrak{h}}^{\mathfrak{g}}\left(V^{\vee}\right)\right)$. In Section 4 , we shall present a new method (F-method) which characterizes the "algebraic Fourier transform" of $\varphi$ as a solution to a certain system of partial differential equations.

In this section we introduce and study. the "algebraic Fourier transform" of generalized Verma modules. Proposition 3.11 is particularly important to the F-method.

3.1. Weyl algebra and algebraic Fourier transform. Let $E$ be a vector space over $\mathbb{C}$. The Weyl algebra $\mathcal{D}(E)$ is the ring of holomorphic differential operators on $E$ with polynomial coefficients. 
def:hat Definition 3.1. We define the algebraic Fourier transform as an isomorphism of two Weyl algebras on $E$ and its dual space $E^{\vee}$ :

$$
\mathcal{D}(E) \rightarrow \mathcal{D}\left(E^{\vee}\right), \quad T \mapsto \widehat{T},
$$

induced by

eqn:FTdiff

$$
\frac{\widehat{\partial}}{\partial z_{j}}:=-\zeta_{j}, \quad \widehat{z}_{j}:=\frac{\partial}{\partial \zeta_{j}}, \quad 1 \leq j \leq n=\operatorname{dim} E .
$$

where $\left(z_{1}, \ldots, z_{n}\right)$ are coordinates on $E$ and $\left(\zeta_{1}, \ldots, \zeta_{n}\right)$ are the dual coordinates on $E^{\vee}$.

Example 3.2. Let $E_{z}:=\sum_{j=1}^{n} z_{j} \frac{\partial}{\partial z_{j}}$ be the Euler operator on E. Then, by the commutation relations

eqn : commrel

$$
\frac{\partial}{\partial \zeta_{i}} \zeta_{j}-\zeta_{j} \frac{\partial}{\partial \zeta_{i}}=\delta_{i j}
$$

in the Weyl algebra $\mathcal{D}\left(E^{\vee}\right)$, where $\delta_{i j}$ is the Kronecker delta. Hence we have $\widehat{E_{z}}=$ $-E_{\zeta}-n$.

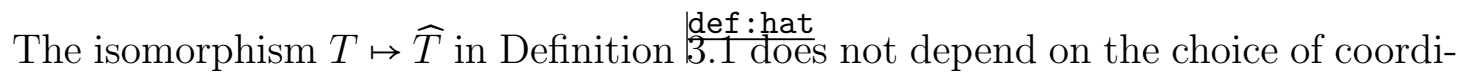
nates. To see this, we consider the natural action of the general linear group $G L(E)$ on $E$, which yields automorphisms of the ring $\operatorname{Pol}(E)$ of polynomials of $E$ and the Weyl algebra $\mathcal{D}(E)$. For $A \in G L(E)$, we set

$$
\begin{aligned}
A_{\#}: & \operatorname{Pol}(E) \longrightarrow \operatorname{Pol}(E), \quad F \mapsto F\left(A^{-1} \cdot\right), \\
A_{*}: & \mathcal{D}(E) \longrightarrow \mathcal{D}(E), \quad T \mapsto A_{\#} \circ T \circ A_{\#}^{-1} .
\end{aligned}
$$

We denote by ${ }^{t} A \in G L\left(E^{\vee}\right)$ the dual map of $A$. Then we have

lem:Faffine Lemma 3.3. For any $A \in G L(E)$ and $T \in \mathcal{D}(E)$,

$$
\widehat{A_{*} T}=\left({ }^{t} A^{-1}\right)_{*} \widehat{T} .
$$

The proof is straightforward from the definition ( $\left.\frac{\text { def }}{3.1}\right)$, hat and we omit it.

Next we consider the group homomorphism $G L(E) \longrightarrow G L(\operatorname{Pol}(E)), A \mapsto A_{\#}$. Taking the differential, we get a Lie algebra homomorphism $\operatorname{End}(E) \rightarrow \mathcal{D}(E)$. In the coordinates, we write $Z={ }^{t}\left(z_{1}, \cdots, z_{n}\right)$ and $\partial_{Z}={ }^{t}\left(\frac{\partial}{\partial z_{1}}, \cdots, \frac{\partial}{\partial z_{n}}\right)$. Then this homomorphism amounts to

eqn : endo1

$$
\Psi_{E}: \operatorname{End}(E) \rightarrow \mathcal{D}(E), \quad A \mapsto-{ }^{t} Z^{t} A \partial_{Z} \equiv-\sum_{i, j} A_{i j} z_{j} \frac{\partial}{\partial z_{i}} .
$$

Let $\sigma: \mathfrak{g} \rightarrow \operatorname{End}(E)$ be a representation of a Lie algebra $\mathfrak{g}$ on $E$, and $\sigma^{\vee}: \mathfrak{g} \rightarrow$ $\operatorname{End}\left(E^{\vee}\right)$ the contragredient representation. Then the algebraic Fourier transform 
$T \mapsto \widehat{T}$ relates the two Lie algebra homomorphisms $\Psi_{E} \circ \sigma: \mathfrak{g} \rightarrow \mathcal{D}(E)$ and $\Psi_{E^{\vee}} \circ \sigma^{\vee}:$ $\mathfrak{g} \rightarrow \mathcal{D}\left(E^{\vee}\right)$ as follows:

\section{rop:Phihat Lemma 3.4.}

$$
\overline{\Psi_{E} \circ \sigma}=\Psi_{E^{\vee}} \circ \sigma^{\vee}+(\text { Trace } \circ \sigma) \cdot \operatorname{id}_{E^{\vee}} .
$$

Proof. In the coordinates, we write $A:=\sigma(Z) \in \operatorname{End}(E) \simeq M(n, \mathbb{C})$ for $Z \in \mathfrak{g}$. Then,

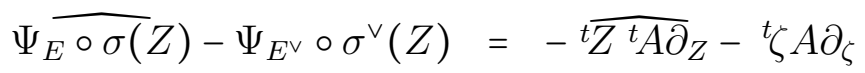

$$
\begin{aligned}
& ={ }^{t} \partial_{\zeta}{ }^{t} A \zeta-{ }^{t} \zeta A \partial_{\zeta} \\
& =(\operatorname{Trace} A) \operatorname{id}_{E^{\vee}} \text {, }
\end{aligned}
$$

where the last equality follows from the commutation relations 3.2 .

For actual computations that will be undertaken in a subsequent paper [PART2 it is convenient to give another interpretation of the algebraic Fourier transform by using real forms of $E$.

Definition 3.5. Fix a real form $E(\mathbb{R})$ of the complex vector space $E$. Let $\mathcal{E}_{\{0\}}^{\prime}(E(\mathbb{R}))$ be the space of distributions on the vector space $E(\mathbb{R})$ supported at the origin 0 . We define a "Fourier transform" $\mathcal{F}_{c}: \mathcal{E}_{\{0\}}^{\prime}(E(\mathbb{R})) \rightarrow \operatorname{Pol}\left(E^{\vee}\right)$ by the following formula:

$$
\mathcal{F}_{c} f(\zeta):=\left\langle f(\cdot), e^{\langle\cdot, \zeta\rangle}\right\rangle=\int_{E(\mathbb{R})} e^{\langle x, \zeta\rangle} f(x) \quad \text { for } \zeta \in E^{\vee} .
$$

We have used the function $e^{\langle x, \zeta\rangle}$ in $\frac{\text { agn }}{3.4}$ algF rather than $e^{-\sqrt{-1}\langle x, \zeta\rangle}$ or $e^{-\langle x, \zeta\rangle}$ which are involved in the usual Fourier transform or the Laplace transform, $\underset{\text { resper }}{\text { respectively. This }}$ convention makes later computations simpler (see Remark 4.2).

Furthermore, with our convention

$$
\mathcal{F}_{c}(f(A \cdot))=\left(\mathcal{F}_{c} f\right)\left({ }^{t} A^{-1} \cdot\right)
$$

for any $A \in G L_{\mathbb{R}}(E(\mathbb{R}))$.

The Fourier transform $\mathcal{F}_{c}$ induces an algebra isomorphism

$$
\mathcal{F}_{c}: \mathcal{E}_{\{0\}}^{\prime}(E(\mathbb{R})) \stackrel{\sim}{\rightarrow} \operatorname{Pol}\left(E^{\vee}\right)
$$

between the polynomial algebra $\operatorname{Pol}\left(E^{\vee}\right)$ with unit $\mathbf{1}$, the constant function on $E^{\vee}$, and the convolution algebra $\mathcal{E}_{\{0\}}^{\prime}(E(\mathbb{R}))$ with unit $\delta$, the Dirac delta function. We write $\mathcal{F}_{c}^{-1}: \operatorname{Pol}\left(E^{\vee}\right) \stackrel{\sim}{\rightarrow} \mathcal{E}_{\{0\}}^{\prime}(E(\mathbb{R}))$ for the inverse "Fourier transform":

$$
\mathcal{F}_{c}^{-1}(\mathbf{1})=\delta
$$


rem:45 Remark 3.6. The Weyl algebra $\mathcal{D}(E)$ acts naturally on the space of distributions on $E(\mathbb{R})$, and in particular, on $\mathcal{E}_{\{0\}}^{\prime}(E(\mathbb{R}))$. The algebraic Fourier transform defined in Definition 3.1 satisfies

eqn :F-trans

$$
\widehat{T}=\mathcal{F}_{c} \circ T \circ \mathcal{F}_{c}^{-1} \quad \text { for } T \in \mathcal{D}(E),
$$

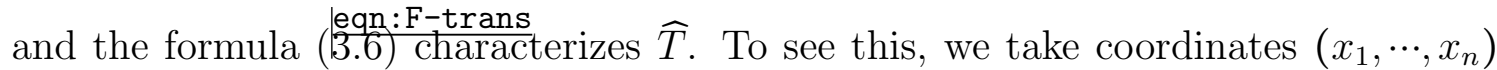
on $E(\mathbb{R})$, and extend them to the complex coordinates $\left(z_{1}, \cdots, z_{n}\right)$ on $E$ and the dual ones $\left(\zeta_{1}, \cdots, \zeta_{n}\right)$ on $E^{\vee}$. Let $P(\zeta)=\zeta^{\alpha} \in \operatorname{Pol}\left(E^{\vee}\right)$ and $T=\sum_{\beta, \gamma} a_{\beta, \gamma} z^{\beta} \frac{\partial^{|\gamma|}}{\partial z^{\gamma}} \in \mathcal{D}(E)$. Then we have

$$
\widehat{T} P=\sum_{\beta, \gamma}(-1)^{|\gamma|} a_{\beta, \gamma} \frac{\partial^{|\beta|}}{\partial z^{\beta}} \zeta^{\alpha+\gamma},
$$

and on the other hand,

$$
\begin{aligned}
\mathcal{F}_{c} \circ T \circ \mathcal{F}_{c}^{-1} P & =(-1)^{|\alpha|} \mathcal{F}_{c} \circ T\left(\delta^{\alpha}(x)\right)=(-1)^{|\alpha|} \mathcal{F}_{c}\left(\sum_{\beta, \gamma} a_{\beta, \gamma} x^{\beta} \delta^{\alpha+\gamma}(x)\right) \\
& =(-1)^{|\alpha|} \sum_{\beta, \gamma}(-1)^{|\alpha|+|\gamma|} a_{\beta, \gamma} \frac{\partial^{|\beta|}}{\partial z^{\beta}} \zeta^{\alpha+\gamma} .
\end{aligned}
$$

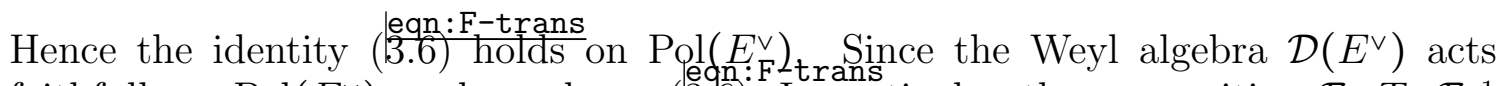
faithfully on $\operatorname{Pol}\left(E^{\vee}\right)$, we have shown $(3.6)$. In particular, the composition $\mathcal{F}_{c} \circ T \circ \mathcal{F}_{c}^{-1}$ does not depend on the choice of a real form $E(\mathbb{R})$.

\subsection{Holomorphic vector fields associated to the Gelfand-Naimark decom-}

position. It is convenient to prepare some notation in the complex reductive Lie algebras for later purpose.

Let $\mathfrak{g}$ be a complex reductive Lie algebra, and $\mathfrak{p}=\mathfrak{l}+\mathfrak{n}_{+}$a Levi decomposition of a parabolic subalgebra. Let $G_{\mathbb{C}}$ be a connected complex Lie group with Lie algebra $\mathfrak{g}$, and $P_{\mathbb{C}}=L_{\mathbb{C}} \exp \mathfrak{n}_{+}$the parabolic subgroup with Lie algebra $\mathfrak{p}=\mathfrak{l}+\mathfrak{n}_{+}$. According to the Gelfand-Naimark decomposition $\mathfrak{g}=\mathfrak{n}_{-}+\mathfrak{l}+\mathfrak{n}_{+}$of the Lie algebra $\mathfrak{g}$, we have a diffeomorphism

$$
\mathfrak{n}_{-} \times L_{\mathbb{C}} \times \mathfrak{n}_{+} \rightarrow G_{\mathbb{C}}, \quad(Z, \ell, Y) \mapsto(\exp Z) \ell(\exp Y),
$$

into an open dense subset $G_{\mathbb{C}}^{\text {reg }}$ of $G_{\mathbb{C}}$. Let

$$
p_{ \pm}: G_{\mathbb{C}}^{\mathrm{reg}} \longrightarrow \mathfrak{n}_{ \pm}, \quad p_{o}: G_{\mathbb{C}}^{\mathrm{reg}} \rightarrow L_{\mathbb{C}},
$$

be the projections characterized by the identity

$$
\exp \left(p_{-}(g)\right) p_{o}(g) \exp \left(p_{+}(g)\right)=g .
$$


We set

eqn :alpha

eqn : beta

eqn: gamma

$$
\begin{aligned}
& \alpha: \mathfrak{g} \times \mathfrak{n}_{-} \rightarrow \mathfrak{l}, \\
& \beta: \mathfrak{g} \times \mathfrak{n}_{-} \rightarrow \mathfrak{n}_{-}, \\
& \gamma: \mathfrak{g} \times \mathfrak{n}_{-} \rightarrow \mathfrak{l}+\mathfrak{n}_{+},
\end{aligned}
$$$$
\left.(Y, Z) \mapsto \frac{d}{d t}\right|_{t=0} p_{o}\left(e^{t Y} e^{Z}\right)
$$$$
\left.(Y, Z) \mapsto \frac{d}{d t}\right|_{t=0} p_{-}\left(e^{t Y} e^{Z}\right) .
$$$$
(Y, Z) \mapsto \alpha(Y, Z)+\left.\frac{d}{d t}\right|_{t=0} p_{+}\left(e^{t Y} e^{Z}\right) .
$$

We regard $\beta(Y, \cdot)$ as a holomorphic vector field on $\mathfrak{n}_{-}$through the following identification.

$$
\mathfrak{n}_{-} \ni Z \mapsto \beta(Y, Z) \in \mathfrak{n}_{-} \simeq T_{Z} \mathfrak{n}_{-} .
$$

Example 3.7. $G_{\mathbb{C}}=G L(p+q, \mathbb{C}), L_{\mathbb{C}}=G L(p, \mathbb{C}) \times G L(q, \mathbb{C})$, and $\mathfrak{n}_{-} \simeq M(p, q ; \mathbb{C})$.

We note that $\mathfrak{n}_{-}$is realized as upper block matrices. Then for $g^{-1}=\left(\begin{array}{ll}a & b \\ c & d\end{array}\right) \in G_{\mathbb{C}}$, $Y=\left(\begin{array}{ll}A & B \\ C & D\end{array}\right) \in M(p+q ; \mathbb{C})$ and $Z \in M(p, q ; \mathbb{C})$ we have

$$
\begin{array}{ll}
p_{-}\left(g^{-1}\right)=b d^{-1}, & \\
p_{o}\left(g^{-1}\right)=\left(a-b d^{-1} c, d\right) & \in G L(p, \mathbb{C}) \times G L(q, \mathbb{C}), \\
\alpha(Y, Z)=(A-Z C, C Z+D) & \in \mathfrak{g l}_{p}(\mathbb{C}) \oplus \mathfrak{g l}_{q}(\mathbb{C}), \\
\beta(Y, Z) & =A Z+B-Z C Z-Z D .
\end{array}
$$

Then $\beta(Y, \cdot)$ is regarded as the following holomorphic vector field on $\mathfrak{n}_{-} \simeq M(p, q ; \mathbb{C})$ given by

$$
\begin{aligned}
\operatorname{Trace}\left(\beta(Y, Z){ }^{t} \partial_{Z}\right) & =\sum_{a=1}^{p} \sum_{b=1}^{q} \beta(Y, Z)_{a b} \frac{\partial}{\partial z_{a b}} \\
& =\sum_{a=1}^{p} \sum_{b=1}^{q}\left(\sum_{i=1}^{p} A_{a i} z_{i b}+B_{a b}-\sum_{i=1}^{p} \sum_{j=1}^{q} z_{a j} C_{j i} z_{i b}-\sum_{j=1}^{q} z_{a j} D_{j b}\right) \frac{\partial}{\partial z_{a b}} .
\end{aligned}
$$

A reductive Lie algebra $\mathfrak{g}$ is said to be $k$-graded if it admits a direct sum decomposition $\mathfrak{g}=\oplus_{j=-k}^{k} \mathfrak{g}(j)$ such that $[\mathfrak{g}(i), \mathfrak{g}(j)] \subset \mathfrak{g}(i+j)$ for all $i, j$. Any parabolic subalgebra $\mathfrak{p}=\mathfrak{l}+\mathfrak{n}_{+}$of $\mathfrak{g}$ is given by $\mathfrak{l}=\mathfrak{g}(0)$ and $\mathfrak{n}_{+}=\oplus_{j>0} \mathfrak{g}(j)$ for some $k$-gradation of $\mathfrak{g}$. We then have the following estimates of coefficients of holomorphic differential operators $d \pi_{\mu}(Y)$.

Lemma 3.8. According to the direct sum decomposition $\mathfrak{g}=\oplus_{j=-k}^{k} \mathfrak{g}(j)$, we write $\gamma(Y, Z)=\sum_{\ell=0}^{k} \gamma_{\ell}$ and $\beta(Y, Z)=\sum_{\ell=-k}^{-1} \beta_{\ell}$, where $\gamma_{\ell} \in \mathfrak{g}(\ell)$ for $0 \leq \ell \leq k$ and $\beta_{\ell} \in \mathfrak{g}(\ell)$ for $-k \leq \ell \leq-1$. Then $\gamma_{\ell}$ and $\beta_{\ell}$ are polynomials in $Z$ of degree at most $k-\ell$. 
Proof. Since the map $N_{-, \mathbb{C}} \times P_{\mathbb{C}} \stackrel{\sim}{\longrightarrow} G_{\mathbb{C}}^{\text {reg }}$ is an analytic diffeomorphism, we have eqn : tYZ

$$
e^{t Y} e^{Z}=e^{Z+t \beta(Y, Z)+o(t)} e^{t \alpha(Y, Z)+o(t)}
$$

for sufficiently small $t \in \mathbb{C}$, where we use the Landau symbol $o(t)$ for a $\mathfrak{g}$-valued function dominated by $t$ when $t$ tends to be zero. Multiplying (3.10) by $e^{-Z}$ from the left, and taking the differential at $t=0$, we get

$$
\operatorname{Ad}\left(e^{-Z}\right) Y=\gamma(Y, Z)+\frac{e^{\operatorname{ad}(Z)}-1}{\operatorname{ad}(Z)} \beta(Y, Z),
$$

because $\left.\frac{d}{d t}\right|_{t=0} e^{-Z} e^{Z+t W}=\frac{e^{\operatorname{ad}(Z)}-1}{\operatorname{ad}(Z)} W$. We note that $\operatorname{ad}(Z)$ lowers the grading of $\mathfrak{g}$, namely $\operatorname{ad}(Z) \mathfrak{g}(j) \subset \oplus_{i=-k}^{j-1} \mathfrak{g}(i)$ because $Z \in \mathfrak{n}_{-}$. In particular, we have

eqn : YZ

$$
\sum_{j=0}^{2 k} \frac{(-1)^{j}}{j !} \operatorname{ad}(Z)^{j} Y=\gamma(Y, Z)+\sum_{i=0}^{k-1} \frac{\operatorname{ad}(Z)^{i}}{(i+1) !} \beta(Y, Z) .
$$

Let $q_{\ell}: \mathfrak{g} \rightarrow \mathfrak{g}(\ell)$ be the projection according to the direct sum decomposition $\mathfrak{g}=$ $\bigoplus_{j=-k}^{k} \mathfrak{g}(j)$. Suppose $\ell \geq 0$. Applying $q_{\ell}$ to $\frac{\text { and } \cdot \mathrm{YZ}}{3.11}$, we have

$$
\gamma_{\ell}=q_{\ell}\left(\sum_{j=0}^{k-\ell} \frac{(-1)^{j} \mathrm{ad}(Z)^{j}}{j !} Y\right)
$$

Hence $\gamma_{\ell}$ is a polynomial in $Z$ of degreg at most $k-\ell$.

Suppose $\ell<0$. Applying $q_{\ell}$ to (3.11), we get

$$
\beta_{\ell}=q_{\ell}\left(\sum_{j=0}^{k-\ell} \frac{(-1)^{j} \operatorname{ad}(Z)^{j}}{j !} Y\right)-q_{\ell}\left(\sum_{i=0}^{j-\ell} \sum_{j=\ell}^{-1} \frac{\operatorname{ad}(Z)^{i}}{(i+1) !} \beta_{j}\right) .
$$

By the downward induction on $\ell$, we see that $\beta_{\ell}$ is a polynomial in $Z$ of degree at subsec: 3.4 most $k-\ell$ for $-k \leq \ell \leq-1$.

3.3. Fourier transform of principal series representations. Suppose $\mathfrak{g}$ is a complex reductive Lie algebra, $\mathfrak{p}=\mathfrak{l}+\mathfrak{n}_{+}$a parabolic subalgebra, and $\lambda: \mathfrak{p} \rightarrow \operatorname{End}_{\mathbb{C}}(V)$ a finite-dimensional representation.

We use the letter $\mu$ to denote the representation of $\mathfrak{p}$ on the dual space $V^{\vee}$ given by

eqn: mucompl

$$
\mu:=\lambda^{*} \equiv \lambda^{\vee} \otimes \operatorname{Trace}\left(\operatorname{ad}(\cdot): \mathfrak{n}_{+} \rightarrow \mathfrak{n}_{+}\right) .
$$

By applying the (algebraic) Fourier transform of the Weyl algebra, we define a Lie algebra homomorphism

$$
\widehat{d \pi_{\mu}}: \mathfrak{g} \rightarrow \mathcal{D}\left(\mathfrak{n}_{+}\right) \otimes \operatorname{End}_{\mathbb{C}}\left(V^{\vee}\right),
$$


by using the complex flag variety $G_{C} / P_{\mathbb{C}}$ in this subsection. In Section 3 sec: 33 part 1 $\widehat{d \pi_{\mu}}$ with the "algebraic Fourier transform" of a generalized Verma module

$$
F_{c}: \operatorname{ind}_{\mathfrak{p}}^{\mathfrak{g}}\left(V^{\vee}\right) \stackrel{\sim}{\rightarrow} \operatorname{Pol}\left(\mathfrak{n}_{+}\right) \otimes V^{\vee},
$$

which is defined by using a real flag variety $G / P$, see $\left(\frac{\text { eqn } \cdot i n d P .23)}{3.2}\right.$.

Let $G_{\mathbb{C}}$ be a connected complex reductive Lie group with complex reductive Lie algebra $\mathfrak{g}$, and $P_{\mathbb{C}}=L_{\mathbb{C}} N_{+, \mathbb{C}}$ be the parabolic subgroup with Lie algebra $\mathfrak{p}$. Let $\Omega_{X_{\mathbb{C}}}$ be the canonical line bundle of the complex generalized flag variety $X_{\mathbb{C}}=G_{\mathbb{C}} / P_{\mathbb{C}}$.

Suppose $\lambda$ lifts to a holomorphic representation of $P_{\mathbb{C}}$, then so does $\mu$. We form a

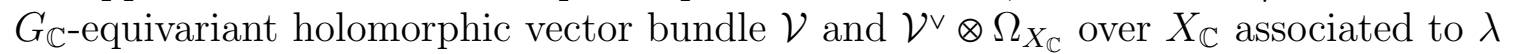
and $\mu$, respectively.

We consider the regular representation $\pi_{\mu}$ of $G_{\mathbb{C}}$ on $C^{\infty}\left(G_{\mathbb{C}} / P_{\mathbb{C}}, \mathcal{V}^{\vee} \otimes \Omega_{X_{\mathbb{C}}}\right)$. The infinitesimal action will be denoted by $d \pi_{\mu}$, which is defined on $C^{\infty}\left(U,\left.\mathcal{V}^{\vee} \otimes \Omega_{X_{\mathbb{C}}}\right|_{U}\right)$ for any open subset $U$ of $G_{\mathbb{C}} / P_{\mathbb{C}}$. In particular, we take $U$ to be the open Bruhat cell $\mathfrak{n}_{-} \hookrightarrow G_{\mathbb{C}} / P_{\mathbb{C}}, Z \mapsto \exp Z \cdot o$, where $o=e P_{\mathbb{C}} \in G_{\mathbb{C}} / P_{\mathbb{C}}$. By trivializing the holomorphic vector bundle $\mathcal{V}^{\vee} \otimes \Omega_{X_{\mathbb{C}}} \rightarrow G_{\mathbb{C}} / P_{\mathbb{C}}$ on it, we define a function $F \in C^{\infty}\left(\mathfrak{n}_{-}, V^{\vee}\right)$ for a section $f \in C^{\infty}\left(G_{\mathbb{C}} / P_{\mathbb{C}}, \mathcal{V}^{\vee} \otimes \Omega_{X_{\mathbb{C}}}\right)$ by

$$
F(Z):=f(\exp Z) \quad \text { for } Z \in \mathfrak{n}_{-} .
$$

Then the action of $\mathfrak{g}$ on $C^{\infty}\left(\mathfrak{n}_{-}, V^{\vee}\right)$ given by

eqn :351

$$
\begin{aligned}
\left(d \pi_{\mu}(Y) F\right)(Z)= & =\left.\frac{d}{d t}\right|_{t=0} f\left(e^{-t Y} e^{Z}\right) \\
& =\mu(\gamma(Y, Z)) F(Z)-(\beta(Y, \cdot) F)(Z) \quad \text { for } Y \in \mathfrak{g},
\end{aligned}
$$

where by a little abysse of notation $\mu$ stands for the infinitesimal action. The righthand side of (3.13) defines a representation of Lie algebra $\mathfrak{g}$ whenever $\mu$ (or $\lambda$ ) is a representation of the Lie algebra $\mathfrak{p}$ without assuming that it lifts to a holomorphic representation of the complex reductive group $P_{\mathbb{C}}$.

It follows from (3.13) and Lemma that we obtain a Lie algebra homomorphism

eqn:dpi1

$$
d \pi_{\mu}: \mathfrak{g} \rightarrow \mathcal{D}\left(\mathfrak{n}_{-}\right) \otimes \operatorname{End}\left(V^{\vee}\right),
$$

for any representation $\lambda$ of the Lie algebra $\mathfrak{p}$. By taking the algebraic Fourier transform on the Weyl algebra $\mathcal{D}\left(\mathfrak{n}_{-}\right)$(see Definition 3.1], we get another Lie algebra homomorphism:

$$
\widehat{d \pi_{\mu}}: \mathfrak{g} \rightarrow \mathcal{D}\left(\mathfrak{n}_{+}\right) \otimes \operatorname{End}\left(V^{\vee}\right)
$$

We use the same letter $\pi_{\mu}$ to denote the "action" of $G_{\mathbb{C}}$ on $C^{\infty}\left(\mathfrak{n}_{-}, V^{\vee}\right)$ given as eqn: gNpic

$$
\left(\pi_{\mu}(g) F\right)(Z)=\mu\left(p_{o}\left(g^{-1} \exp Z\right) \exp \left(p_{+}\left(g^{-1} \exp Z\right)\right)\right)^{-1} F\left(p_{-}\left(g^{-1} \exp Z\right)\right) .
$$


This formula makes sense if $F$ comes from $C^{\infty}\left(G_{\mathbb{C}} / P_{\mathbb{C}}, \mathcal{V} \otimes \Omega_{X_{\mathbb{C}}}^{\vee}\right)$, or if $F \in C^{\infty}\left(\mathfrak{n}_{-}, V^{\vee}\right)$ and $g \in G_{\mathbb{C}}$ and $Z \in \mathfrak{n}_{-}$satisfy $g^{-1} \exp Z \in G_{\mathbb{C}}^{\text {reg }}$. In particular, if $\lambda$ is trivial on the nilpotent radical $\mathfrak{n}_{+}$for $g=m \exp W$ with $m \in L_{\mathbb{C}}$ and $W \in \mathfrak{n}_{-}$, and if $\mathfrak{n}_{+}$is abelian we have

eqn:simpler

$$
\left(\pi_{\mu}(g) F\right)(Z)=\mu(m) F\left(\operatorname{Ad}(m)^{-1} Z-W\right) .
$$

Let us analyze $d \pi_{\mu}(Y)$ for $Y \in \mathfrak{l}+\mathfrak{n}_{+}$. We begin with the case $Y \in \mathfrak{l}$. We let the Levi subgroup $L_{\mathbb{C}}$ act on $\operatorname{Pol}\left(\mathfrak{n}_{+}\right)$by

$$
\operatorname{Ad}_{\#}(l): f(\cdot) \mapsto f\left(\operatorname{Ad}\left(l^{-1}\right) \cdot\right), \quad l \in L_{\mathbb{C}} .
$$

Since this action is algebraic, the infinitesimal action defines a Lie algebra homomorphism into the Weyl algebra:

$$
\operatorname{ad}_{\#}: \mathfrak{l} \rightarrow \mathcal{D}\left(\mathfrak{n}_{+}\right), \quad Y \mapsto \operatorname{ad}_{\#}(Y),
$$

where $\operatorname{ad}_{\#}(Y)$ is a holomorphic vector field on $\mathfrak{n}_{+} \operatorname{given}$ by $\operatorname{ad}_{\#}(Y)_{x}:=\left.\frac{d}{d t}\right|_{t=0} \operatorname{Ad}\left(e^{-t Y}\right) x \in$ $T_{x}\left(\mathfrak{n}_{+}\right)$for $x \in \mathfrak{n}_{+}$.

lem:410 Lemma 3.9. Let $\lambda$ be a representation of the parabolic Lie algebra $\mathfrak{p}=\mathfrak{l}+\mathfrak{n}_{+}$, and $\mu \equiv \lambda^{*}$ be as in $(3.12)$. Then the following two representations of $\mathfrak{l}$ on $\operatorname{Pol}\left(\mathfrak{n}_{+}\right) \otimes V^{\vee}$ are isomorphic:

$$
\left.\widehat{d \pi_{\mu}}\right|_{\mathfrak{l}} \simeq \operatorname{ad}_{\#} \otimes \mathrm{id}+\mathrm{id} \otimes\left(\mu-\text { Trace }\left.\circ \mathrm{ad}\right|_{\mathfrak{n}-}\right)=\operatorname{ad}_{\#}(Y) \otimes \mathrm{id}+\mathrm{id} \otimes(-\lambda) .
$$

In particular, if $\lambda$ lifts to a holomorphic representation of $P_{\mathbb{C}}$ then the right-hand side is the infinitesimal action of $\operatorname{Ad}_{\#} \otimes \lambda^{\vee}$ of $L_{\mathbb{C}}$ on $\operatorname{Pol}\left(\mathfrak{n}_{+}\right) \otimes V^{\vee}$.

Proof. For $Y \in \mathfrak{l}, X \in \mathfrak{n}_{-}$we have $\gamma(Y, X)=Y$, and the formula 3.1351 reduces, in $\mathcal{D}\left(\mathfrak{n}_{-}\right) \otimes \operatorname{End}\left(V^{\vee}\right)$, to

$$
d \pi_{\mu}(Y)=\mathrm{id} \otimes \mu(Y)-\beta(Y, \cdot) \otimes \mathrm{id} .
$$

We apply Lemma 3.4 to the case where $(\sigma, E)$ is the adjoint representation of $\mathfrak{l}$ on $\mathfrak{n}_{-}$. Since $\beta(Y, \cdot)=-d L(Y)$ for $Y \in \mathfrak{l}$, we have $\Psi_{\mathfrak{n}_{-}} \circ$ ad $=-\beta$ on $\mathfrak{l}$, with the notation therein. Moreover, via the identification $\mathfrak{n}_{-}^{\vee} \simeq \mathfrak{n}_{+}$, the map $\Psi_{\mathfrak{n} \vee} \circ$ ad ${ }^{\vee}$ amounts to $\Psi_{\mathfrak{n}_{+}} \circ$ ad $=\operatorname{ad}_{\#}$. Therefore, we get

$$
\begin{aligned}
\widehat{d \pi_{\mu}}(Y) & =\mathrm{id} \otimes \mu(Y)+\Psi_{\mathfrak{n}_{-}} \circ \operatorname{ad}^{\vee}(Y) \otimes \mathrm{id}+\left(\left.\operatorname{Trace} \circ \operatorname{ad}(Y)\right|_{\mathfrak{n}_{-}}\right) \mathrm{id} \otimes \mathrm{id} \\
& =\mathrm{id} \otimes \mu(Y)+\operatorname{ad}_{\#}(Y) \otimes \mathrm{id}-\left(\left.\operatorname{Trace} \circ \operatorname{ad}(Y)\right|_{\mathfrak{n}_{+}}\right) \mathrm{id} \otimes \mathrm{id} .
\end{aligned}
$$

Thus, the lemma follows. 
The differential operators $\widehat{d \pi_{\mu}}(Y)$ with $Y \in \mathfrak{n}_{+}$play a central role in the F-method. If the parabolic subalgebra $\mathfrak{p}$ is associated to a $k$-gradation of $\mathfrak{g}$, then these differential operators are at most of order $2 k$ by Lemma 3.8. We describe their structure in the case where $k=1$, namely $\mathfrak{n}_{+}$is abelian.

lem:315 Proposition 3.10. Assume that $\mathfrak{n}_{+}$is abelian Let $\left(\lambda_{,}, V\right)$ be a representation of $\mathfrak{l}$, extended trivially on $\mathfrak{n}_{+}$, and $\mu=\lambda^{*}$ be as in 3.12 . For every $Y \in \mathfrak{n}_{+}$the operator $\widehat{d \pi_{\mu}}(Y)$ is of the form

eqn : degree

$$
\sum a_{i}^{j k} \zeta^{i} \frac{\partial^{2}}{\partial \zeta^{j} \partial \zeta^{k}}+\sum b^{j} \frac{\partial}{\partial \zeta^{j}}
$$

where $a_{i}^{j k}$ and $b^{j} \in \operatorname{End}\left(V^{\vee}\right)$ are constants depending on $Y$.

Proof. Since $\mathfrak{n}_{+}$is abelian, we can take a characteristic element $H$ such that

$$
\operatorname{Ad}\left(e^{s H}\right) Y=e^{s} Y \quad \text { for any } Y \in \mathfrak{n}_{+} .
$$

We set $m:=e^{s H}$. Then ${ }^{t} \operatorname{Ad}(m)^{-1}=e^{-s}$ id on $\mathfrak{n}_{-} \simeq \mathfrak{n}_{+}^{\vee}$.

Taking the algebraic Fourier transform of the formula

$$
d \pi_{\mu}(\operatorname{Ad}(m) Y)=\pi_{\mu}(m) d \pi_{\mu}(Y) \pi_{\mu}\left(m^{-1}\right),
$$

where $\left(\pi_{\mu}(m) F\right)(Z)=\mu(m) F\left(\operatorname{Ad}\left(m^{-1}\right) Z\right)=\mu(m) F\left(\operatorname{Ad}(m)_{\#} F\right)(Z)$ by $\left.\frac{\text { leqn } \cdot \text { simpler }}{3.17}\right)$, we get

by Lemma 3.3. Hence

$$
\widehat{d \pi_{\mu}}(\operatorname{Ad}(m) Y)=\left({ }^{t} \operatorname{Ad}(m)^{-1}\right)_{*} \widehat{d \pi_{\mu}}(Y)
$$

eqn : Rt

$$
e^{s} \widehat{d \pi_{\mu}}(Y)=\left(e^{-s} \mathrm{id}\right)_{*} \widehat{d \pi_{\mu}}(Y)
$$

If we write $\widehat{d \pi_{\mu}}(Y)$ in the form

$$
\sum_{\alpha, \beta \in \mathbb{N} n} C_{\alpha, \beta} \zeta^{\alpha} \frac{\partial^{|\beta|}}{\partial \zeta^{\beta}}
$$

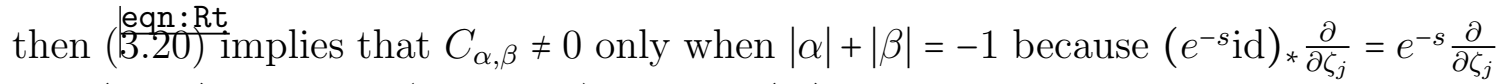
and $\left(e^{-s} \mathrm{id}\right)_{*} \zeta_{j}=e^{s} \zeta_{j}(1 \leq j \leq n)$. As $d \pi_{\mu d}(Y)$ is a vector field there is no term for $|\alpha|>1$. Hence we get the expression (3.19).

3.4. Fourier transform on the real flag varieties. In this subsection we define "algebraic Fourier transform" of generalized Verma modules, see (3.23):

$$
F_{c}: \operatorname{ind}_{\mathfrak{p}}^{\mathfrak{g}}\left(V^{\vee}\right) \stackrel{\sim}{\rightarrow} \operatorname{Pol}\left(\mathfrak{n}_{+}\right) \otimes V^{\vee} .
$$


As we shall prove in Proposition $3.11: F c$ the Lie algebra homomorphism $\widehat{d \pi_{\lambda^{*}}}: U(\mathfrak{g}) \longrightarrow$ $\mathcal{D}\left(\mathfrak{n}_{+}\right) \otimes \operatorname{End}\left(V^{\vee}\right)$ defined in 3.15 in the previous section can be reconstructed from $F_{c}$, namely, $\widehat{d \pi_{\lambda^{*}}}(u)(u \in U(\mathfrak{g}))$ is the operator $S$ that is characterized by

$$
S F_{c}(v)=F_{c}(u \cdot v) \quad \text { for any } v \in \operatorname{ind}_{\mathfrak{p}}^{\mathfrak{g}}\left(V^{\vee}\right) .
$$

For later purpose, we work with a real form $G$ of $G_{\mathbb{C}}$. From now on, let $G$ be a real semisimple Lie group, $P$ a parabolic subgroup of $G$ with Levi decomposition $P=L N_{+}$, and $V$ a finite-dimensional representation of $P$.

Let $L N_{-}$be the opposite parabolic subgroup of $P=L N_{+}$. We write $\mathfrak{n}_{+}(\mathbb{R})$ and $\mathfrak{n}_{-}(\mathbb{R})$ for the Lie algebras of $N_{+}$and $N_{-}$, respectively, and set $\mathfrak{n}_{+}=\mathfrak{n}_{+}(\mathbb{R}) \otimes_{\mathbb{R}} \mathbb{C}$. The open Bruhat cell is given as the image of the following embedding

$$
\iota: \mathfrak{n}_{-}(\mathbb{R}) \hookrightarrow G / P, \quad X \mapsto \exp (X) \cdot o,
$$

where $o=e P \in G / P$.

Let $\lambda: P \rightarrow G L_{\mathbb{C}}(V)$ be a finite-dimensional representation of $P$, and $\mathcal{V}=G \times_{P} V$ the $G$-equivariant vector bundle over the real flag variety $G / P$. The pullback of the dualizing bundle $\mathcal{V}^{*} \equiv \mathcal{V}_{2 \rho}^{\vee} \rightarrow G / P$ via $\iota$ is trivialized into the direct product bundle $\mathfrak{n}_{-}(\mathbb{R}) \times V^{\vee} \rightarrow \mathfrak{n}_{-}(\mathbb{R})$ and thus we have a linear isomorphism:

eqn:iso1

$$
\iota^{*}: \mathcal{E}_{\{o\}}^{\prime}\left(G / P, \mathcal{V}_{2 \rho}^{\vee}\right) \stackrel{\sim}{\longrightarrow} \mathcal{E}_{\{0\}}^{\prime}\left(\mathfrak{n}_{-}(\mathbb{R})\right) \otimes V^{\vee}
$$

through which we induce the $(\mathfrak{g}, P)$-action on $\mathcal{E}_{\{0\}}^{\prime}\left(\mathfrak{n}_{-}(\mathbb{R})\right) \otimes V^{\vee}$ from $\mathcal{E}_{\{o\}}^{\prime}\left(G / P, \mathcal{V}_{2 \rho}^{\vee}\right)$.

The Killing form of $\mathfrak{g}$ identifies the dual space $\mathfrak{n}_{-}(\mathbb{R})^{\vee}$ with $\mathfrak{n}_{+}(\mathbb{R})$, and thus the Fourier transform $\mathcal{F}_{c}$ in 3.4 gives rise to a linear isomorphism:

eqn: iso11

$$
\mathcal{F}_{c} \otimes \mathrm{id}: \mathcal{E}_{\{0\}}^{\prime}\left(\mathfrak{n}_{-}(\mathbb{R})\right) \otimes V^{\vee} \stackrel{\sim}{\longrightarrow} \operatorname{Pol}\left(\mathfrak{n}_{+}\right) \otimes V^{\vee},
$$

through which we induce the $(\mathfrak{g}, P)$-action further on the right-hand side.

In summary we have the following $(\mathfrak{g}, P)$-isomorphisms:

$$
F_{c}: \operatorname{ind}_{\mathfrak{p}}\left(V^{\vee}\right) \stackrel{\substack{\text { eqn } \\ \stackrel{(2.22)}{\sim}}}{\longrightarrow} \mathcal{E}_{\{o\}}^{\prime}\left(G / P, \mathcal{V}_{2 \rho}^{\vee}\right) \stackrel{\substack{\text { eqn } \\ \stackrel{(3.21)}{\sim}}}{\longrightarrow} \mathcal{E}_{\{0\}}^{\prime}\left(\mathfrak{n}_{-}(\mathbb{R})\right) \otimes V^{\vee} \stackrel{\mathcal{F}_{c} \otimes \text { id }}{\longrightarrow} \operatorname{Pol}\left(\mathfrak{n}_{+}\right) \otimes V^{\vee} .
$$

We say that $F_{c}$ is the algebraic Fourier transform of a generalized Verma module.

The $(\mathfrak{g}, P)$-module structure of $\operatorname{Pol}\left(\mathfrak{n}_{+}\right) \otimes V^{\vee}$ is described by the following proposition.

prop:Fc Proposition 3.11. Let $(\lambda, V)$ be a finite-dimensional representation of $P$ and define another representation of $P$ on the dual space $V^{\vee}$ by $\mu:=\lambda^{*} \equiv \lambda^{\vee} \otimes \mathbb{C}_{2 \rho}$. Then,

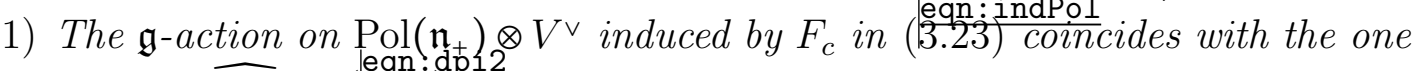
given by $\widehat{d \pi_{\mu}}$ in

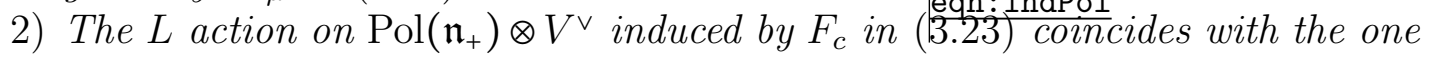
given by $\mathrm{Ad}_{\#} \otimes \lambda^{\vee}$. 
Proof. 1) Let $G_{\mathbb{C}}$ be a complexification of $G$ and $P_{\mathbb{C}}$ the connected subgroup of $G_{\mathbb{C}}$ with Lie algebra $\mathfrak{p}=\operatorname{Lie}(P) \otimes_{\mathbb{R}} \mathbb{C}$. First we assume that $\lambda$ extends to a holomorphic representation of $P_{\mathbb{C}}$. Then the $G$-equivariant vector bundle $\mathcal{V}_{2 \rho}^{\vee}$ over $X=G / P$ is the restriction of the $G_{\mathbb{C}^{-}}$equivariant holomorphic vector bundle $\mathcal{V}^{\vee} \otimes \Omega_{X_{\mathbb{C}}}$ over $X_{\mathbb{C}}=G_{\mathbb{C}} / P_{\mathbb{C}}$ that was introduced in the previous subsection. Therefore, the action of $Y \in \mathfrak{g}$ on $\mathcal{E}_{\{0\}}^{\prime}\left(\mathfrak{n}_{-}(\mathbb{R}) \otimes V^{\vee}\right)$ induced by $\iota^{*}$ in $[3.21)$ is given by the restriction of the holomorphic differential operator $d \pi_{\mu}(Y)$.

In turn, the action of $Y \in \mathfrak{g}$ on $\operatorname{Pol}\left(\mathfrak{n}_{+}\right) \otimes V^{\vee}$ induced by the isomorphism $\left(\frac{\text { eqn.iso11 }}{3.22} \mid\right.$ is given by

$$
\left(\mathcal{F}_{c} \otimes \mathrm{id}\right) \circ d \pi_{\mu}(Y) \circ\left(\mathcal{F}_{c}^{-1} \otimes \mathrm{id}\right),
$$

which is equal to $\overline{d \pi_{\mu}(Y)}$ by Remark

To complete the proof in the general case we denote by $\operatorname{Hom}\left(P_{\mathbb{C}}, G L_{\mathbb{C}}(V)\right)$ the set of holomorphic representations of $P_{\mathbb{C}}$ on $V$ and by $\operatorname{Hom}(\mathfrak{p}, \operatorname{End}(V))$ the set of Lie algebra representations of $\mathfrak{p}$. Since the former is Zariski dense in the latter, the two $\mathfrak{g}$-actions on $\operatorname{Pol}\left(\mathfrak{n}_{+}\right) \otimes V^{\vee}$ coincide for all $\lambda$ because both depend algebraically (actually affinely) on $\lambda \in \operatorname{Hom}(\mathfrak{p}, \operatorname{End}(V))$.

2) This statement is the analogue of Lemma for the Lie group $L$. Indeed, since the group $L$ normalizes $\mathfrak{n}_{-}(\mathbb{R})$ and fixes the origin 0 , the isomorphism $\iota^{*}$ in $\left(\frac{e a n}{3.21}\right)$ respects the $L$-action when $L$ acts diagonally on $\mathcal{E}_{\{0\}}^{\prime}\left(\mathfrak{n}_{-}(\mathbb{R})\right) \otimes V^{\vee}$. To conclude the proof we use (3.5).

The map $F_{c}$ does not depend on the choice of a real form $G$ of $G_{\mathbb{C}}$ that appears in the two middle terms of $(3.23)$. Moreover, the isomorphism $F_{c}: \operatorname{ind}_{\mathfrak{p}}^{\mathfrak{g}}\left(V^{\vee}\right) \stackrel{\sim}{\longrightarrow}$ $\operatorname{Pol}\left(\mathfrak{n}_{+}\right) \otimes V^{\vee}$ depends only on the infinitesimal action of $P$ on $V$. In fact the following corollary follows immediately from the statement 1) of Proposition 3.11 .

cor:Fc Corollary 3.12. The algebraic Fourier transform of generalized Verma modules (see (3.23)

$$
F_{c}: \operatorname{ind}_{\mathfrak{p}}^{\mathfrak{g}}\left(V^{\vee}\right) \stackrel{\sim}{\longrightarrow} \operatorname{Pol}\left(\mathfrak{n}_{+}\right) \otimes V^{\vee}
$$

is given by

$$
\left.\left(u \otimes v^{\vee}\right) \mapsto \widehat{d \pi_{\lambda^{*}}(u}\right)\left(1 \otimes v^{\vee}\right), \quad u \in U(\mathfrak{g}), v^{\vee} \in V^{\vee}
$$

\section{F-METHOD}

In Section 2 we have established a one-to-one correspondence between differential symmetry breaking operators for vector bundles and certain Lie algebra homomorphisms (Theorem 2.9). Using this framework our aim is to find explicit formulæ for such operators, in particular, when such operators are a priori known to be unique up to scalar. For this purpose we propose a new method, which we call the F-method. 
Its theoretical foundation is summarized in Theorem $\frac{\mid t h m: 313}{4.1}$ This method becomes particularly simple when $\mathfrak{h}$ is a parabolic subalgebra with abelian nilradical. In this case we develop the F-method in more details, and give its recipe in Section 4.4 . Some useful lemmas for actual computations for vector-valued differential operators are collected in Section 4.5 .

\subsection{Construction of equivariant differential operators by algebraic Fourier} transform. Let $E$ be a finite-dimensional vector spaces over $\mathbb{C}$ and $E^{\vee}$ its dual space. Let Diff ${ }^{\text {const }}(E)$ denote the ring of holomorphic differential operators on $E$ with constant coefficients. We define the symbol map

$$
\text { Symb : Diff }{ }^{\text {const }}(E) \stackrel{\sim}{\longrightarrow} \operatorname{Pol}\left(E^{\vee}\right), \quad D_{z} \mapsto Q(\zeta)
$$

by the following characterization

$$
D_{z} e^{\langle z, \zeta\rangle}=Q(\zeta) e^{\langle z, \zeta\rangle}
$$

Then Symb is an algebra isomorphism. The differential operator on $E$ with symbol $Q(\zeta)$ will be denoted by $\partial Q_{z}$.

By the definition of the algebraic Fourier transform (Definition 3.1 ) one has

eqn : aFP

$$
\widehat{\partial P_{z}}=(-1)^{\ell} P(\zeta), \quad \widehat{Q(z)}=\partial Q_{\zeta}
$$

for any homogeneous polynomial $P$ on $E^{\vee}$ of degree $\ell$ and any polynomial $Q$ on $E$ seen as a multiplication operator

We recall from Corollary 3.12 that $\operatorname{Pol}\left(\mathfrak{n}_{+}\right) \otimes V^{\vee}$ is a $(\mathfrak{g}, P)$-module if $V$ is a $P$ module. Note that the action of $\exp \left(\mathfrak{n}_{+}\right)(\subset P)$ on $\operatorname{Pol}\left(\mathfrak{n}_{+}\right) \otimes V^{\vee}$ is not geometric, namely, it is not given by the pull-back of polynomials via the action on the base space $\mathfrak{n}_{+}$.

The key tool for the F-method that we explain in Section 4.4 is the following 44 ilinear assertion. We note that the two approachess (łhe canonical invariant pairing (2.20)) and the algebraic Fourier transform (3.23) give rise to the same differential operators, provided that $\mathfrak{n}_{+}$is abelian:

thm:313 Theorem 4.1. Suppose that $\mathfrak{p}$ is a parabolic subalgebra $\mathfrak{g}$ and that $P=L \exp \left(\mathfrak{n}_{+}\right)$ is its Levi decomposition. Let $P^{\prime}$ be a closed subgroup of $P$ such that $P^{\prime}$ has a decomposition $P^{\prime}=L^{\prime} \exp \left(\mathfrak{n}_{+}^{\prime}\right)$ with $L^{\prime} \subset L$ and $\mathfrak{n}_{+}^{\prime} \subset \mathfrak{n}_{+}$. Let $G^{\prime}$ be an arbitrary subgroup of $G$ containing $P^{\prime}$. For a representation $(\lambda, V)$ of $P$ and a representation $(\nu, W)$ of $P^{\prime}$, we form a $G$-equivariant vector bundle $\mathcal{V}=G \times{ }_{P} V$ over $X=G / P$ and $a$ $G^{\prime}$-equivariant vector bundle $\mathcal{W}=G^{\prime} \times_{P^{\prime}} W$ over $Y=G^{\prime} / P^{\prime}$, respectively. Let $\mu:=\lambda^{*}$ be as in (3.12). 
(1) There is a natural isomorphism

eqn: thm41

n: Fmethod1

n:Fmethod2

$$
\begin{aligned}
\operatorname{Diff}_{G^{\prime}}\left(\mathcal{V}_{X}, \mathcal{W}_{Y}\right) & \simeq\left(\operatorname{Pol}\left(\mathfrak{n}_{+}\right) \otimes \operatorname{Hom}_{\mathbb{C}}(V, W)\right)^{L^{\prime}, \widehat{d \pi_{\mu}}\left(\mathfrak{n}_{+}^{\prime}\right)} \\
& \simeq\left(\operatorname{Hom}_{L^{\prime}}\left(V \otimes \operatorname{Pol}\left(\mathfrak{n}_{+}\right), W\right)\right)^{\widehat{d \pi_{\mu}}\left(\mathfrak{n}_{+}^{\prime}\right)} .
\end{aligned}
$$

Here the right-hand side of $\frac{\text { ean }}{4.2}$ : thm 41 consists of $\psi \in \operatorname{Pol}\left(\mathfrak{n}_{+}\right) \otimes \operatorname{Hom}_{\mathbb{C}}(V, W)$ satisfying

$$
\begin{aligned}
\nu(\ell) \circ \operatorname{Ad}_{\#}(\ell) \psi \circ \lambda\left(\ell^{-1}\right)=\psi & \text { for all } \ell \in L^{\prime}, \\
\left(\widehat{d \pi_{\mu}}(C) \otimes \operatorname{id}_{W}+\mathrm{id} \otimes \nu(C)\right) \psi=0 & \text { for all } C \in \mathfrak{n}_{+}^{\prime} .
\end{aligned}
$$

(2) Assume that the nilradical $\mathfrak{n}_{+}$is abelian. Then the following diagram commutes:

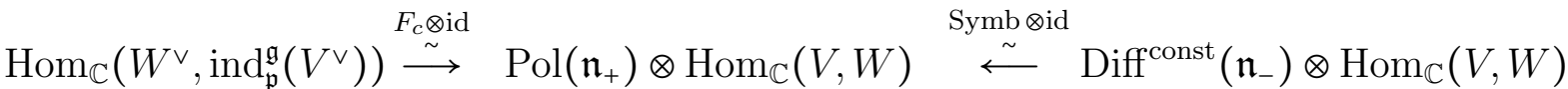

$$
\begin{aligned}
& \cup \\
& \operatorname{Hom}_{P^{\prime}}\left(W^{\vee}, \operatorname{ind}_{\mathfrak{p}}^{\mathfrak{g}}\left(V^{\vee}\right)\right) \\
& \underset{D_{X \rightarrow Y}}{\stackrel{\circlearrowright}{\longrightarrow}} \\
& \operatorname{Diff}_{G^{\prime}}\left(\mathcal{V}_{X}, \mathcal{W}_{Y}\right)
\end{aligned}
$$

Remark 4.2. The convention on the Fourier transform $\mathcal{F}_{c}$ in Definition 3.5 makes the diagram in Theorem 4.1 commutative without additional powers of $\sqrt{-1}$.

Theorem 4 thm 313 may be regarded as a construction of symmetry breaking operators by using the Fourier transform of generalized Verma modules.

Corollary 4.3. Assume that $\mathfrak{n}_{+}$is abelian and that $P^{\prime}=L^{\prime} \exp \left(\mathfrak{n}_{+}^{\prime}\right)$ with $L^{\prime} \subset L$ and $\mathfrak{n}_{+}^{\prime} \subset \mathfrak{n}_{+}$. Then the following diagram of three isomorphisms commutes.

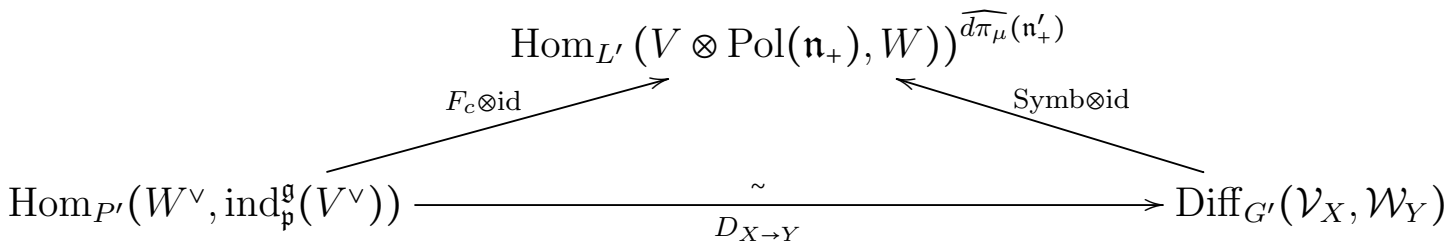

In the above corollary, $\left.\operatorname{Hom}_{L^{\prime}}\left(V \otimes \operatorname{Pol}\left(\mathfrak{n}_{+}\right), W\right)\right)^{\widehat{d \pi_{\mu}}\left(\mathfrak{n}_{+}^{\prime}\right)}$ consists of $L^{\prime}$-equivariant, $\operatorname{Hom}_{\mathbb{C}}(V, W)$-valued polynomial solutions $\psi$ on $\mathfrak{n}_{\text {t }}$ to a system of partial differential equations of second order, see Sections 3.3 and 4.4 . Corollary 4.3 implies that, once we find such a polynomial solution $\psi$, we obtain a $P^{\prime}$-submodule $W^{\vee}$ in $\operatorname{ind}_{\mathfrak{p}}^{\mathfrak{g}}\left(V^{\vee}\right)$ (sometimes referred to as singular vectors) by $\left(F_{c} \otimes \mathrm{id}\right)^{-1}(\psi)$, and a differential symmetry breaking operator by $(\text { Symb } \otimes \mathrm{id})^{-1}(\psi)$.

We first give proofs for the first statement of Theorem 4.1 here. The proof of the second statement is postponed until the next subsection. 
Proof of Theorem 4.1 (1). Combining the duality theorem (Theorem 2.9 ) with the algebraic Fourier transform (Corollary 3.12 ) we have an isomorphism

$$
\operatorname{Hom}_{P^{\prime}}\left(W^{\vee}, \operatorname{Pol}\left(\mathfrak{n}_{+}\right) \otimes V^{\vee}\right) \stackrel{\sim}{\longrightarrow} \operatorname{Diff}_{G^{\prime}}\left(\mathcal{V}_{X}, \mathcal{W}_{Y}\right)
$$

where the $P^{\prime}$-action on $\operatorname{Pol}\left(\mathfrak{n}_{+}\right) \otimes V^{\vee}$ is defined via the algebraic Fourier transform $F_{c}$, namely, the left-hand side consists of $\psi \in \operatorname{Hom}_{\mathbb{C}}\left(W^{\vee}, \operatorname{Pol}\left(\mathfrak{n}_{+}\right) \otimes V^{\vee}\right) \simeq \operatorname{Pol}\left(\mathfrak{n}_{+}\right) \otimes$ $\operatorname{Hom}_{\mathbb{C}}(V, W)$ satisfying

$$
\left(\widehat{d \pi_{\mu}}(C) \otimes \mathrm{id}_{W}+\mathrm{id} \otimes \nu(C)\right) \psi=0 \quad \text { for all } C \in \mathfrak{l}^{\prime}+\mathfrak{n}_{+},
$$

provided $L^{\prime}$ is connected. Owing to Lemma 3.9 , the condition for $C \in \mathfrak{l}^{\prime}$ is equivalent to that $\psi \in\left(\operatorname{Pol}\left(\mathfrak{n}_{+}\right) \otimes \operatorname{Hom}_{\mathbb{C}}(V, W)\right)^{\mathfrak{l}^{\prime}}$, where $\mathfrak{l}^{\prime}$ acts on $\operatorname{Pol}\left(\mathfrak{n}_{+}\right) \otimes \operatorname{Hom}_{\mathbb{C}}(V, W)$ by $\operatorname{ad}_{\#} \otimes \mathrm{id}+\mathrm{id} \otimes\left(\lambda^{\vee} \otimes \mathrm{id}+\mathrm{id} \otimes \nu\right)$.

In a more general setting where we allow $L^{\prime}$ to be disconnected, by the same argument as in the proof of Lemma $\frac{13.9}{3.9}$, we see that the $P$-action on $\operatorname{Pol}\left(\mathfrak{n}_{+}\right) \otimes V^{\vee}$ via the algebraic Fourier transform $F_{c}$ of generalized Verma modules (Corollary 3.12 ) coincides with the tensor product representation $\operatorname{Ad}_{\#} \otimes \lambda^{\vee}$ when restricted to the Levi subgroup $L$. Thus the isomorphism 4.2 is proved.

4.2. Symbol map and reversing signatures. The purpose of this section is to carefully and clearly set up relations involving various signatures in connection with the algebraic Fourier transform in a coordinates-free fashion.

Denote by $\gamma: S(E) \stackrel{\sim}{\rightarrow} \operatorname{Pol}\left(E^{\vee}\right)$ the canonical isomorphism, and define another algebra isomorphism

$$
\gamma_{s g n}: S(E) \stackrel{\sim}{\rightarrow} \operatorname{Pol}\left(E^{\vee}\right)
$$

by $\gamma \circ a$, where $a: S(E) \rightarrow S(E)$ denotes the automorphism of the symmetric algebra $S(E)$ induced by the linear map $X \mapsto-X$ for $X \in E$.

Now we regard $E$ as an abelian Lie algebra over $\mathbb{C}$, and identify its enveloping algebra $U(E)$ with the symmetric algebra $S(E)$. Then, the right and left-infinitesimal actions induce two isomorphisms:

$$
d R: S(E) \stackrel{\sim}{\rightarrow} \operatorname{Diff}^{\text {const }}(E), \quad d L: S(E) \stackrel{\sim}{\rightarrow} \operatorname{Difff}^{\text {const }}(E) .
$$

By the definition of the symbol map, we get,

$$
\text { Symb } \circ d R=\gamma, \quad \text { Symb } \circ d L=\gamma_{s g n} .
$$

On the other hand, it follows from $\frac{\text { legn: }: \text { aFP }}{4.11)}$ that

$$
\overline{d L(u)}=\gamma(u), \quad \overline{d R(u)}=\gamma_{s g n}(u),
$$

for every $u \in S(E) \simeq U(E)$, where polynomials are regarded as multiplication operators. Hence we have proved 
lem:RLFc Lemma 4.4. Let $E$ be an abelian Lie algebra over $\mathbb{C}$. For any $u \in U(E)$,

$$
\begin{aligned}
& \text { SymbodR }(u)=\overline{d L(u)}, \quad \operatorname{Symb} \circ d L(u)=\overline{d R(u)} \text {. } \\
& \text { thm: } 313
\end{aligned}
$$

43. Proof of Theorem $413(2)$. We are ready to complete the proof of Theorem 4.1 (and Corollary 4.3).

Proof. Take an arbitrary $\varphi \in \operatorname{Hom}_{\mathbb{C}}\left(W^{\vee}, \operatorname{ind}_{\mathfrak{p}}^{\mathfrak{g}}\left(V^{\vee}\right)\right)$, which may be written as a finite sum

$$
\varphi=\sum_{j} u_{j} \otimes \psi_{j} \in U\left(\mathfrak{n}_{-}\right) \otimes \operatorname{Hom}_{\mathbb{C}}(V, W)
$$

by the Poincaré-Bipkhoff-Witt theorem $U(\mathfrak{g}) \simeq U\left(\mathfrak{n}_{-}\right) \otimes U(\mathfrak{p})$. Then it follows from $(2.22)$ and (3.23) that

$$
F_{c} \varphi=\sum_{j} \mathcal{F}_{c}\left(d L\left(u_{j}\right) \delta\right) \otimes \psi_{j} \in \operatorname{Pol}\left(\mathfrak{n}_{+}\right) \otimes \operatorname{Hom}_{\mathbb{C}}(V, W) .
$$

Since $\delta=\mathcal{F}_{c}^{-1}(\mathbf{1})$, we get

$$
F_{c} \varphi=\sum_{j} \widehat{d L\left(u_{j}\right)} \otimes \psi_{j}
$$

On the other hand, by the construction $(2.18)$,

$$
D_{X \rightarrow Y}(\varphi)=\sum_{j} d R\left(u_{j}\right) \otimes \psi_{j}
$$

Now we use the assumption that $\mathfrak{n}_{+}$or equivalently $\mathfrak{n}_{-}$is abelian. Then, in the coordinates $\mathfrak{n}_{-}(\mathbb{R}) \hookrightarrow G / P$ the operator $d R\left(u_{j}\right)$ for $u_{j} \in U\left(\mathfrak{n}_{-}\right)$defines a constant coefficient differential operator on $\mathfrak{n}_{-}$. Thus $D_{X \rightarrow Y}(\varphi)$ can be regarded as an element of $\operatorname{Diff}^{\text {const }}\left(\mathfrak{n}_{-}(\mathbb{R})\right) \otimes \operatorname{Hom}_{\mathbb{C}}(V, W)$.

Applying the symbol map we have

$$
\left.(\operatorname{Symb} \otimes \mathrm{id}) \circ D_{X \rightarrow Y}(\varphi)=\sum_{j} \operatorname{Symb} \circ d R\left(u_{j}\right) \otimes \psi_{j}=\sum_{j} \overline{d L\left(u_{j}\right.}\right) \otimes \psi_{j},
$$

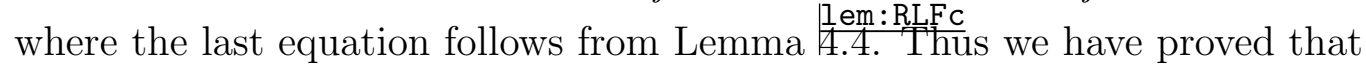

$$
\left(F_{c} \otimes \mathrm{id}\right) \varphi=(\mathrm{Symb} \otimes \mathrm{id}) \circ D_{X \rightarrow Y}(\varphi),
$$

whence the second statement of Theorem 4 thm: 313

4.4. Recipe of the F-method for abelian nilradical $\mathfrak{n}_{+}$. Our goal is to find an explicit form of a differential symmetry breaking operator from $\mathcal{V}_{X}$ to $\mathcal{W}_{Y}$. Equivalently, what we call F-method provides a way to find an explicit element in the space $\operatorname{Hom}_{\mathfrak{g}^{\prime}}\left(\operatorname{ind}_{\mathfrak{p}^{\prime}}^{\mathfrak{g}^{\prime}}\left(W^{\vee}\right), \operatorname{ind}_{\mathfrak{p}}^{\mathfrak{g}}\left(V^{\vee}\right)\right) \simeq \operatorname{Hom}_{\mathfrak{p}^{\prime}}\left(W^{\vee}, \operatorname{ind}_{\mathfrak{p}}^{\mathfrak{g}}\left(V^{\vee}\right)\right)$.

A semisimple element $Z$ in $\mathfrak{g}$ is called hyperbolic if all the eigenvalues of $\operatorname{ad}(Z)$ are real. A hyperbolic element $Z$ defines a parabolic subalgebra $\mathfrak{p}(Z)=\mathfrak{l}(Z)+\mathfrak{n}_{+}(Z)$, where $\mathfrak{l}(Z)$ and $\mathfrak{n}_{+}(Z)$ are the sum of eigenspaces of $\operatorname{ad}(Z)$ with zero and positive eigenvalues, respectively. 
Let $\mathfrak{g}^{\prime}$ be a reductive subalgebra in $\mathfrak{g}$, in the sense that $\mathfrak{g}^{\prime}$ itself is reductive and the adjoint representation of $\mathfrak{g}^{\prime}$ on $\mathfrak{g}$ is completely reducible.

def:3.2 Definition 4.5. A parabolic subalgebra $\mathfrak{p}$ is said to be $\mathfrak{g}^{\prime}$-compatible if there exists a hyperbolic element $Z \in \mathfrak{g}^{\prime}$ such that $\mathfrak{p}=\mathfrak{p}(Z)$.

If $\mathfrak{p}=\mathfrak{l}+\mathfrak{n}_{+}$is $\mathfrak{g}^{\prime}$-compatible, then $\mathfrak{p}^{\prime}:=\mathfrak{p} \cap \mathfrak{g}^{\prime}$ becomes a parabolic subalgebra of $\mathfrak{g}^{\prime}$ with the following Levi decomposition:

$$
\mathfrak{p}^{\prime}=\mathfrak{l}^{\prime}+\mathfrak{n}_{+}^{\prime}:=\left(\mathfrak{l} \cap \mathfrak{g}^{\prime}\right)+\left(\mathfrak{n}_{+} \cap \mathfrak{g}^{\prime}\right),
$$

which satisfies the assumptions of Theorem $\frac{\text { thm }}{4.1} 312$. 213

In this case the space $\operatorname{Diff}_{G^{\prime}}\left(\mathcal{V}_{X}, \mathcal{W}_{Y}\right)$ of differential symmetry breaking operators is always finite-dimensional owing to Corollary 2.10 because:

$$
\operatorname{dim}_{\mathbb{C}} \operatorname{Hom}_{\mathfrak{g}^{\prime}}\left(\operatorname{ind}_{\mathfrak{p}^{\prime}}^{\mathfrak{g}^{\prime}}\left(W^{\vee}\right), \operatorname{ind}_{\mathfrak{p}}^{\mathfrak{g}}\left(V^{\vee}\right)\right)<\infty
$$

for any finite-dimensional representations $V$ and $W$ of $\mathfrak{p}$ and $\mathfrak{p}^{\prime}$, respectively 䏝14, Proposition 2.8].

Our assumption here is that $\mathfrak{p}=\mathfrak{l}+\mathfrak{n}_{+}$is a $\mathfrak{g}^{\prime}$-compatible parabolic subalgebra of $\mathfrak{f}_{\text {fod }}$ with abelian nilradical $\mathfrak{n}_{+}$. Based on the following diagram (see Corollary 4.3),

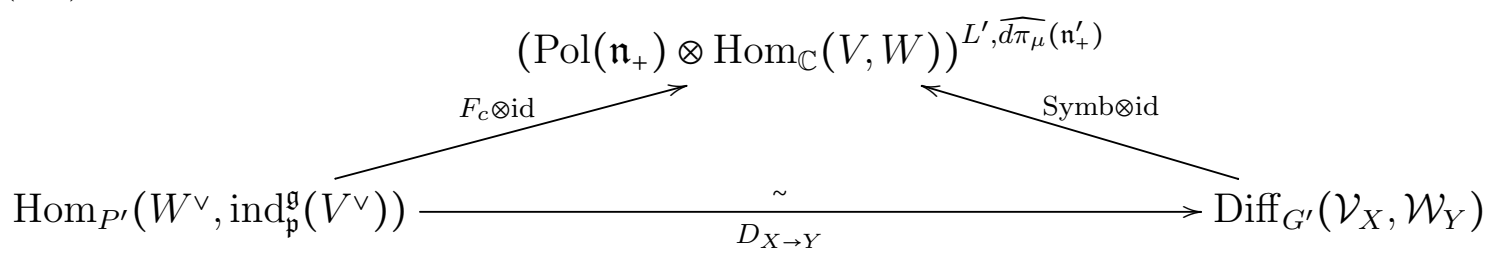

we develop a method as follows:

Step 0. Fix a finite-dimensional representation $(\lambda, V)$ of the parabolic subgroup $P$. It defines a $G$-equivariant vector bundle $\mathcal{V}_{X}=G \times_{P} V$ over $X=G / P$.

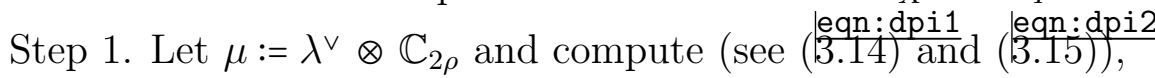

$$
\begin{aligned}
& d \pi_{\mu}: \mathfrak{g} \rightarrow \mathcal{D}\left(\mathfrak{n}_{-}\right) \otimes \operatorname{End}\left(V^{\vee}\right), \\
& \widehat{d \pi_{\mu}}: \mathfrak{g} \rightarrow \mathcal{D}\left(\mathfrak{n}_{+}\right) \otimes \operatorname{End}\left(V^{\vee}\right) .
\end{aligned}
$$

According to $\left(\frac{\operatorname{egn} \cdot 3.13), d \pi_{\mu}}{3.13}\right.$ only depends on the infinitesimal representation $\lambda$ of the parabolic subalgebra $\mathfrak{p}$.

Step 2. Find a finite-dimensional representation $(\nu, W)$ of the Lie group $P^{\prime}$ such that

$$
\operatorname{Hom}_{P^{\prime}}\left(W^{\vee}, \operatorname{ind}_{\mathfrak{p}}^{\mathfrak{g}}\left(V^{\vee}\right)\right) \neq\{0\} .
$$

It defines a $G^{\prime}$-equivariant vector bundle $\mathcal{W}_{Y}=G^{\prime} \times_{P^{\prime}} W$ over $Y=G^{\prime} / P^{\prime}$ such that $\operatorname{Diff}_{G^{\prime}}\left(\mathcal{V}_{X}, \mathcal{W}_{Y}\right)$ is non-trivial. 


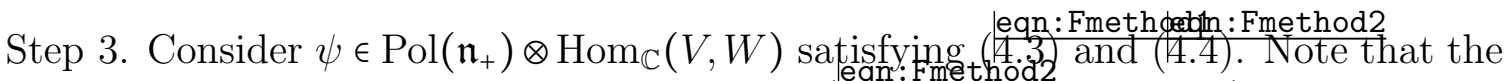
s.sstem of partial differential equations $(4.4)$ is of second order (see Proposition 3.10).

Step 4. Take a slice $S$ for generic $L_{\mathbb{C}^{-}}^{\prime}$ orbits on $\mathfrak{n}_{+}$. Use invariant theory for $\frac{\text { lean: Fmethod1 }}{4: 3 \text { and }}$ consider the system of differential equations on $S$ induced from a 4.4 . Hind polynomials $\psi \in \operatorname{Pol}\left(\mathfrak{n}_{+}\right) \otimes \operatorname{Hom}(V, W)$ satisfying (4.3) and $(4.4)$ by solving those equations on $S$.

Step 5. Let $\psi$ be a polynomial solution to 4.3 and and 4.4 obtained in Step 4 . In

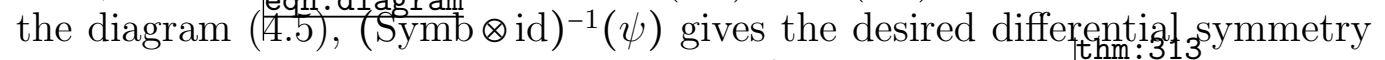
breaking operator in the coordinates $\mathfrak{n}_{-}$of $X$ by Theorem 4.1 . In the same diagram, $\left(F_{c} \otimes \mathrm{id}\right)^{-1}(\psi)$ gives an explicit element in $\operatorname{Hom}_{\mathfrak{p}^{\prime}}\left(W^{\vee}, \operatorname{ind}_{\mathfrak{p}}^{\mathfrak{g}}\left(V^{\vee}\right)\right)$ $\left(\simeq \operatorname{Hom}_{\mathfrak{g}^{\prime}}\left(\operatorname{ind}_{\mathfrak{p}^{\prime}}^{\mathfrak{g}^{\prime}}\left(W^{\vee}\right), \operatorname{ind}_{\mathfrak{p}}^{\mathfrak{g}}\left(V^{\vee}\right)\right)\right)$, which is sometimes referred to as a singular vector.

This method gives all non-trivial differential symmetry breaking operators for given data $\left(Y \hookrightarrow X, \mathcal{V}_{X}\right)$ by providing $G^{\prime}$-equivariant vector bundles $\mathcal{W}_{Y_{j}}$ and explicit elements in $\operatorname{Diff}_{G^{\prime}}\left(\mathcal{V}_{X}, \mathcal{W}_{Y}\right)$. In fact, Step 2 based on Theorem 2.9 gives a necessary and sufficient condition for a $P^{\prime}$-module $W$ to ${ }^{\prime}$ ensure that $\operatorname{Diff}_{G^{\prime}}\left(\mathcal{V}_{X}, \mathcal{W}_{Y}\right)$ is non-zero. Steps 1, 3 and 5 based on Theorem 4.1 show that any differential symmetry breaking operator is of the form $\left(F_{c} \otimes \mathrm{id}\right)^{-1}(\psi)$ where $\psi$ is a polynomial solution to (4.3) and 4.4 .

Actual applications of the F-method include the following cases:

1. Holomorphic discrete series representations.

2. Principal series representations of real reductive groups (Corollary 2.13 . holoreal

The latter is related foquestions in conformal geometry (more generally parabolic geometry), see [J09, KØSS13]. The former case includes the classical Rankin-Cohen bidifferential Operators as a prototype, and it is the main object of the second part of this work [KPI5-2]. The connection between thesentwo is discussed in KKP15. We note that the normalization and formulation in [KסSS13] is different from this paper, see [K13].

Here we give some comments on the actual applications of the F-method when $X$ and $Y$ are Hermitian symmetric spaces. In Theorem 5.3 we prove that all continuous symmetry breaking operators in this case are given by holomorphic differential operators that extend to the complex flag varieties, so that the F-method for a parabolic subalgebra with abelian nilradical applies.

Furthermore, if $\left(G, G^{\prime}\right)$ is a reductive symmetric pair, we know a priori that $\operatorname{Diff}_{G^{\prime}}\left(\mathcal{V}_{X}, \mathcal{W}_{Y}\right)$ is one-dimensional for line bundles $\mathcal{V}_{X}$ with generic parameter $[$ K 14 , Theorem 2.7]. Thus, it is natural to look for explicit formulpe for such canonical operators. In Step 2 we can use explicit branching laws (see [KPI5-2, Fact ???]) to find 
all $W$ such that $\operatorname{Hom}_{\mathfrak{p}^{\prime}}\left(W^{\vee}, \operatorname{ind}_{\mathfrak{p}}^{\mathfrak{g}}\left(V^{\vee}\right)\right)$ is non-zero. Conversely, the differential equations in Step 3 are useful in certain cases to get a finer structure of branching laws, e.g., to find the Jordan-Hölder series of the restriction for exceptional parameters $\lambda$ (see [1ØSS13]).

The Rankin-Cohen operators as well as Juhl's conformally covariant differential operators are recovered by the F-method as a special case where generic $L_{\mathbb{C}}^{\prime}$-orbits on $\mathfrak{n}_{+}$are of codimension one. The induced system of 4.4 reduces to ordinary differential equations on the one dimensional complex manifold $S$. In the second part of this work [KPI5-2] we shall treat all the six geometries with a one-dimensional slice $S$.

subsec: 36

4.5. F-method - supplement for vector valued cases. In order to deal with the general case where the target $\mathcal{W}_{Y}$ is no longer a line bundle but a vector bundle, i.e., where $W$ is an arbitrary finite-dimensional irreducible $\mathfrak{l}^{\prime}$-module, we may find the condition s.3 somewhat complicated in practice, even though it is a system of differential equations of first order. In this section we give two useful lemmas to simplify Step 3 in the recipe by reducing t4.3 to a simpler algebraic question on polynomial rings, so that we can focus on the crucial part consisting of a spstem of differential equations of second order 4.4 . The results here will be used in IKP15-2,

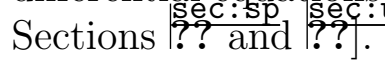

We fix a Borel subalgebra $\mathfrak{b}\left(\mathfrak{l}^{\prime}\right)$ of $\mathfrak{l}^{\prime}$. Let $\chi: \mathfrak{b}\left(\mathfrak{l}^{\prime}\right) \rightarrow \mathbb{C}$ be a character. For an $\mathfrak{l}^{\prime}$-module $U$, we set

$$
U_{\chi}:=\left\{u \in U: Z u=\chi(Z) u \text { for any } Z \in \mathfrak{b}\left(\mathfrak{l}^{\prime}\right)\right\} .
$$

Suppose that $W$ is an irreducible representation of $\mathfrak{l}^{\prime}$ with lowest weight $-\chi$. Then the contragredient representation $W^{\vee}$ has a highest weight $\chi$. We fix a non-zero highest weight vector $w^{\vee} \in\left(W^{\vee}\right)_{\chi}$. Then the contraction map

$$
U \otimes W \rightarrow U, \quad \psi \mapsto\left\langle\psi, w^{\vee}\right\rangle,
$$

induces a bijection between the following two subspaces:

eqn : 111

$$
(U \otimes W)^{\mathfrak{l}^{\prime}} \stackrel{\sim}{\longrightarrow} U_{\chi},
$$

if $U$ is completely reducible as an $\mathfrak{l}^{\prime}$-module. By using the isomorphism $\frac{\text { ean: } 111}{\left(\frac{1.6}{6}\right), \text { we }}$ reformulate Step 3 of the recipe for the F-method as follows:

lem:412 Lemma 4.6. Suppose we are in the setting of Section 4.4. Assume that $W$ is an irreducible representation of the parabolic subalgebra $\mathfrak{p}^{\prime}$. Let $-\chi$ be the lowest weight of $W$ as an $\mathfrak{l}^{\prime}$-module. Then we have a natural injective homomorphism

$$
\operatorname{Diff}_{G^{\prime}}\left(\mathcal{V}_{X}, \mathcal{W}_{Y}\right) \hookrightarrow\left\{Q \in\left(\operatorname{Pol}\left(\mathfrak{n}_{+}\right) \otimes V^{\vee}\right)_{\chi}: \widehat{d \pi_{\mu}}(C) Q=0 \quad \text { for all } C \in \mathfrak{n}_{+}^{\prime}\right\},
$$

which is bijective if $L^{\prime}$ is connected. 


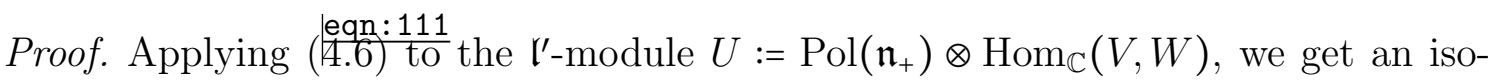
morphism:

eqn :PVWchi

$$
\left(\operatorname{Pol}\left(\mathfrak{n}_{+}\right) \otimes \operatorname{Hom}(V, W)\right)^{\mathfrak{l}^{\prime}} \stackrel{\sim}{\longrightarrow}\left(\operatorname{Pol}\left(\mathfrak{n}_{+}\right) \otimes V^{\vee}\right)_{\chi}
$$

Since $W$ is an irreducible $\mathfrak{p}^{\prime}$-module, the Lie subalgebra $\mathfrak{n}^{\prime}$ acts trivially on $W$ and $\mathfrak{l}^{\prime}$ acts irreducibly. In particular, the condition (4.4) amounts to

$$
\left(\widehat{d \pi_{\mu}}(C) \otimes \mathrm{id}_{W}\right) \psi=0 \quad \text { for all } C \in \mathfrak{n}_{+}^{\prime} .
$$

Therefore, the isomorphism $\frac{\text { eqn }}{4.7}$ : PVWchi induces a bijection

$$
\begin{aligned}
& \left\{\psi \in\left(\operatorname{Pol}\left(\mathfrak{n}_{+}\right) \otimes \operatorname{Hom}(V, W)\right)^{l^{\prime}}: \psi \text { satisfies } \frac{\text { eqn }}{4.4}\right\} \text { Fmethod2 } \\
\stackrel{\sim}{\rightarrow} & \left\{Q \in\left(\operatorname{Pol}\left(\mathfrak{n}_{+}\right) \otimes V^{\vee}\right)_{\chi}: \widehat{d \pi_{\mu}}(C) Q=0 \text { for all } C \in \mathfrak{n}_{+}^{\prime}\right\} .
\end{aligned}
$$

Now Lemma follows from Theorem 4 thm: 313

Since any non-zero vector in $W^{\vee}$ is cyclic, the next lemma explains how to recover $D_{X \rightarrow Y}(\varphi)$ from $Q$ given in Lemma 4.6 .

We assume, for simplicity, that the $\mathfrak{l}$-module $(\lambda, V)$ lifts to $L_{\mathbb{C}}$, the $\mathfrak{I}^{\prime}$-module $(\nu, W)$ lifts to $L_{\mathbb{C}}^{\prime}$, and use the same letters to denote their liftings.

$$
\left\langle D_{X \rightarrow Y}(\varphi), \nu^{\vee}(\ell) w^{\vee}\right\rangle=\left(\operatorname{Ad}(\ell) \otimes \lambda^{\vee}(\ell)\right)\left\langle D_{X \rightarrow Y}(\varphi), w^{\vee}\right\rangle .
$$

Proof. We write $\varphi=\sum_{j} u_{j} \otimes \psi_{j} \in U\left(\mathfrak{n}_{-}\right) \otimes \operatorname{Hom}_{\mathbb{C}}(V, W)$. Since $\varphi$ is $\mathfrak{p}^{\prime}$-invariant, we have the identity:

$$
\sum_{j} u_{j} \otimes \psi_{j}=\sum_{j} \operatorname{Ad}(\ell) u_{j} \otimes \nu(\ell) \circ \psi_{j} \circ \lambda\left(\ell^{-1}\right) \quad \text { for } \mathfrak{l} \in L_{\mathbb{C}}^{\prime} .
$$

In turn, we have

$$
\begin{aligned}
\left\langle D_{X \rightarrow Y}(\varphi), \nu^{\vee}(\ell) w^{\vee}\right\rangle & =\sum_{j} d R\left(\operatorname{Ad}(\ell) u_{j}\right) \otimes\left\langle\psi_{j}, w^{\vee}\right\rangle \circ \lambda\left(\ell^{-1}\right) \\
& =\left(\left(\operatorname{Ad}(\ell) \otimes \lambda^{\vee}(\ell)\right)\left\langle D_{X \rightarrow Y}(\varphi), w^{\vee}\right\rangle .\right.
\end{aligned}
$$

Thus, we have proved Lemma.

We notice that the right-hand side of $\frac{\text { ean: }}{4.8 c a l e a}$ can be computed by using the identity in $\operatorname{Diff}^{\text {const }}\left(\mathfrak{n}_{-}\right) \otimes V^{\vee}$ :

$$
\left\langle D_{X \rightarrow Y}(\varphi), w^{\vee}\right\rangle=\left(\operatorname{Symb}^{-1} \otimes \operatorname{id}_{V^{\vee}}\right)(Q),
$$

once we know the polynomial $Q=\left\langle\psi, w^{\vee}\right\rangle$ with $\psi=\left(F_{c} \otimes\right.$ id) $(\varphi)$ (see Theorem Sec:sp Sec:unt In [KPI5-2, Sections ??? and ??], we find explicit formulæ for vector-bundle valued equivariant differential operators by solving equations for the polynomials $Q$. 


\section{LOCALNESS AND EXTENSION THEOREM FOR SYMMETRY BREAKING} OPERATORS

Let $G \supset G^{\prime}$ be a pair of real reductive Lie groups. In general, continuous symmetry breaking operators between two principal series representations of $G$ and $G^{\prime}$ are not always given by differential operators. Actually, generic ones are supposed to be given by integral transforms and their meromorphic continuation, as one can see from a classification result [KS14]. In this section, however, we formulate and prove a quite remarkable phenomenon (localness theorem) that any continuous $G^{\prime}$ intertwining operator between two representation spaces consisting of holomorphic sections oyer Hermitian symmetric spaces is given by differential operators, see Theorem 5.3 . In particular, the covariant holomporphic differential operators which we shall obtain explicitly in the second part [KP15-2] of this work exhaust all continuous symmetry breaking operators.

5.1. Formulation of the localness theorem. Let $G$ be a connected reductive Lie group, $\theta$ a Cartan involution, and $G / K$ the associated Riemannian symmetric space. We write $\mathfrak{c}(\mathfrak{k})$ for the center of the complexified Lie algebra $\mathfrak{k}:=\operatorname{Lie}(K) \otimes_{\mathbb{R}} \mathbb{C} \equiv$ $\mathfrak{k}(\mathbb{R}) \otimes_{\mathbb{R}} \mathbb{C}$. In order to formulate a localness theorem, we suppose that $G / K$ is a Hermitian symmetric space. This means that there exists a characteristic element $Z \in \mathfrak{c}(\mathfrak{k})$ such that the eigenvalues of $\operatorname{ad}(Z) \in \operatorname{End}(\mathfrak{g})$ is 0 or \pm 1 and that we have an eigenspace decomposition

$$
\mathfrak{g}=\mathfrak{k}+\mathfrak{n}_{+}+\mathfrak{n}_{-}
$$

of $\operatorname{ad}(Z)$ with eigenvalues 0,1 , and -1 , respectively. We note that $\mathfrak{c}(\mathfrak{k})$ is onedimensional if $G$ is simple. With the notation of the previous sections, the complex Lie algebra $\mathfrak{k}$ plays the role of the Levi subalgebra $\mathfrak{l}$.

Let $G_{\mathbb{C}}$ be a complex reductive Lie group with Lie algebra $\mathfrak{g}$, and $P_{\mathbb{C}}$ the maximal parabolic subgroup with Lie algebra $\mathfrak{p}:=\mathfrak{k}+\mathfrak{n}_{+}$, with abelian nilradical $\mathfrak{n}_{+}$. The complex structure of the homogeneous $G / K$ is induced from the open embedding

$$
G / K \subset G_{\mathbb{C}} / K_{\mathbb{C}} \exp \mathfrak{n}_{+}=G_{\mathbb{C}} / P_{\mathbb{C}}
$$

Let $G^{\prime}$ be a connected reductive subgroup of $G$. Without loss of generality we may and do assume that $G^{\prime}$ is $\theta$-stable. We set $K^{\prime}:=K \cap G^{\prime}$. Our crucial assumption throughout this section is

$$
Z \in \mathfrak{k}^{\prime}
$$

lem:subherm

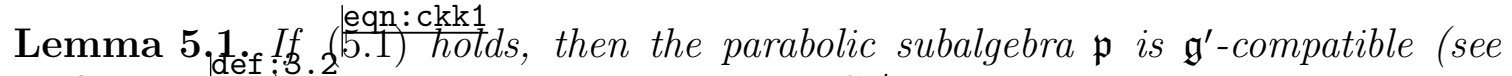
Definition 4.5j, and the homogeneous space $G^{\prime} / K^{\prime}$ is a Hermitian sub-symmetric space of $G / K$ such that the embedding $G^{\prime} / K^{\prime} \rightarrow G / K$ is holomorphic.

Proof. Let $G_{\mathbb{C}}^{\prime}$ be the connected complex subgroup of $G_{\mathbb{C}}$ with Lie algebra $\mathfrak{g}^{\prime}:=$ $\operatorname{Lie}\left(G^{\prime}\right) \otimes_{\mathbb{R}} \mathbb{C}$. Then $\mathfrak{p}^{\prime}:=\mathfrak{k}^{\prime}+\mathfrak{n}_{+}^{\prime} \equiv\left(\mathfrak{k} \cap \mathfrak{g}^{\prime}\right)+\left(\mathfrak{n}_{+} \cap \mathfrak{g}^{\prime}\right)$ is the sum of the eigenspaces 
of $\operatorname{ad}(Z)$ in $\mathfrak{g}^{\prime}$ with 0 and +1 eigenvalues, respectively, and therefore is a parabolic subalgebra of $\mathfrak{g}^{\prime}$. We set $P_{\mathbb{C}}^{\prime}:=P_{\mathbb{C}} \cap G^{\prime}$. Then, the Riemannian symmetric space $G^{\prime} / K^{\prime}$ becomes a Hermitian symmetric space, for which the complex structure is induced from the open embedding in the complex flag variety $Y_{\mathbb{C}}:=G_{\mathbb{C}}^{\prime} / P_{\mathbb{C}}^{\prime}$ :

$$
\begin{gathered}
G^{\prime} / K^{\prime} \rightarrow G / K \\
Y_{\mathbb{C}}=G_{\mathbb{C}}^{\prime \text { open }} \bigcap_{\mathbb{C}}^{\prime} \rightarrow G_{\mathbb{C}} / P_{\mathbb{C}}=X_{\mathbb{C}} .
\end{gathered}
$$

Since $Y_{\mathbb{C}}$ is a complex submanifold of $X_{\mathbb{C}}=G_{\mathbb{C}} / P_{\mathbb{C}}$, the embedding $G^{\prime} / K^{\prime} \hookrightarrow G / K$ is holomorphic.

Notice that in the setting of Lemma the complexified Lie algebra of $K^{\prime}$ is a Levi subalgebra of the parabolic subalgebra $\mathfrak{p}^{\prime}$.

ex:ckk Example 5.2. (1) Let $G^{\prime}$ be a connected simple Lie group such that the associated Riemannian symmetric space $G^{\prime} / K^{\prime}$ is a Hermitian symmetric space. We take a characteristic element $Z^{\prime} \in \mathfrak{c}\left(\mathfrak{k}^{\prime}\right)$. Let $G:=G^{\prime} \times G^{\prime}$, and we realize $G^{\prime}$ as the diagonal subgroup. $A\left(G^{\prime}\right):=\left\{(g, g): g \in G^{\prime}\right\}$ of $G$. Then $Z:=\left(Z^{\prime}, Z^{\prime}\right) \in \mathfrak{c}(\mathfrak{k})$ satisfies (5.1), yielding a holomorphic embedding $\Delta$ : $G^{\prime} / K^{\prime} \hookrightarrow G / K=G^{\prime} / K^{\prime} \times G^{\prime} / K^{\prime}$.

(2) Let $G$ be a connected simple Lie group such that the associated symmetric space $G / K$ is a Hermitian symmetric space with $Z$ a characteristic element in $\mathfrak{c}(\mathfrak{k})$. Suppose $\tau$ is an automorphism of $G$ of finite order such that $\tau(Z)=Z$. Let $G^{\prime}$ be the identity component of the subqroup $G^{\tau}:=\{g \in G: \tau(g)=g\}$, and $K^{\prime}:=G^{\prime} \cap K$. Then the assumption (T) is satisfied, and $G^{\prime} / K^{\prime}$ is a Hermitian sub-symmetric space of $G / K$. We shall focyson the case where $\left(G, G^{\tau}\right)$ is a symmetric pair, namely, $\tau$ is of order two in [KPI5-2] for detailed analysis.

Consider a finite-dimensional representation of $K$ on a complex vector space $V$. We extend it to a holomorphic representation of $P_{\mathbb{C}}$ by letting the unipotent subgroup $\exp \left(\mathfrak{n}_{+}\right)$act trivially, and form a holomorphic vector bundle $\mathcal{V}_{X_{\mathbb{C}}}=G_{\mathbb{C}} \times_{P_{\mathbb{C}}} V$ over $X_{\mathbb{C}}=$ $G_{\mathbb{C}} / P_{\mathbb{C}}$. The restriction to the open set $G / K$ defines a $G$-equivariant holomorphic vector bundle $\mathcal{V}:=G \times_{K} V$. We then have a natural representation of $G$ on the vector space $\mathcal{O}(G / K, \mathcal{V})$ of global holomorphic sections endowed with the Fréchet topology of uniform convergence on compact sets.

Likewise, given a finite-dimensional representation $W$ of $K^{\prime}$, we form the $G^{\prime}$ equivariant holomorphic vector bundle $\mathcal{W}=G^{\prime} \times_{K^{\prime}} W$ and consider the representation of $G^{\prime}$ on $\mathcal{O}\left(G^{\prime} / K^{\prime}, \mathcal{W}\right)$. By definition, it is clear that

eqn : dcont

Theorem 5.3 below shows that the two spaces do coincide.

$$
\operatorname{Diff}_{G^{\prime}}^{\text {hol }}\left(\mathcal{V}_{X}, \mathcal{W}_{Y}\right) \subset \operatorname{Hom}_{G^{\prime}}\left(\mathcal{O}(G / K, \mathcal{V}), \mathcal{O}\left(G^{\prime} / K^{\prime}, \mathcal{W}\right)\right)
$$


thm:C Theorem 5.3. Let $G^{\prime}$ be a reductive subgroup of $G$ satisfying (5.1). Let $V$ and $W$ be any finite-dimensional representations of $K$ and $K^{\prime}$, respectively. Then,

(1) (localness theorem) any continuous $G^{\prime}$-homomorphism from $\mathcal{O}(G / K, \mathcal{V})$ to $\mathcal{O}\left(G^{\prime} / K^{\prime}, \mathcal{W}\right)$ is given by a holomorphic differential operator, in the sense of Definition [2.1. with respect to a holomorphic map between the Hermitian symmetric spaces $G^{\prime} / K^{\prime} \rightarrow G / K$, that is,

$$
\operatorname{Diff}_{G^{\prime}}^{\text {hol }}\left(\mathcal{V}_{X}, \mathcal{W}_{Y}\right)=\operatorname{Hom}_{G^{\prime}}\left(\mathcal{O}(G / K, \mathcal{V}), \mathcal{O}\left(G^{\prime} / K^{\prime}, \mathcal{W}\right)\right)
$$

(2) (extension theorem) any such a differential operator (or equivalently, any continuous $G^{\prime}$-homomorphism) extends to a $G_{\mathbb{C}}^{\prime}$-equivariant holomorphic differential operator with respect to a holomorphic map between the flag varieties $Y_{\mathbb{C}}=G_{\mathbb{C}}^{\prime} / P_{\mathbb{C}}^{\prime} \hookrightarrow X_{\mathbb{C}}=G_{\mathbb{C}} / P_{\mathbb{C}}$, namely, the injection

eqn:inj

$$
\operatorname{Difff}_{G_{\mathbb{C}}^{\prime}}^{\text {hol }}\left(\mathcal{V}_{X_{\mathbb{C}}}, \mathcal{W}_{Y_{\mathbb{C}}}\right) \hookrightarrow \operatorname{Difff}_{G^{\prime}}^{\text {hol }}\left(\mathcal{V}_{X}, \mathcal{W}_{Y}\right)
$$

is bijective.

Remark 5.4. More generally, we may ask whether an analogous statement to Theorem 5.3 (1) holds or not if we replace $\mathcal{O}(G / K, \mathcal{V})$ and $\mathcal{O}\left(G^{\prime} / K^{\prime}, \mathcal{W}\right)$ by some other topological vector spaces having the same underlying $(\mathfrak{g}, K)$-module and $\left(\mathfrak{g}^{\prime}, K^{\prime}\right)$-module, respectively (e.g. the Casselman-Wallach globalization, Hilbert space globalization, etc.). This question was raised by D. Vogan in May 2014. It turns out that this generalization is also true, as we shall show in the proof of Theorem 5.3, that the natural injection

eqn:gKsurj

$$
\operatorname{Difff}_{G_{\mathbb{C}}^{\prime}}^{\text {hol }}\left(\mathcal{V}_{X_{\mathbb{C}}}, \mathcal{W}_{Y_{\mathbb{C}}}\right) \hookrightarrow \operatorname{Hom}_{\left(\mathfrak{g}^{\prime}, K^{\prime}\right)}\left(\mathcal{O}(G / K, \mathcal{V})_{K \text {-finite }}, \mathcal{O}\left(G^{\prime} / K^{\prime}, \mathcal{W}\right)_{K^{\prime} \text {-finite }}\right)
$$

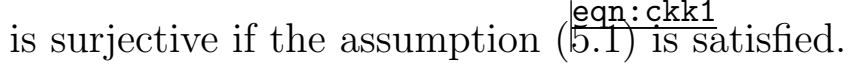

Remark 5.5. An analogous statement for real parabolic subgroups is not true. For instance, for the pair $\left(G, G^{\prime}\right)=(O(n+1,1), O(n, 1))$ there always exists a non-zero continuous $G^{\prime}$-equivariant map from the spherical principal series representations $C^{\infty}\left(G / P, \mathcal{L}_{\lambda}\right)$ of $G$ to the one $C^{\infty}\left(G^{\prime} / P^{\prime}, \mathcal{L}_{\nu}\right)$ of $G^{\prime}$ for any $(\lambda, \nu) \in \mathbb{C}^{2}$, however, non-zero $G^{\prime}$-equivariant differential operators exist if and only if $\nu-\lambda \in 2 \mathbb{N}$ [KS14.

Remark 5.6. Suppose that $V$ is a generic character of $K$ and $\left(G, G^{\prime}\right)$ is a symmetric

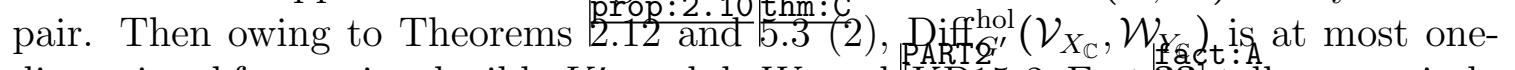
dimensional for any irreducible $K^{\prime}$-module $W$, and IIKP15-2, Fact ??? tells us precisely when it is non-zero.

PART2

In $[$ KPI5-2] we describe explicit formulæ of such differential operators by using the F-method (Theorem f.1) for the six complex geometries arising from symmetric pairs of split rank one. 
5.2. Proof of the localness theorem. Theorem discretely decomposable restrictions (see [K94, K98a, K98b]). The proof is based on a careful analysis of the following three objects:

$$
(\mathfrak{g}, K) \text {-modules, }\left(\mathfrak{g}, K^{\prime}\right) \text {-modules, and }\left(\mathfrak{g}^{\prime}, K^{\prime}\right) \text {-modules. }
$$

We say that a $K^{\prime}$-module $Z$ is $K^{\prime}$-admissible if the multiplicity

$$
[M: F]:=\operatorname{dim} \operatorname{Hom}_{K^{\prime}}\left(F,\left.M\right|_{K^{\prime}}\right)
$$

is finite for any $F \in \widehat{K^{\prime}}$. Then, $K^{\prime}$-admissibility is preserved by taking the tensor product with finite-dimensional representations.

We write $\mathcal{O}(G / K, \mathcal{V})_{K \text {-finite }}$ for the space of $K$-finite vectors of $\mathcal{O}(G / K, \mathcal{V})$, which becomes naturally a $(\mathfrak{g}, K)$-module.

lem:431 Lemma 5.7. The $(\mathfrak{g}, K)$-module $\mathcal{O}(G / K, \mathcal{V})_{K \text {-finite }}$ is $K^{\prime}$-admissible if $Z \in \mathfrak{g}^{\prime}$.

Proof. As a $K$-module, we have the following isomorphism

$$
\begin{aligned}
\mathcal{O}(G / K, \mathcal{V})_{K \text {-finite }} & \simeq S\left(\mathfrak{n}_{+}\right) \otimes V \\
& \simeq\left(\bigoplus_{a \geq 0} S^{a}\left(\mathfrak{n}_{+}\right)\right) \otimes V
\end{aligned}
$$

where $S^{a}\left(\mathfrak{n}_{+}\right)$denotes the space of symmetric tensors of homogeneous degree $a$. Since $\exp (\mathbb{R} \sqrt{-1} Z)$ acts on $S^{a}\left(\mathfrak{n}_{+}\right)$as the scalar $e^{\sqrt{-1} a t}(t \in \mathbb{R})$, the whole $S\left(\mathfrak{n}_{+}\right)$ is admissible as a module of the one-dimensional subgroup $\exp (\mathbb{R} \sqrt{-1} Z)$ and so is

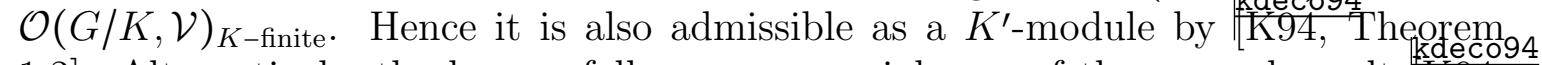
1.2]. Alternatively, the lemma follows as a special case of the general result [K94, Theorem 2.7] or [K98a, Theorem 4.1].

Given a $\left(\mathfrak{g}, K^{\prime}\right)$-module $M$, we consider the contragredient representation on the dual space $M^{\vee}:=\operatorname{Hom}_{\mathbb{C}}(M, \mathbb{C})$. Collecting $K^{\prime}$-finite vectors in $M^{\vee}$, we get a $\left(\mathfrak{g}, K^{\prime}\right)$ module $\left(M^{\vee}\right)_{K^{\prime} \text {-finite }}$.

lem:432 Lemma 5.8. Let $M$ be a $K^{\prime}$-admissible $\left(\mathfrak{g}, K^{\prime}\right)$-module. Then,

(1) $M$ is discretely decomposable as a $\left(\mathfrak{g}^{\prime}, K^{\prime}\right)$-module.

(2) The $\left(\mathfrak{g}, K^{\prime}\right)$-module $\left(M^{\vee}\right)_{K^{\prime} \text {-finite }}$ is $K^{\prime}$-admissible and one has the following $K^{\prime}$-isomorphism

$$
\left(M^{\vee}\right)_{K^{\prime} \text {-finite }} \simeq \bigoplus_{F \in \bar{K}^{\prime}}[M: F] F^{\vee}
$$

For the proof we refer to $\left[\frac{\| d e r n 98}{[\mathrm{~K} 98 \mathrm{~b}}\right.$, Proposition 1.6].

lem:433 Lemma 5.9. Let $M$ be a $K^{\prime}$-admissible $(\mathfrak{g}, K)$-module. Then,

$$
\left(M^{\vee}\right)_{K \text {-finite }}=\left(M^{\vee}\right)_{K^{\prime} \text {-finite }} .
$$


Proof. There is an obvious inclusion $\left(M^{\vee}\right)_{K \text {-finite }} \subset\left(M^{\vee}\right)_{K^{\prime} \text {-finite }}$. We shall prove that the multiplicities in $\left(M^{\vee}\right)_{K \text {-finite }}$ and $\left(M^{\vee}\right)_{K^{\prime} \text {-finite }}$ are both finite and are the same. Indeed, $M$ being $K^{\prime}$-admissible, one has

$$
[M: F]=\bigoplus_{E \in \widehat{K}}[M: E][E: F]<\infty .
$$

Conversely, $\left(M^{\vee}\right)_{K^{\prime} \text {-finite }} \simeq \bigoplus_{F \in \widehat{K^{\prime}}}[M: F] F^{\vee}$ and thus,

$$
\left(M^{\vee}\right)_{K \text {-finite }} \simeq \bigoplus_{E \in \widehat{K}}[M: E] E^{\vee} \simeq \bigoplus_{F \in \widehat{K}^{\prime}}\left(\bigoplus_{E \in \widehat{K}}[M: E][E: F]\right) F^{\vee},
$$

which concludes the proof.

The next lemma is known to experts, but for the sake of completeness, we give a proof.

lem:434 Lemma 5.10. There is a natural $(\mathfrak{g}, K)$-isomorphism:

$$
\left(\left(\mathcal{O}(G / K, \mathcal{V})_{K \text {-finite }}\right)^{\vee}\right)_{K \text {-finite }} \simeq \operatorname{ind}_{\mathfrak{p}}^{\mathfrak{g}}\left(V^{\vee}\right)
$$

Proof. As in Lemma 2.15 , there is a natural non-degenerate $\mathfrak{g}$-invariant bilinear form

$$
\mathcal{O}(G / K, \mathcal{V})_{K \text {-finite }} \times \operatorname{ind}_{\mathfrak{p}}^{\mathfrak{g}}\left(V^{\vee}\right) \rightarrow \mathbb{C} .
$$

Hence, we have an injective $(\mathfrak{g}, K)$-homomorphism $\operatorname{ind}_{\mathfrak{p}}^{\mathfrak{g}}\left(V^{\vee}\right) \subset\left(\mathcal{O}(G / K, \mathcal{V})_{K \text {-finite }}\right)^{\vee}$. Taking $K$-finite vectors we get the following commutative diagram of $K$-modules isomorphisms:

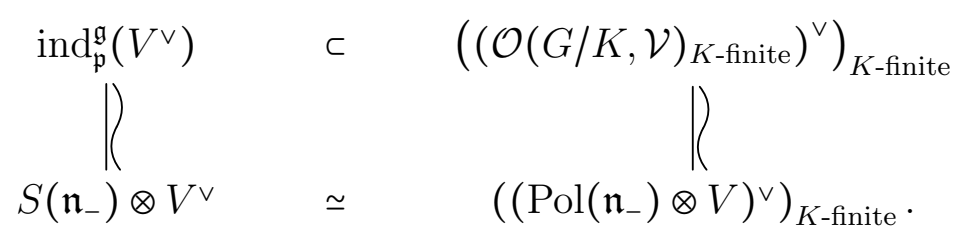

Hence the first row is also bijective.

Combining Lemmas $5.7,5.9$ and 5.10 we have shown the following key result:

prop:Kprimeind Proposition 5.11. There is a natural $(\mathfrak{g}, K)$-isomorphism:

$$
\left(\left(\mathcal{O}(G / K, \mathcal{V})_{K \text {-finite }}\right)^{\vee}\right)_{K^{\prime} \text {-finite }} \simeq \operatorname{ind}_{\mathfrak{p}}^{\mathfrak{g}}\left(V^{\vee}\right)
$$

Proof of Theorem $\stackrel{t h m}{5.3}$. C Let $T: \mathcal{O}(G / K, \mathcal{V}) \rightarrow \mathcal{O}\left(G^{\prime} / K^{\prime}, \mathcal{W}\right)$ be a continuous $G^{\prime}$-intertwining operator. It induces a $\left(\mathfrak{g}^{\prime}, K^{\prime}\right)$-homomorphism

eqn : TK

$$
T_{K}: \mathcal{O}(G / K, \mathcal{V})_{K \text {-finite }} \rightarrow \mathcal{O}\left(G^{\prime} / K^{\prime}, \mathcal{W}\right)_{K^{\prime} \text {-finite }}
$$

We shall prove that any such $\left(\mathfrak{g}^{\prime}, K^{\prime}\right)$-homomorphism $T_{K}$ comes from a $G_{\mathbb{C}}^{\prime}$-equivariant differential operator on the flag variety. 
To see this, we take the dual map $\frac{\text { ag }}{5.5), \text { TK }}$, and apply Lemma 5.10 and Proposition 5.11. Then there is a $\left(\mathfrak{g}^{\prime}, K^{\prime}\right)$-homomorphism $\psi: \operatorname{ind}_{\mathfrak{p}^{\prime}}^{\mathfrak{g}^{\prime}}\left(W^{\vee}\right) \rightarrow \operatorname{ind}_{\mathfrak{p}}^{\mathfrak{g}}\left(V^{\vee}\right)$ such that the following diagram commutes:

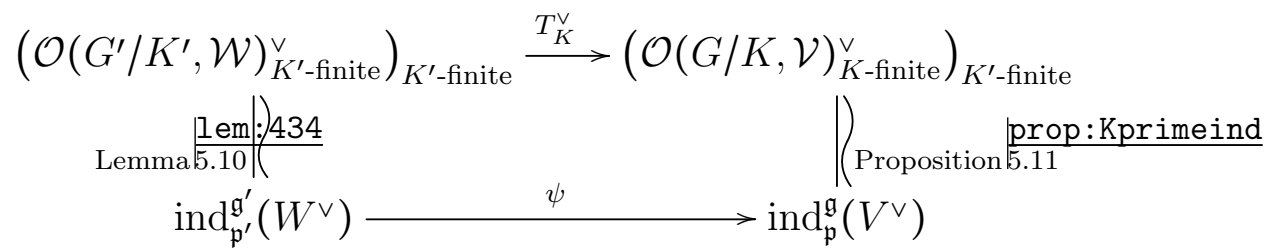

The correspondence $T_{K} \mapsto \psi$ is one-to-one, and thus we have obtained the following natural injective map

$$
\operatorname{Hom}_{\left(\mathfrak{g}^{\prime}, K^{\prime}\right)}\left(\mathcal{O}(G / K, \mathcal{V})_{K \text {-finite }}, \mathcal{O}\left(G^{\prime} / K^{\prime}, \mathcal{W}\right)_{K^{\prime} \text {-finite }}\right) \hookrightarrow \operatorname{Hom}_{\mathfrak{g}^{\prime}}\left(\operatorname{ind}_{\mathfrak{p}^{\prime}}^{\mathfrak{g}^{\prime}}\left(W^{\vee}\right), \operatorname{ind}_{\mathfrak{p}}^{\mathfrak{g}}\left(V^{\vee}\right)\right)
$$

According to Theorems 2.12 and 4.1 the latter space is isomorphic to Diff $G^{\prime}\left(\mathcal{V}_{X}, \mathcal{W}_{Y}\right)$. This shows that (5.4) is surjective.

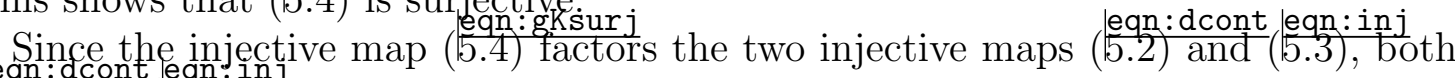
(5.2) and (5.3) are bijective.

5.3. Automatic continuity theorem in the unitary case. Any unitary highest weight module is realized as a subrepresentation of $\mathcal{O}(G / K, \mathcal{V})$ for some $G$ equivariant holomorphic vector bundle $\mathcal{V}$ over $G / K$. In this subsection, we prove that any continuous homomorphism between Fréchet modules $\mathcal{O}(G / K, \mathcal{V})$ and $\mathcal{O}\left(G^{\prime} / K^{\prime}, \mathcal{W}\right)$ induces a continuous homomorphism between their unitary submodules.

Definition 5.12. For a Fréchet $G$-module $\mathcal{F}$, we say a $G$-submodule $\mathcal{H}$ is a unitary submodule if $\mathcal{H}$ is a Hilbert space such that the inclusion map $\mathcal{H} \hookrightarrow \mathcal{F}$ is continuous and that $G$ acts unitarily on $\mathcal{H}$.

If $V$ is an irreducible $K$-module, then there exists at most one non-zero unitary submodule (up to a scaling of the inner product) of $\mathcal{O}(G / K, \mathcal{V})$. We denote by $\mathcal{H}_{V}^{G}$ the unitary submodule of $\mathcal{O}(G / K, \mathcal{V})$. The stassification of irreducible $K$-modules $V$ for which $\mathcal{H}_{V}^{G} \neq\{0\}$ was accomplished in EHWW83. We shall prove that any $G^{\prime}-$ equivariant differential operator in Theorem 5.3 preserves the unitary submodules in the following sense:

thm:CC Theorem 5.13. Let $G^{\prime}$ be a reductive subgroup of $G$ satisfying (5.1). Let $V$ and $W$ be any irreducible finite-dimensional representations of $K$ and $K^{\prime}$, respectively. Suppose that $T: \mathcal{O}(G / K, \mathcal{V}) \longrightarrow \mathcal{O}\left(G^{\prime} / K^{\prime}, \mathcal{W}\right)$ is a $G^{\prime}$-equivariant differential operator such that $\left.T\right|_{\mathcal{H}_{V}^{G}} \not \equiv 0$. Then $\mathcal{H}_{W}^{G^{\prime}} \neq\{0\}$ and $T$ induces a continuous $G^{\prime}$-equivariant linear map from the Hilbert space $\mathcal{H}_{V}^{G}$ onto the Hilbert space $\mathcal{H}_{W}^{G^{\prime}}$. 
Applying Theorems $\frac{t h m: C}{5.3}$ and $\frac{t h m \cdot C C}{5.13}$ to the setting of Example $\frac{b x \cdot c k k}{5.2}(1)$, we have:

Example 5.14. Any symmetry breaking operator for the tensor product of two holomorphic discrete series representations is given by a holomorphic differential operator if those representations are realized in the space of holomorphic sections for $G$-equivariant holomorphic vector bundles over the Hermitian symmetric space $G / K$. The Rankin-Cohen bidifferential operators are such operators for $G=S L(2, \mathbb{R})$.

Remark 5.15. As we shall see in the proof, the unitary representation $\mathcal{H}_{V}^{G}$ decomposes discretely when restricted to $G^{\prime}$ if the condition (5.1) is satisfied. The unitary submodule $\mathcal{H}_{W}^{G^{\prime}}$ occurs as a discrete summand of the restriction of the unitary representation $\mathcal{H}_{V}^{G}$ of $G$ to the subgroup $G^{\prime}$.

Let $V$ be an irreducible representation of $K$ as before. Then, there exists a unique $K$-submodule of $\mathcal{O}(G / K, \mathcal{V})_{K \text {-finite }} \simeq S\left(\mathfrak{n}_{+}\right) \otimes V$ isomorphic to $V$, namely $S^{0}\left(\mathfrak{n}_{+}\right) \otimes V \simeq$ $V$.

Lemma 5.16. Let $M$ be a non-zero $(\mathfrak{g}, K)$-submodule of $\mathcal{O}(G / K, \mathcal{V})_{K \text {-finite. Then, }}$

1) The module $M$ contains $V$.

2) If $M$ is unitarizable, then its Hilbert completion can be realized in $\mathcal{O}(G / K, \mathcal{V})$ and $M=\left(\mathcal{H}_{V}^{G}\right)_{K \text {-finite }}$.

Proof. 1) Since any non-zero quotient of the (generalized) Verma module ind $\mathrm{g}_{\mathfrak{p}}^{\mathfrak{g}}\left(V^{\vee}\right)$ contains $V^{\vee}$, the first statement follows from Lemma 5.10 . Alternatively, since the infinitesimal action of $\mathfrak{n}_{-}$on $\mathcal{O}(G / K, \mathcal{V})_{K \text {-finite }} \simeq \operatorname{Pol}\left(\mathfrak{n}_{-}\right) \otimes V$ is given by directional derivatives, iterated operators of $\mathfrak{n}_{-}$yield non-zero elements in $V$.

2) Denote by $(\pi, \bar{M})$ the unitary representation of $G$ obtained as an (abstract) Hilbert completion of the $(\mathfrak{g}, K)$-module $M$. We regard $V$ as a $K$-submodule of $M$, and also of $\bar{M}$. Then the map

$$
G \times \bar{M} \times V \longrightarrow \mathbb{C}, \quad(g, w, v) \mapsto(w, \pi(g) v)_{\bar{M}},
$$

induces an injective $G$-homomorphism $\iota: \bar{M} \longrightarrow \mathcal{O}(G / K, \mathcal{V})$. Since $\mathcal{H}_{V}^{G}$ is the unique non-zero unitary submodule, $\iota$ is an isomorphism onto $\mathcal{H}_{V}^{G}$.

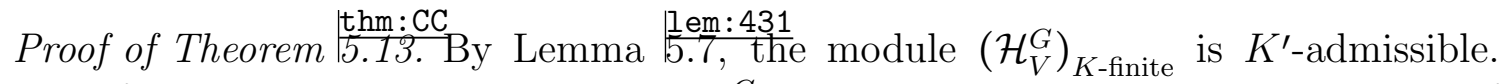
Therefore, the unitary representation $\mathcal{H}_{V}^{G}$ decomposes into a Hilbert direct sum of irreducible unitary representations $\left\{U_{j}\right\}$ of $G^{\prime}$ :

eqn : 431

$$
\mathcal{H}_{V}^{G} \simeq \sum_{j}^{\oplus} m_{j} U_{j}
$$

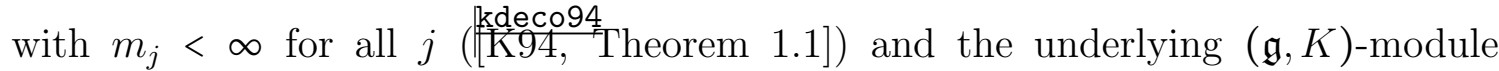
$\left(\mathcal{H}_{V}^{G}\right)_{K \text {-finite }}$ is isomorphic to an algebraic direct sum of irreducible and unitarizable 
$\left(\mathfrak{g}^{\prime}, K^{\prime}\right)$-modules

eqn : 432

$$
\left(\mathcal{H}_{V}^{G}\right)_{K \text {-finite }}=\left(\mathcal{H}_{V}^{G}\right)_{K^{\prime} \text {-finite }} \simeq \bigoplus_{j} m_{j}\left(U_{j}\right)_{K^{\prime} \text {-finite }},
$$

with the same multiplicities $\frac{\pi \operatorname{dec} 098}{\text { K98 }}$. (We remark that an analogous statement fails for the restriction $\left.\pi\right|_{G^{\prime}}$ of an irreducible unitary representation $\pi$ of $G$ if the branching law contains continuous spectrum).

As we saw in the proof of Theorem 5.3 , the $G^{\prime}$-equivariant differential operators $T$ induces a $\left(\mathfrak{g}^{\prime}, K^{\prime}\right)$-homomorphism

$$
T_{K}:\left(\mathcal{H}_{V}^{G}\right)_{K \text {-finite }} \longrightarrow \mathcal{O}\left(G^{\prime} / K^{\prime}, \mathcal{W}\right)_{K^{\prime} \text {-finite }}
$$

By $\frac{132}{5.7}, M:=T_{K}\left(\left(\mathcal{H}_{V}^{G}\right)_{K \text {-finite }}\right)$ is an algebraic direct sum of some irreducible unitarizable $\left(\mathfrak{g}^{\prime}, K^{\prime}\right)$-modules. Since $\mathcal{O}\left(G^{\prime} / K^{\prime}, \mathcal{W}\right)_{K^{\prime} \text {-finite }}$ contains at most one irreducible unitarizable $\left(\mathfrak{g}^{\prime}, K^{\prime}\right)$-module, $M$ is irreducible as a $\left(\mathfrak{g}^{\prime}, K^{\prime}\right)$-module, and we can realize its Hilbert completion $\operatorname{as}_{3} \mathcal{Z}_{W}^{G^{\prime}}$ by Lemma

In view of 5.6 and $(5.7)$, there exists a continuous $G^{\prime}$-homomorphism between Hilbert spaces:

$$
\widetilde{T}: \mathcal{H}_{V}^{G} \longrightarrow \mathcal{H}_{W}^{G^{\prime}}
$$

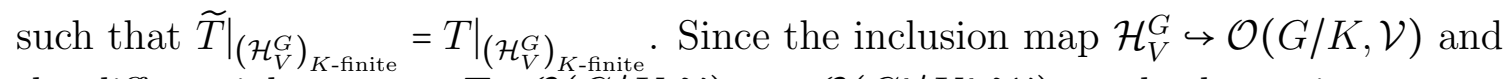
the differential operator $T: \mathcal{O}(G / K, \mathcal{V}) \longrightarrow \mathcal{O}\left(G^{\prime} / K^{\prime}, \mathcal{W}\right)$ are both continuous, we get $\widetilde{T}=T$ on $\mathcal{H}_{V}^{G}$. Hence Theorem is proved.

5.4. Orthogonal projectors. If $Y$ is one-dimensional and $\left(G, G_{0.0}^{\prime}\right.$ is a reductive symmetricopair satisfying (5.1), then all the multiplicities $m_{j}$ in (5.6) are equal to one (see [K08]) and it becomes meaningful to describe the projector from $\mathcal{H}_{V}^{G}$ to each $G^{\prime}$-irreducible summand. We explain briefly the relationship between the projector for the unitary representation and the symmetry breaking operator.

For this, suppose $T: \mathcal{O}(G / K, \mathcal{V}) \rightarrow O\left(G^{\prime} / K^{\prime}\right.$. $\left.\mathcal{L}\right)$ is a $G^{\prime}$-equivariant differential operator such that $\left.T\right|_{\mathcal{H}_{V}^{G}} \neq \equiv$. By Theorem $5.13 . T$ induces a continuous map $T$ : $\mathcal{H}_{V}^{G} \rightarrow \mathcal{H}_{W}^{G^{\prime}}$. Let $T^{*}: \mathcal{H}_{W}^{G^{\prime}} \rightarrow \mathcal{H}_{V}^{G}$ be its the adjoint operator. Then the composition $T^{\star} T: \mathcal{H}_{V}^{G} \rightarrow \mathcal{H}_{V}^{G}$ is a $G^{\prime}$-intertwining operator onto the $G^{\prime}$-irreducible summand which is isomorphic to $\mathcal{H}_{W}^{G^{\prime}}$. Since $T$ vanishes on the orthogonal complement to $T^{\star}\left(\mathcal{H}_{W}^{G^{\prime}}\right)$, it is (up to scaling) the orthogonal projector onto $\mathcal{H}_{W}^{G^{\prime}}$.

Explicit description of such differential operators $T$ will be the main concern of the second part [KP15-2] of this work.

Acknowledgements. T. Kobayashi was partially supported by Institut des Hautes Études Scientifiques, France and Grant-in-Aid for Scientific Research (B) (22340026) and (A) (25247006), Japan Society for the Promotion of Science. Both authors were 
partially supported by Max Planck Institute for Mathematics (Bonn) where a large part of this work was done.

\section{REFERENCES}

[DP07] G. van Dijk, M. Pevzner, Ring structures for holomorphic discrete series and Rankin-Cohen brackets. J. Lie Theory, 17, (2007), pp. 283-305.

[EHW83] Th. Enright, R. Howe, N. Wallach, A classification of unitary highest weight modules. Representation theory of reductive groups (Park City, Utah, 1982), pp. 97-143, Progress in Mathematics, 40, Birkhäuser, Boston, MA, 1983.

[HJ82] M. Harris, H. P. Jakobsen, Singular holomorphic representations and singular modular forms. Math. Ann. 259 (1982), pp.227-244.

[J09] A. Juhl, Families of conformally covariant differential operators, Q-curvature and holography. Progress in Mathematics, 275. Birkhäuser, Basel, 2009.

[KnSt71] A. W. Knapp, E. M. Stein, Intertwining operators for semisimple groups. Ann. of Math. 93 (1971), pp. 489-578

[K94] T. Kobayashi, Discrete decomposability of the restriction of $A_{\mathfrak{q}}(\lambda)$ with respect to reductive subgroups and its applications, Invent. Math. 117 (1994), pp. 181-205.

[K98a] T. Kobayashi, Discrete decomposability of the restriction of $A_{\mathfrak{q}}(\lambda)$ with respect to reductive subgroups II-micro-local analysis and asymptotic K-support, Ann. of Math. (2) 147 (1998), pp. 709-729.

[K98b] T. Kobayashi, Discrete decomposability of the restriction of $A_{\mathfrak{q}}(\lambda)$ with respect to reductive subgroups III-restriction of Harish-Chandra modules and associated varieties, Invent. Math. 131 (1998), pp. 229-256.

[K08] T. Kobayashi, Multiplicity-free theorems of the restrictions of unitary highest weight modules with respect to reductive symmetric pairs. Representation theory and automorphic forms, Progr. Math., 255, Birkhäuser, Boston, (2008), pp. 45-109

[K12] T. Kobayashi, Restrictions of generalized Verma modules to symmetric pairs, Transformation Groups 17, (2012), pp. 523-546.

[K13] T. Kobayashi. F-method for constructing equivariant differential operators. Contemporary Mathematics,598 (2013), pp. 141-148. Amer. Math. Soc.

[K14] T. Kobayashi, F-method for symmetry breaking operators, Diff. Geometry and its Appl. 33, (2014), pp. 272-289.

[KØSS13] T. Kobayashi, B. Ørsted, P. Somberg, V. Souček, Branching laws for Verma modules and applications in parabolic geometry. Part I. Preprint. ArXiv:1305.6040.

[KKP15] T. Kobayashi, T. Kubo, M. Pevzner, Vector-valued covariant differential operators for the Möbius transformation. In Lie theory and its applications in Physics. V. Dobrev (Ed.) Springer Proceedings in Mathematics and Statistics. 111 (2015) pp. 67-86.

[KP15-2] T. Kobayashi, M. Pevzner, Differential symmetry breaking operators. II. RankinCohen operators for symmetric pairs, to appear in Selecta Mathematica (the same issue), arXiv:1301.2111.

[KS14] T. Kobayashi, B. Speh, Intertwining operators and the restriction of representations of rank one orthogonal groups, C. R. Acad. Sci. Paris, Sér. I. 352, (2014), pp. 89-94.

[Kos74] B. Kostant, Verma modules and the existence of quasi-invariant differential operators, Lecture Notes in Math. 466, Springer-Verlag, (1974), pp. 101-129.

[Pee59] J. Peetre, Une caractérisation abstraite des opérateurs différentiels, Math. Scand. 7 (1959), pp. 211-218.

[S66] L. Schwartz, Théorie des distributions. Hermann, Paris, 1966.

T. Kobayashi. Kavli IPMU and Graduate School of Mathematical Sciences, The University of Tokyo, 3-8-1 Komaba, Meguro, Tokyo, 153-8914 Japan; toshi@ms.u-tokyo.ac.jp. 
M. Pevzner. Laboratoire de Mathématiques de Reims, Université de Reims-Champagne-Ardenne, FR 3399 CNRS, F-51687, Reims, France; pevzner@univ-reims.fr. 